Conjugação de involuções e suas aplicações 

SERVIÇO DE PÓS-GRADUAÇÃO DO ICMC-USP

Data de Depósito:

Assinatura:

\title{
Conjugação de involuções e suas aplicações
}

\author{
Elizabeth Ruth Salazar Flores
}

Orientadora: Profa. Dra. Miriam Garcia Manoel

Dissertação apresentada ao Instituto de Ciências Matemáticas e de Computação - ICMC-USP, como parte dos requisitos para obtenção do título de Mestre em Ciências - Matemática . VERSÃO REVISADA

USP - São Carlos

Julho de 2013 
Ficha catalográfica elaborada pela Biblioteca Prof. Achille Bassi e Seção Técnica de Informática, ICMC/USP, com os dados fornecidos pelo(a) autor(a)

\begin{tabular}{|c|c|}
\hline \multirow[t]{3}{*}{ S159C } & $\begin{array}{l}\text { Salazar Flores, Elizabeth Ruth } \\
\text { Conjugação de involuções e suas aplicações / } \\
\text { Elizabeth Ruth Salazar Flores; orientadora Miriam } \\
\text { Garcia Manoel. -- São Carlos, } 2013 . \\
\quad 74 \text { p. }\end{array}$ \\
\hline & $\begin{array}{l}\text { Dissertação (Mestrado - Programa de Pós-Graduação } \\
\text { em Matemática) -- Instituto de Ciências Matemáticas } \\
\text { e de Computação, Universidade de São Paulo, } 2013 .\end{array}$ \\
\hline & $\begin{array}{l}\text { 1. Involuções. 2. Dobras. 3. Formas normais de } \\
\text { pares de involuções. 4. Difeomorfismos normalmente } \\
\text { hiperbólicos. 5. Grupos gerados por pares de } \\
\text { involuções. I. Garcia Manoel, Miriam, orient. II. } \\
\text { Título. }\end{array}$ \\
\hline
\end{tabular}


Aos meus pais!!! 



\section{Agradecimentos}

Primeiramente agradeço a Deus, por ser meu refúgio e por ter dado a mim forças para alcançar meus objetivos.

Aos meus amados pais, por ter acreditado em mim e pelo apoio incondicional. Aos meus irmãos Raúl, Augusto, Miguel Angel, e às minhas irmãs Coty e Haydeé sou grata por muitos motivos.

À minha orientadora, Miriam Manoel, pelo tema proposto e por ter aceitado me orientar sem me conhecer. A Solange, pela ajuda e o tempo neste trabalho.

Aos meus caros colegas e amigos, Jorge Curipaco, Lito, Rosita, Jaqui, Pathy, Norbil, Iris e Renato , sou grata pela amizade, companhia e pela ajuda que, deram sempre que precisei. A Claudio, pelo apoio e pela motivação para começar o mestrado.

À Edison, por ter estado ao meu lado nesses últimos tempos, me apoiando e compreendendo, assim como pela sua paciência, carinho e atenção.

À CNPq pelo suporte finaceiro concedido durante o mestrado.

Enfim, à todos aqueles que colaboraram de alguma forma para a realização desse trabalho. 


\section{Resumo}

Este trabalho propõe direcionar o estudo de involuções para dois ramos de pesquisa dentro da teoria das Singularidades e de Sistemas Dinâmicos. Mais precisamente, tratamos sua interligação com diagramas divergentes de dobras e seu aparecimento nos sistemas dinâmicos discretos reversíveis. No primeiro, tratamos da importante relação entre a classificação de diagramas divergentes de dobras, digamos de $s$ dobras, e a classificação de $s$-uplas de involuções associadas a estes diagramas. No segundo contexto, o estudo se volta para a questão sobre condições para a linearização simultânea de uma classe de pares de involuções e a obtenção de formas normais desses pares. 


\section{Abstract}

This work proposes to address the study of involutions for two branches of research into the theory of Singularities and Dynamical Systems. More precisely, we treat its interconnection with divergent diagrams of folds and their appearance in discrete reversible dynamical systems. First, we treat the important relationship between the classification of divergent diagrams of folds, say $s$ folds, and the classification of $s$-tuples of involutions associated with these diagrams. In the second context, the study turns to the question of conditions for simultaneous linearization of a class of pairs of involutions and the deduction of the normal forms of these pairs. 


\section{Sumário}

1 Preliminares 1

1.1 Dobras e involuções . . . . . . . . . . . . . . . . . . . 1

1.2 Diagramas divergentes de dobras . . . . . . . . . . . . . . . 6

1.3 Conjuntos de involuções transversais . . . . . . . . . . . . 7

1.3.1 Formas normais de pares de involuções transversais com dimensão do subespaço de pontos fixos arbitrária . . . . . . . . . 8

1.3.2 Involuções $\varphi$ com $\operatorname{codim} \mathcal{F}(\varphi)=1 \ldots \ldots \ldots . \ldots \ldots$

2 Formas normais de pares de involuções e diagramas divergentes de $\begin{array}{ll}\text { dobras } & \mathbf{1 5}\end{array}$

2.1 Caracterização de órbitas . . . . . . . . . . . . . . . . . 15

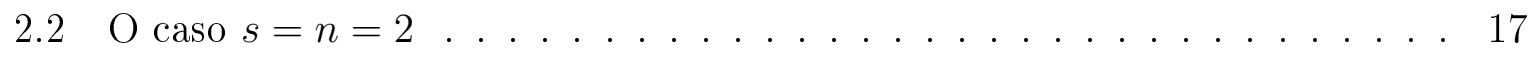

2.2.1 Formas normais de pares de involuções . . . . . . . . . . . 17

2.2.2 Formas normais de diagramas divergentes de dobras . . . . . . 20

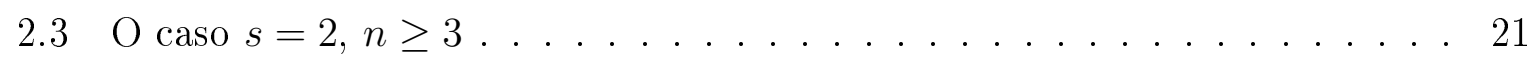

2.3.1 Formas normais de pares de involuções . . . . . . . . . . . . . 22

2.3.2 Formas normais de diagramas divergentes de dobras . . . . . . . . 28

3 Diagramas divergentes de dobras, pares de involuções e sistemas dinâmicos

3.1 Campos vetoriais descontínuos . . . . . . . . . . . . . 31

3.2 Difeomorfismos reversíveis . . . . . . . . . . . . . . 33

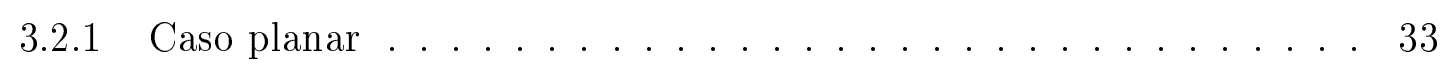

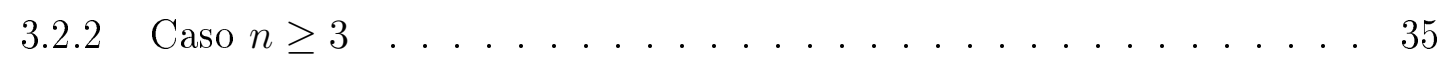

4 Grupos gerados por um par de involuções e a teoria reversível equivariante 
4.1 Grupos gerados por um par de involuções . . . . . . . . . . . . . . . . 40

$4.1 .1 \quad$ Caso $n=2 \ldots \ldots \ldots \ldots \ldots$

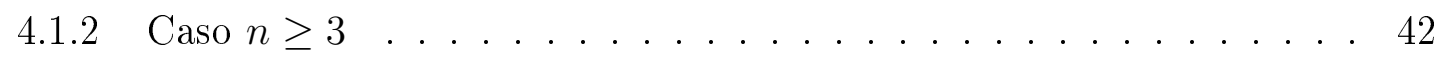

4.2 A teoria reversível equivariante . . . . . . . . . . . . . 44

4.2 .1 Teoria de grupos . . . . . . . . . . . . . . . . . 45

4.2.2 Germes de funções invariantes e de aplicações equivariantes . . . . . 45

4.3 Grupos conjugados e a teoria reversível equivariante . . . . . . . . . . . . 47

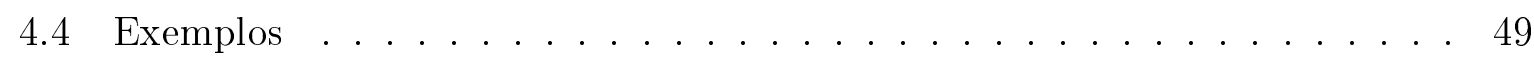

$5 \quad$ Pares de involuções com composta normalmente hiperbólica 53

5.1 Um teorema de linearização . . . . . . . . . . . . . . . 53

5.2 Classificação de pares de involuções lineares transversais com composta normalmente hiperbólica . . . . . . . . . . . . . 56

5.2 .1 Primeiros resultados sobre hiperbolicidade . . . . . . . . . 56

5.2 .2 A classificação . . . . . . . . . . . . . . . . . 57

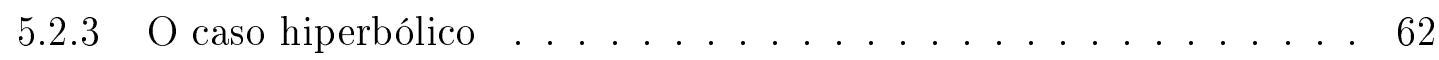

5.2 .4 O caso geral . . . . . . . . . . . . . 65

$\begin{array}{ll}\text { Referências Bibliográficas } & 73\end{array}$ 


\section{Notação}

$\mathcal{F}(\varphi)$ : Subespaço de pontos fixos de uma involução $\varphi ;$

$\Sigma(f)$ : Conjunto dos pontos singulares de uma aplicação $f$;

$\mathcal{A}(\varphi)$ : Subespaço antipodal de uma aplicação $\varphi ;$

$\operatorname{tr}(\varphi)$ : Traço de uma aplicação $\varphi$;

$R_{\theta}:$ Rotação de ângulo $\theta$;

$D_{m}:$ Grupo diedral de ordem $m$;

$\mathcal{P}_{V}(\Gamma):$ Anel das funções polinomiais $f: V \rightarrow \mathbb{R} \Gamma$-invariantes;

$\mathcal{E}_{V}(\Gamma)$ : Anel dos germes de funções $f:(V, 0) \rightarrow \mathbb{R} \Gamma$-invariantes;

$\mathcal{Q}_{V}(\Gamma)$ : Módulo sobre $\mathcal{P}_{V}(\Gamma)$ das funções polinomiais $f: V \rightarrow \mathbb{R} \Gamma$-anti-invariantes;

$\mathcal{F}_{V}(\Gamma)$ : Módulo sobre $\mathcal{E}_{V}(\Gamma)$ dos germes de funções $f:(V, 0) \rightarrow \mathbb{R} \Gamma$-anti-invariantes;

$\overrightarrow{\mathcal{P}}_{V, W}(\Gamma)$ : Módulo sobre $\mathcal{P}_{V}(\Gamma)$ das aplicações polinomiais $g: V \rightarrow W \Gamma$-equivariantes;

$\overrightarrow{\mathcal{E}}_{V, W}(\Gamma)$ : Módulo sobre $\mathcal{E}_{V}(\Gamma)$ dos germes de aplicações $g:(V, 0) \rightarrow W \Gamma$-equivariantes;

$\overrightarrow{\mathcal{Q}}_{V, W}(\Gamma)$ : Módulo sobre $\mathcal{P}_{V}(\Gamma)$ das aplicações polinomiais $g: V \rightarrow W \Gamma$-reversíveisequivariantes;

$\overrightarrow{\mathcal{F}}_{V, W}(\Gamma)$ : Módulo sobre $\mathcal{E}_{V}(\Gamma)$ dos germes de aplicações $g:(V, 0) \rightarrow W \Gamma$-reversíveisequivariantes; 


\section{Introdução}

O estudo desenvolvido neste trabalho é de natureza local, com particular interesse em alguns ramos da teoria de singularidades e dos sistemas dinâmicos. As involuções têm sido objeto de interesse de diversos autores sob diferentes aspectos. Seu tratamento neste projeto é enfatizado com respeito à sua associação com diagramas divergentes de dobras e com os sistemas dinâmicos discretos reversíveis.

O estudo proposto aborda a classificação de $s$-uplas de involuções segundo a relação de equivalência dada por conjugação simultânea das involuções correspondentes, ou seja, duas $s$-uplas $\left(\varphi_{1}, \ldots, \varphi_{s}\right)$ e $\left(\psi_{1}, \ldots, \psi_{s}\right)$ de involuções em $\left(\mathbb{R}^{n}, 0\right)$ são equivalentes se existe um germe de difeomorfismo $h$ de $\left(\mathbb{R}^{n}, 0\right)$ tal que

$$
\psi_{i}=h \circ \varphi_{i} \circ h^{-1}, i=1, \ldots, s .
$$

Preliminarmente, são estudadas em detalhes as definições e propriedades básicas do assunto. Uma involução é um germe de difeomorfismo $\varphi:\left(\mathbb{R}^{n}, 0\right) \rightarrow\left(\mathbb{R}^{n}, 0\right)$ satisfazendo $\varphi \circ \varphi=\mathrm{I}$, onde I denota o germe da identidade em $\left(\mathbb{R}^{n}, 0\right)$. Tem um papel crucial aqui o conjunto de pontos fixos de $\varphi$, dado por

$$
\mathcal{F}(\varphi)=\left\{x \in\left(\mathbb{R}^{n}, 0\right): \varphi(x)=x\right\}
$$

Ambos os contextos em que abordamos conjuntos de involuções neste trabalho assumen a condição de transversalidade: um conjunto $\left\{\varphi_{1}, \ldots, \varphi_{s}\right\}$ de involuções em $\left(\mathbb{R}^{n}, 0\right)$, $s \leq n$, é chamado transversal se $\mathcal{F}\left(\varphi_{i}\right)$ é transversal a $\mathcal{F}\left(\varphi_{j}\right)$ em 0 para $i \neq j$ e $\operatorname{codim} \cap_{i=1}^{s} T_{0} \mathcal{F}\left(\varphi_{i}\right)=\sum_{1}^{s} \operatorname{codim} \mathcal{F}\left(\varphi_{i}\right)$, onde $T_{0} \mathcal{F}\left(\varphi_{i}\right)$ denota o espaço tangente a $\mathcal{F}\left(\varphi_{i}\right)$ na origem.

A primeira parte de nosso estudo tem como referência [10] e trata da relação entre a classificação de diagramas divergentes de dobras $\left(f_{1}, \ldots, f_{s}\right)$ e a classificação de $s$-uplas de involuções $\left(\varphi_{1}, \ldots, \varphi_{s}\right)$ com $\varphi_{i} \neq \mathrm{I}$ associadas a estes diagramas, isto é, $f_{i} \circ \varphi_{i}=f_{i}$ para $i=1, \ldots, s$. O principal resultado em [10] é uma relação um-a-um entre a classificação de $s$-uplas de involuções e os diagramas divergentes de dobras associados a estas. 
Uma ênfase é dada para a classificação quando $s=2$ e sob a condição de transversalidade dos pares de involuções. Para a classificação destes pares precisamos informação da estrutura do grupo $\Delta=\left\langle\varphi_{1}, \varphi_{2}\right\rangle$, isto é, conhecer se o grupo $\Delta$ é Abeliano ou não. Esta classificação permite encontrar uma invariante para estas classes de diagramas divergentes de dobras a qual é o traço da parte linear da composta $\varphi_{1} \circ \varphi_{s}$, essa invariante tem um papel importante dado que a maioria de formas normais de pares de involuções transversais são caracterizados por esta.

Adicionamos a interação entre campos vetoriais descontínuos e diagramas divergentes de dobras e se aplica o estudo da classificação feito aos sistemas discretos reversíveis.

Notemos que se o grupo $\Delta=\left\langle\varphi_{1}, \varphi_{2}\right\rangle$ é Abeliano, então é do tipo $\mathbb{Z}_{2} \times \mathbb{Z}_{2}$. Assim, aparece o interesse de identificar a estrutura algébrica dos grupos gerados por pares de involuções. Depois de fazer esta identificação, introduzimos a teoria reversível equivariante onde usamos como referência [13] e aplicamos a estes grupos encontrados.

A segunda parte deste estudo envolve a hiperbolicidade normal da composta $\varphi_{1} \circ \varphi_{2}$ para o par $\left(\varphi_{1}, \varphi_{2}\right)$ de involuções e tem como referência básica [11]. Dado que a linearização de $\varphi_{1}$ e $\varphi_{2}$ é uma condição suficiente para a linearização de $\varphi_{1} \circ \varphi_{2}$, surge a questão sobre a linearização do par $\left(\varphi_{1}, \varphi_{2}\right)$. Em [11] os autores resolvem esta questão para pares cuja composta é normalmente hiperbólica, esse resultado é mencionado no Teorema 5.2. A definição é a seguinte: Seja $f:\left(\mathbb{R}^{n}, 0\right) \rightarrow\left(\mathbb{R}^{n}, 0\right)$ um germe de difeomorfismo, $f \neq \mathrm{I}$. Suponha $\mathcal{F}(f)$ uma subvariedade de $\left(\mathbb{R}^{n}, 0\right)$ e que $\operatorname{dim} \mathcal{F}(f)=k$. $f$ é chamado normalmente hiperbólico se o espectro de $d f(0)$ tem, contando multiplicidade, $n-k$ elementos fora do círculo unitário $S^{1} \cap \mathbb{C}$. Estudamos a classificação para certos pares de involuções lineares incluindo o caso particular em que a composta $\varphi_{1} \circ \varphi_{2}$ é hiperbólica e notamos que se duas involuções têm composta normalmente hiperbólica, então o grupo gerado por estas involuções não é Abeliano.

Este texto é organizado da seguinte forma: nos Capítulos 1 e 2 apresentamos a classificação das formas normais de pares de involuções e as correspondentes formas normais de diagramas divergentes de dobras associados. No Capítulo 3 apresentamos a interação entre campos vetoriais descontínuos e diagramas divergentes de dobras e o estudo de sistemas dinâmicos reversíveis usando os resultados de classificação obtidos no Capítulo 2 para o caso planar e logo apresentamos uma generalização ao caso $n$-dimensional. A referência básica do estudo aprensentado nestes capítulos são [10].

No Capítulo 4 damos a descrição dos grupos gerados por pares de involuções lineares 
transversais primeiramente em dimensão 2 e depois em dimensões maiores iguais que 3. Fazemos também aqui um estudo sobre a base da teoria reversível equivariante o aplicamos a estes grupos de involuções .

No Capítulo 5 mencionamos um teorema de linearização para pares de involuções com composta normalmente hiperbólica. Na Seção 5.2 apresentamos a classificação dos pares lineares via equivalencia linear e apresentamos uma caracterização do conjunto de pares com composta hiperbólica dentro da classe dos normalmente hiperbólicos. Adicionalmente, explicitamos a classificação para pares en dimensões específicas. Como último resultado desta seção, apresentamos a relação entre as subvariedades $\mathcal{F}\left(\varphi_{1}\right), \mathcal{F}\left(\varphi_{2}\right)$ e $\mathcal{F}\left(\varphi_{1} \circ \varphi_{2}\right)$ para quaisquer pares de involuções com composta normalmente hiperbólica. 


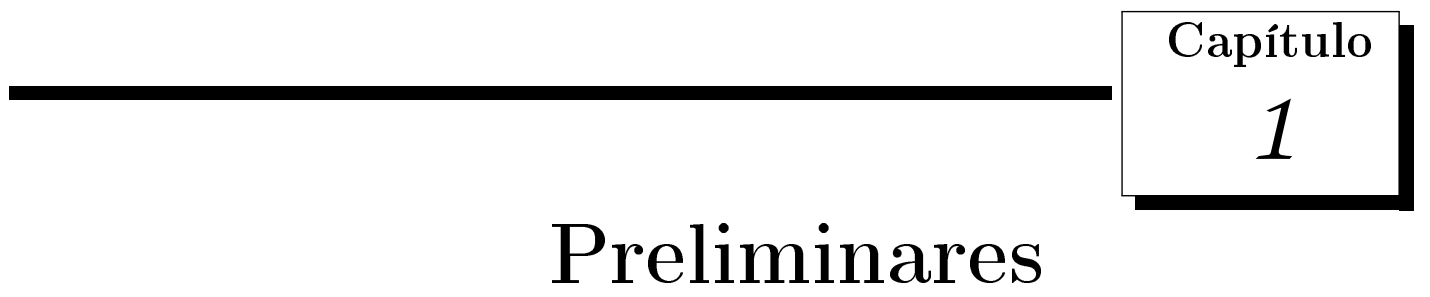

Neste capítulo apresentamos os objetos principais desta dissertação, que são involuções e dobras. Apresentamos também a definição de associatividade entre elas e os resultados de classificação de diagramas divergentes de dobras. Como veremos, tal classificação está intimamente ligada à classificação das $s$-uplas de involuções associadas. Nossa referência básica é [10].

Definimos o conjunto dos pontos fixos de uma involução $\varphi$, denotado por $\mathcal{F}(\varphi)$, e introduzimos a definição de tranversalidade de involuções. Descrevemos as formas prenormais de pares de involuções lineares transversais com codim $\mathcal{F}(\varphi)$ arbitrária e uma condição necessária e suficiente para que dois pares de involuções deste tipo sejam equivalentes.

Apresentamos as formas prenormais de uma $s$-upla de involuções lineares transversais e assim descrevemos as formas prenormais dos diagramas divergentes de dobras associados a estas $s$-uplas.

Finalmente, apresentamos explicitamente a forma normal de uma $s$-upla de involuções transversais não necessariamente lineares tais que o grupo gerado por estas involuções é Abeliano. Em conseqüência, obtemos também as formas normais de diagramas divergentes de dobras associados a esta $s$-upla de involuções.

\subsection{Dobras e involuções}

Os conceitos de dobras e involuções tratados aqui são de natureza local e damos suas definições usando a noção de germe, definição básica da teoria de singularidades.

Definição 1.1: Uma involução é um germe de difeomorfismo $\varphi:\left(\mathbb{R}^{n}, 0\right) \rightarrow\left(\mathbb{R}^{n}, 0\right)$ 
satisfazendo $\varphi \circ \varphi=\mathrm{I}$.

Vamos denotar por $\mathcal{F}(\varphi)$ o conjunto de pontos fixos de $\varphi$, isto é,

$$
\mathcal{F}(\varphi)=\left\{x \in\left(\mathbb{R}^{n}, 0\right): \varphi(x)=x\right\}
$$

Definição 1.2: Duas $s$-uplas $\left(\varphi_{1} \ldots \varphi_{s}\right)$ e $\left(\psi_{1} \ldots \psi_{s}\right)$ de involuções em $\left(\mathbb{R}^{n}, 0\right)$ são equivalentes se existe um germe de difeomorfismo $h$ de $\left(\mathbb{R}^{n}, 0\right)$ tal que $\psi_{i}=h \circ \varphi_{i} \circ h^{-1}$, para todo $i=1, \ldots, s$.

O germe de difeomorfismo $h$ da Definição 1.2 é nada mais que uma conjugação simultânea das involuções correspondentes das $s$-uplas . Notemos que este $h$ satisfaz

$$
h\left(\mathcal{F}\left(\varphi_{i}\right)\right)=\mathcal{F}\left(\psi_{i}\right), i=1, \ldots, s
$$

De fato, se $x \in \mathcal{F}\left(\psi_{i}\right)$ temos que, $\psi_{i}(x)=x=h \circ \varphi_{i}\left(h^{-1}(x)\right)$ o que implica que $h^{-1}(x) \in$ $\mathcal{F}\left(\varphi_{i}\right)$ e, portanto, $x \in h\left(\mathcal{F}\left(\varphi_{i}\right)\right)$. Se $x \in \mathcal{F}\left(\varphi_{i}\right)$, temos que $\psi_{i}(h(x))=h\left(\varphi_{i}(x)\right)=h(x)$ e, portanto, $h(x) \in \mathcal{F}\left(\psi_{i}\right)$.

Consideremos o conjunto $G_{s}=\left\{\varphi_{1} \ldots \varphi_{s}\right\}$ de involuções em $\left(\mathbb{R}^{n}, 0\right)$ e $\Delta_{s}=\left\langle\varphi_{1} \ldots \varphi_{s}\right\rangle$ o grupo gerado por estas involuções. O seguinte resultado segue do Teorema de BochnerMontgomery em [12]:

Teorema 1.3: Se $\Delta_{s}$ é um grupo Abeliano, então a s-upla $\left(\varphi_{1} \ldots \varphi_{s}\right)$ de involuções é equivalente a uma s-upla de involuções lineares.

Do teorema anterior temos que qualquer involução $\varphi:\left(\mathbb{R}^{n}, 0\right) \rightarrow\left(\mathbb{R}^{n}, 0\right)$ é conjugada a uma involução linear. Para uma única involução podemos, alternativamente, explicitar um germe de difeomorfismo que conjuga $\varphi$ e sua diferencial $d \varphi(0)$ na origem:

$$
h=\frac{1}{2}(\mathrm{I}+d \varphi(0) \circ \varphi) .
$$

Segue-se de $(1.1)$ que $\mathcal{F}(\varphi)$ é localmente difeomorfo a um subespaço linear de $\mathbb{R}^{n}$. Portanto, $\mathcal{F}(\varphi)$ é uma subvariedade em $\left(\mathbb{R}^{n}, 0\right)$. Desta forma, podemos caracterizar uma involução $\varphi$ a través da dimensão de $\mathcal{F}(\varphi)$ :

1. se $\operatorname{codim} \mathcal{F}(\varphi)=0$. Então, $\varphi$ é a identidade I;

2. se $\operatorname{codim} \mathcal{F}(\varphi)=l \neq 0$, então $\varphi$ é conjugada à forma canônica

$$
\left(x_{1}, \ldots, x_{n}\right) \mapsto\left(-x_{1}, \ldots,-x_{l}, x_{l+1}, \ldots, x_{n}\right)
$$


Outro conceito básico para os capítulos seguintes é:

Definição 1.4: Um germe $f:\left(\mathbb{R}^{n}, 0\right) \rightarrow\left(\mathbb{R}^{n}, 0\right)$ é uma dobra se existem germes de difeomorfismos $h$ e $k$ de $\left(\mathbb{R}^{n}, 0\right)$ tal que $f=k \circ f^{0} \circ h^{-1}$, onde

$$
f^{0}\left(x_{1}, \ldots, x_{n}\right)=\left(x_{1}^{2}, x_{2} \ldots, x_{n}\right) .
$$

Agora apresentamos a definição que relaciona uma dobra com uma involução:

Definição 1.5: Dada uma involução $\varphi$ em $\left(\mathbb{R}^{n}, 0\right)$ e uma dobra $f:\left(\mathbb{R}^{n}, 0\right) \mapsto\left(\mathbb{R}^{n}, 0\right)$ dizemos que $f$ é associado a $\varphi$, ou $\varphi$ é associado a $f$, se $\varphi \neq$ I e $f \circ \varphi=f$.

A seguir apresentamos alguns resultados relativos a uma involução e à dobra associada a esta.

Proposição 1.6: Dada uma dobra $f:\left(\mathbb{R}^{n}, 0\right) \mapsto\left(\mathbb{R}^{n}, 0\right)$, existe uma única involução associada a $f$.

Demonstração: Seja $f^{0}$ a dobra tal que $f^{0}\left(x_{1}, \ldots, x_{n}\right)=\left(x_{1}^{2}, x_{2}, \ldots, x_{n}\right)$. É fácil ver que $f^{0}$ esta associada à involução $\varphi^{0}\left(x_{1}, \ldots, x_{n}\right)=\left(-x_{1}, \ldots, x_{n}\right)$. Seja $\varphi$ outra involução associada a $f^{0}$, onde $\varphi=\left(\varphi_{1}, \ldots, \varphi_{s}\right)$, isto é,

$$
f^{0}=\left(x_{1}^{2}, x_{2}, \ldots, x_{n}\right)=f^{0} \circ \varphi=f^{0}\left(\varphi_{1}, \varphi_{2}, \ldots, \varphi_{n}\right)=\left(\varphi_{1}^{2}, \varphi_{2}, \ldots, \varphi_{n}\right) .
$$

Disto temos $\varphi_{1}^{2}=x_{1}^{2}$ e $\varphi_{i}=x_{i}$ para $i=2, \ldots, n$. Como $\varphi \neq$ I temos que $\varphi_{1}=-x_{1}$ e, portanto, $\varphi=\varphi^{0}$. Agora, seja $f$ uma dobra qualquer. Pela definição, sabemos que existem $k$ e $h$ germes de difeomorfismos tal que $f=k \circ f^{0} \circ h^{-1}$. Definindo a involução $\varphi$ por

$$
\varphi=h \circ \varphi^{0} \circ h^{-1}
$$

observamos que $f$ é associada a $\varphi$, isto é, $f \circ \varphi=f$. Seja $\psi$ outra involução. Então $f \circ \varphi=f \circ \psi=f$. Logo,

$$
\begin{aligned}
f^{0} \circ h^{-1} \circ \psi \circ h & =k^{-1} \circ k \circ f^{0} \circ h^{-1} \circ \psi \circ h \\
& =k^{-1} \circ f \circ \psi \circ h \\
& =k^{-1} \circ f \circ h \\
& =f^{0},
\end{aligned}
$$

o que significa que $h^{-1} \circ \psi \circ h$ é uma involução associada a $f^{0}$. Pela unicidade de $\varphi^{0}$, temos que $h^{-1} \circ \psi \circ h=\varphi^{0}$ e, portanto, $\psi=h \circ \varphi^{0} \circ h^{-1}=\varphi$. 
Para a involução $\varphi^{0}$ dada na prova da Proposição 1.6 temos $\operatorname{codim} \mathcal{F}\left(\varphi^{0}\right)=1$. Segue, então, que para qualquer dobra $f$ associada $\varphi$, temos

$$
\operatorname{codim} \mathcal{F}(\varphi)=\operatorname{codim} \mathcal{F}\left(\varphi^{0}\right)=1,
$$

sendo $\varphi$ e $\varphi^{0}$ conjugadas. Além disso, notemos que

$$
\mathcal{F}(\varphi)=\Sigma(f)
$$

onde $\Sigma(f)$ denota o conjunto dos pontos singulares de $f$. De fato, notemos que $\mathcal{F}\left(\varphi^{0}\right)=$ $\left\{\left(0, x_{2}, \ldots, x_{n}\right) \in\left(\mathbb{R}^{n}, 0\right)\right\}$

$$
\left(d f^{0}\right)\left(x_{1}, \ldots, x_{n}\right)=\left(\begin{array}{rrrr}
2 x_{1} & 0 & \ldots & 0 \\
0 & 1 & & \vdots \\
\vdots & & \ddots & 0 \\
0 & \ldots & 0 & 1
\end{array}\right) .
$$

Logo $\left(x_{1}, \ldots, x_{n}\right)$ é ponto singular de $f$ se, e somente se, $x_{1}=0$. Finalmente, generalizamos este fato para qualquer dobra $f$ associada a uma involução $\varphi$ : temos

$$
f=k \circ f^{0} \circ h^{-1} \text { e } \varphi=h \circ \varphi^{0} \circ h^{-1}
$$

com $k$ e $h$ germes de difeomorfismos. Logo

$$
d f(x)=d k\left(f^{0} \circ h^{-1}(x)\right) \circ d\left(f^{0}\right)\left(h^{-1}(x)\right) \circ(d h)^{-1}(x) \quad \text { e } \quad \mathcal{F}(\varphi)=h\left(\mathcal{F}\left(\varphi^{0}\right)\right) .
$$

Observamos que $x \in \Sigma(f)$ se, é somente se, $h^{-1}(x) \in \Sigma\left(f^{0}\right)=\mathcal{F}\left(\varphi^{0}\right)$. Portanto, $x \in \Sigma(f)$ se, é somente se, $x \in h\left(\mathcal{F}\left(\varphi^{0}\right)\right)=\mathcal{F}(\varphi)$.

Proposição 1.7: Dada uma involução $\varphi$ em $\left(\mathbb{R}^{n}, 0\right) \operatorname{com} \operatorname{codim} \mathcal{F}(\varphi)=1$, existe uma dobra $f:\left(\mathbb{R}^{n}, 0\right) \mapsto\left(\mathbb{R}^{n}, 0\right)$, associada a $\varphi$.

Demonstração: $\quad$ Se $\operatorname{codim} \mathcal{F}(\varphi)=1, \varphi$ é conjugada à involução $\varphi^{0}\left(x_{1}, \ldots, x_{n}\right)=$ $\left(-x_{1}, x_{2}, \ldots, x_{n}\right)$, isto é, existe $h$ germe de difeomorfismo tal que $\varphi=h \circ \varphi^{0} \circ h^{-1}$. Definindo a dobra $f=f^{0} \circ h^{-1}$, temos $f$ associada a $\varphi$.

Observação 1.8: A dobra associada a uma involução $\varphi$ não é unicamente determinada. De fato, se $f$ é uma dobra associada a $\varphi$, então qualquer $g \in \mathcal{L} \cdot f$ é também associada a $\varphi$, onde $\mathcal{L}$ é o grupo de equivalências à esquerda.

Lembremos aqui que no estudo de dobras, as involuções $\varphi$ associadas necessariamente satisfazem $\operatorname{codim} \mathcal{F}(\varphi)=1$. Daqui por diante, assumimos, portanto, tal condição quando 
tratamos de involuções associadas a dobras.

Seja $i$ um inteiro fixo, $1 \leq i \leq n$. Considere a involução $\varphi_{i}^{0}$ dada por $\varphi_{i}^{0}\left(x_{1}, \ldots, x_{n}\right)=$ $\left(x_{1}, \ldots,-x_{i}, \ldots, x_{n}\right)$ e a dobra $f_{i}^{0}=\left(x_{1}, \ldots, x_{i}^{2}, \ldots, x_{n}\right)$, associado a $\varphi_{i}^{0}$. Então:

Lema 1.9: Uma dobra $g:\left(\mathbb{R}^{n}, 0\right) \rightarrow\left(\mathbb{R}^{n}, 0\right)$ é associada a $\varphi_{i}^{0}$ se, e só se, g é $\mathcal{L}$ equivalente $a f_{i}^{0}$.

Demonstração: Suponha que $g$ é associada a $\varphi_{i}^{0}$. Então

$$
\begin{aligned}
g \circ \varphi_{i}^{0}\left(x_{1}, \ldots, x_{n}\right) & =g\left(x_{1}, \ldots, x_{n}\right) \\
g\left(x_{1}, \ldots,-x_{i}, \ldots, x_{n}\right) & =g\left(x_{1}, \ldots, x_{i}, \ldots, x_{n}\right) .
\end{aligned}
$$

Da última igualdade podemos ver que $g$ não depende do sinal de $x_{i}$, assim podemos escrever $g$ da seguinte forma:

$$
\begin{aligned}
g\left(x_{1}, \ldots, x_{i}, \ldots, x_{n}\right) & =\left(k_{1}\left(x_{1}, \ldots, x_{i}^{2}, \ldots, x_{n}\right), \ldots, k_{n}\left(x_{1}, \ldots, x_{i}^{2}, \ldots, x_{n}\right)\right) \\
& =\left(k_{1} \circ f^{0}, \ldots, k_{n} \circ f^{0}\right)=k \circ f^{0}
\end{aligned}
$$

onde $k=\left(k_{1}, \ldots, k_{n}\right)$. Como $g$ é uma dobra, então $k$ é um difeomorfismo. Portanto, $g$ é $\mathcal{L}$-equivalente a $f_{i}^{0}$. Reciprocamente se, $g$ é uma dobra $\mathcal{L}$-equivalente a $f_{i}^{0}$, existe um germe de difeomorfismo $k$ tal que $g=k \circ f_{i}^{0}$. Logo

$$
g \circ \varphi_{i}^{0}=k \circ f_{i}^{0} \circ \varphi_{i}^{0}=k \circ f_{i}^{0}=g .
$$

Assim $g$ é uma dobra associada a $\varphi^{0}$.

A seguinte proposição generaliza o Lema 1.9 acima:

Proposição 1.10: Seja $\varphi$ uma involução em $\left(\mathbb{R}^{n}, 0\right)$ e seja h um germe de difeomorfismo de $\left(\mathbb{R}^{n}, 0\right)$ tal que $\varphi=h \circ \varphi_{i}^{0} \circ h^{-1}$. Considere a dobra $f_{i}^{0} \circ h^{-1}$ associada a $\varphi$. Então, a dobra $g:\left(\mathbb{R}^{n}, 0\right) \rightarrow\left(\mathbb{R}^{n}, 0\right)$ é também associada a $\varphi$ se, e só se, g é $\mathcal{L}$-equivalente a $f_{i}^{0} \circ h^{-1}$.

Demonstração: Suponha $g$ uma dobra associada a $\varphi$. É fácil ver que $g \circ h$ é uma dobra associada a $\varphi_{i}^{0}$. Pelo lema anterior, $g \circ h$ é equivalente a $f_{i}^{0}$, o qual quer dizer que existe um difeomorfismo $k$ tal que

$$
g \circ h=k \circ f_{i}^{0},
$$

$\log \mathrm{o}, g=k \circ f_{i}^{0} \circ h^{-1}$. Assim, $g$ é $\mathcal{L}$-equivalente a $f_{i}^{0} \circ h$. Reciprocamente, suponhamos $g \mathcal{L}$ - equivalente a $f_{i}^{0} \circ h^{-1}$. Então, existe $k$ difeomorfismo tal que $g=k \circ f_{i}^{0} \circ h^{-1} \mathrm{e}$

$$
g \circ \varphi=k \circ f_{i}^{0} \circ h^{-1} \circ h \circ \varphi_{i}^{0} \circ h^{-1}=k \circ f_{i}^{0} \circ h^{-1}=g,
$$


o qual termina com a prova desta proposição.

Mais geralmente ainda, podemos reescrever na proposição anterior substituindo $f_{i}^{0} \circ h^{-1}$ por uma dobra associada a $\varphi$ :

Corolário 1.11: Seja $\varphi$ uma involução em $\left(\mathbb{R}^{n}, 0\right)$ e seja $f:\left(\mathbb{R}^{n}, 0\right) \rightarrow\left(\mathbb{R}^{n}, 0\right)$ uma dobra associada a $\varphi$. Então a dobra $g:\left(\mathbb{R}^{n}, 0\right) \rightarrow\left(\mathbb{R}^{n}, 0\right)$ é tambem associada a $\varphi$ se, $e$ só se, $g$ é $\mathcal{L}$-equivalente a $f$.

Demonstração: Seja $g \mathcal{L}$-equivalente a $f$, ou seja, existe um germe de difeomorfismo $k$ tal que $g=k \circ f$. Disto temos que, $g \circ \varphi=k \circ f \circ \varphi=k \circ f=g$ e, portanto, $g$ é associada a $\varphi$. Suponha agora que $g$ é associada a $\varphi$. Como na proposição anterior, $\varphi=h \circ \varphi_{i}^{0} \circ h^{-1}$ e desde que $f$ é também associada a $\varphi$, temos que $f$ e $g$ são $\mathcal{L}$-equivalentes a $f_{i}^{0} \circ h^{-1}$, isto é, existem germes de difeomorfismos $k_{1}$ e $k_{2}$ tais que $g=k_{1} \circ f_{i}^{0} \circ h^{-1}$ e $f=k_{2} \circ f_{i}^{0} \circ h^{-1}$. Logo,

$$
g=k_{1} \circ k_{2}^{-1} \circ k_{2} \circ f_{i}^{0} \circ h^{-1}=k \circ f .
$$

Onde $k=k_{1} \circ k_{2}^{-1}$ e portanto, $g$ é $\mathcal{L}$-equivalente a $f$.

\subsection{Diagramas divergentes de dobras}

Um diagrama de germes com mesma fonte, ou seja, do tipo

$$
\left(f_{1}, \ldots, f_{s}\right):\left(\mathbb{R}^{n}, 0\right) \rightarrow\left(\mathbb{R}^{n} \times \ldots \times \mathbb{R}^{n}, 0\right)
$$

é chamado diagrama divergente. No espaço destes diagramas, o conceito de equivalência é dado pela seguinte definição:

Definição 1.12: Dois diagramas divergentes

$$
\left(f_{1}, \ldots, f_{s}\right):\left(\mathbb{R}^{n}, 0\right) \rightarrow\left(\mathbb{R}^{n} \times \ldots \times \mathbb{R}^{n}, 0\right) \text { e }\left(g_{1}, \ldots, g_{s}\right):\left(\mathbb{R}^{n}, 0\right) \rightarrow\left(\mathbb{R}^{n} \times \ldots \times \mathbb{R}^{n}, 0\right)
$$

são equivalentes se existem germes de difeomorfismo $h, k_{1}, \ldots, k_{s}$ de $\left(\mathbb{R}^{n}, 0\right)$ tais que $g_{i}=$ $k_{i} \circ f_{i} \circ h^{-1}$, para todo $i=1, \ldots, s$.

Nossa atenção é dirigida ao estudo de diagramas divergentes $\left(f_{1}, \ldots, f_{s}\right)$, quando cada $f_{i}$ é uma dobra, $i=1, \ldots, s$.

Definição 1.13: Sejam $\varphi_{1}, \ldots, \varphi_{s}$ involuções em $\left(\mathbb{R}^{n}, 0\right)$ e $\left(f_{1}, \ldots, f_{s}\right)$ um diagrama divergente de dobras. Dizemos que $\left(f_{1}, \ldots, f_{s}\right)$ é associado à $s$-upla $\left(\varphi_{1}, \ldots, \varphi_{s}\right)$, ou $\left(\varphi_{1}, \ldots, \varphi_{s}\right)$ é associada a $\left(f_{1}, \ldots, f_{s}\right)$ se $f_{i}$ é uma dobra associada a $\varphi_{i}$ para todo $i=$ $1, \ldots, s$. 
Agora apresentamos o fundamental resultado que estabelece que a classificação de diagramas divergentes de dobras pode ser reduzido à classificação de $s$-uplas de involuções às quais estão associados.

Teorema 1.14: Seja $\left(f_{1}, \ldots, f_{s}\right)$ um diagrama divergente de dobras associado a $\left(\varphi_{1}, \ldots, \varphi_{s}\right)$ $e\left(g_{1}, \ldots, g_{s}\right)$ um diagrama divergente de dobras associado a $\left(\psi_{1}, \ldots, \psi_{s}\right)$. Então, $\left(f_{1}, \ldots, f_{s}\right)$ $e\left(g_{1}, \ldots, g_{s}\right)$ são equivalentes se, e só se, $\left(\varphi_{1}, \ldots, \varphi_{s}\right)$ e $\left(\psi_{1}, \ldots, \psi_{s}\right)$ são equivalentes.

Demonstração: Suponha $\left(f_{1}, \ldots, f_{s}\right)$ e $\left(g_{1}, \ldots, g_{s}\right)$ equivalentes. Então, existem germes de difeomorfismos $h, k_{1}, \ldots, k_{s}$ de $\left(\mathbb{R}^{n}, 0\right)$ tais que

$$
g_{i}=k_{i} \circ f_{i} \circ h^{-1} \forall i=1, \ldots, s .
$$

Logo a $s$-upla de involuções $\left(h \circ \varphi_{1} \circ h^{-1}, \ldots, h \circ \varphi_{s} \circ h^{-1}\right)$ é associada a $\left(g_{1} \ldots, g_{s}\right)$. Pela unicidade mencionada na Proposição 1.6 segue que

$$
\psi_{i}=h \circ \varphi_{i} h^{-1} \forall i=1, \ldots, s,
$$

e assim temos que $\left(\varphi_{1} \ldots, \varphi_{s}\right)$ e $\left(\psi_{1}, \ldots, \psi_{s}\right)$ são equivalentes.

Reciprocamente, suponha $h$ germe de difeomorfismo de $\left(\mathbb{R}^{n}, 0\right)$ tal que $\psi_{i}=h \circ \varphi_{i} \circ h^{-1}$ para $i=1 \ldots, s$. Então, o diagrama divergente de dobras $\left(f_{1} \circ h^{-1}, \ldots, f_{s} \circ h^{-1}\right)$ é associado a $\left(\psi_{1} \ldots, \psi_{s}\right)$. Pelo Corolário 1.11,

$$
\left(g_{1} \ldots, g_{s}\right) \in \mathcal{L} \cdot\left(f_{1} \circ h^{-1}, \ldots, f_{s} \circ h^{-1}\right)
$$

Portanto, $\left(f_{1}, \ldots, f_{s}\right)$ e $\left(g_{1}, \ldots, g_{s}\right)$ são equivalentes.

Como conseqüência deste resultado, para o caso de diagramas divergentes de dobras $\left(f_{1} \ldots, f_{s}\right)$ temos que, o traço $\operatorname{tr}\left(d\left(\varphi_{1} \circ \ldots \circ \varphi_{s}\right)(0)\right)$ é um invariante por equivalência, onde $\left(\varphi_{1}, \ldots, \varphi_{s}\right)$ é a $s$-upla associada com $\left(f_{1}, \ldots, f_{s}\right)$.

\subsection{Conjuntos de involuções transversais}

Nesta subseção introduzimos a condição fundamental para a classificação de pares de involuções a tratar. Primeramente fazemos uma pausa nas involuções com codimensão do conjuntos dos pontos fixos para tratar pares de involuções com codimensão do conjuntos dos pontos fixos arbitrária a qual será de muita importância no Capítulo 5. Posteriormente exibimos as formas prenormais para $s$-uplas de involuções $\varphi \operatorname{com} \operatorname{codim} \mathcal{F}(\varphi)=1$. 
Definição 1.15: Um conjunto $G_{s}=\left\{\varphi_{1}, \ldots, \varphi_{s}\right\}$ de involuções em $\left(\mathbb{R}^{n}, 0\right), s \leq n$, é transversal se $\mathcal{F}\left(\varphi_{i}\right)$ é transversal a $\mathcal{F}\left(\varphi_{j}\right)$ em 0 para $i \neq j$ e $\operatorname{codim} \cap_{i=1}^{s} T_{0} \mathcal{F}\left(\varphi_{i}\right)=$ $\sum_{i=1}^{s} \operatorname{codim}\left(\mathcal{F}\left(\varphi_{i}\right)\right)$, onde $T_{0} \mathcal{F}\left(\varphi_{i}\right)$ denota o espaço tangente de $\mathcal{F}\left(\varphi_{i}\right)$ em 0 .

Notemos que para $s=2$, isto é, para duas involuções $\varphi_{1}, \varphi_{2}:\left(\mathbb{R}^{n}, 0\right) \rightarrow\left(\mathbb{R}^{n}, 0\right)$, a transversalidade se reduz a

$$
\mathbb{R}^{n}=T_{0} \mathcal{F}\left(\varphi_{1}\right)+T_{0} \mathcal{F}\left(\varphi_{2}\right)
$$

Notemos que no caso de $s$-uplas de involuções lineares não é necessário o tratamento de germe para nossa classificação.

\subsubsection{Formas normais de pares de involuções transversais com di- mensão do subespaço de pontos fixos arbitrária}

Apresentamos a seguinte proposição para cuja prova denotamos por $V^{0} \subset V^{*}$ o conjunto dos funcionais lineares que se anulam sobre o subespaço vetorial $V$ de $\mathbb{R}^{n}$, isto é,

$$
V^{0}=\{f ; f(p)=0, p \in V\}
$$

Da álgebra linear temos que $\operatorname{dim} V^{0}=\operatorname{codim} V($ Veja [9, p. 101]).

Proposição 1.16: Sejam $\varphi_{1}, \varphi_{2}$ involuções lineares transversais em $\mathbb{R}^{n}$ tais que $\operatorname{dim} \mathcal{F}\left(\varphi_{1}\right)$ $=r e \operatorname{dim} \mathcal{F}\left(\varphi_{2}\right)=s$. Então, $\left(\varphi_{1}, \varphi_{2}\right)$ é linearmente equivalente ao par $\left(\psi_{1}, \psi_{2}\right)$ tal que $\mathcal{F}\left(\psi_{1}\right)$ e $\mathcal{F}\left(\psi_{2}\right)$ são dados por $x_{1}=\ldots x_{n-r}=0$ e $x_{n-r+1}=\ldots x_{2 n-r-s}=0$, respectivamente. Portanto, $\psi_{1}$ e $\psi_{2}$ têm matrizes do tipo

$$
\psi_{1}=\left(\begin{array}{c|c|c}
-\mathrm{I}_{n-r} & 0 & 0 \\
\hline A_{2} & \mathrm{I}_{n-s} & 0 \\
\hline A_{3} & 0 & \mathrm{I}_{r+s-n}
\end{array}\right), \psi_{2}=\left(\begin{array}{c|c|c}
\mathrm{I}_{n-r} & B_{1} & 0 \\
\hline 0 & -\mathrm{I}_{n-s} & 0 \\
\hline 0 & B_{3} & \mathrm{I}_{r+s-n}
\end{array}\right) .
$$

Demonstração: Primeiro provamos que $\left(\varphi_{1}, \varphi_{2}\right)$ é linearmente equivalente ao par $\left(\psi_{1}, \psi_{2}\right)$ tal que $\mathcal{F}\left(\psi_{1}\right)$ e $\mathcal{F}\left(\psi_{2}\right)$ são dados por $x_{1}=\ldots x_{n-r}=0$ e $x_{n-r+1}=\ldots x_{2 n-r-s}=0$ respectivamente. Desde que $\operatorname{dim} \mathcal{F}\left(\varphi_{1}\right)=r$ e $\operatorname{dim} \mathcal{F}\left(\varphi_{2}\right)=s$, por um resultado da álgebra linear ([9, p. 109]), os subespaços anuladores de $\mathcal{F}\left(\varphi_{1}\right)$ e $\mathcal{F}\left(\varphi_{2}\right)$ são tais que

$$
\operatorname{codim} \mathcal{F}\left(\varphi_{1}\right)^{0}=n-r \text { e } \operatorname{codim} \mathcal{F}\left(\varphi_{2}\right)^{0}=n-s
$$

e se pegamos $\left\{f_{1}, \ldots, f_{n-r}\right\}$ base de $\mathcal{F}\left(\varphi_{1}\right)^{0}$ e $\left\{f_{n-r+1}, \ldots, f_{2 n-r-s}\right\}$ base de $\mathcal{F}\left(\varphi_{2}\right)^{0}$ temos 
que

$$
\begin{aligned}
& \mathcal{F}\left(\varphi_{1}\right)=\left\{p \in \mathbb{R}^{n} ; f_{1}(p)=\cdots=f_{n-r}(p)=0\right\}, \\
& \mathcal{F}\left(\varphi_{2}\right)=\left\{p \in \mathbb{R}^{n} ; f_{n-r+1}(p)=\cdots=f_{2 n-r-s}(p)=0\right\} .
\end{aligned}
$$

Desde que $\varphi_{1}$ e $\varphi_{2}$ são tranversais,

$$
\operatorname{codim} \mathcal{F}\left(\varphi_{1}\right) \cap \mathcal{F}\left(\varphi_{2}\right)=\operatorname{codim} \mathcal{F}\left(\varphi_{1}\right)+\operatorname{codim} \mathcal{F}\left(\varphi_{2}\right)=2 n-r-s \leq n
$$

Afirmação: $\left\{f_{1}, \ldots, f_{2 n-r-s}\right\}$ é linearmente independente em $\left(\mathbb{R}^{n}\right)^{*}$. De fato, primeiro notemos que

$$
\mathcal{F}\left(\varphi_{1}\right) \cap \mathcal{F}\left(\varphi_{2}\right)=\left\{p \in \mathbb{R}^{n} ; f_{i}(p)=0,1 \leq i \leq 2 n-r-s\right\}
$$

Além disso, codim $\mathcal{F}\left(\varphi_{1}\right) \cap \mathcal{F}\left(\varphi_{2}\right)=\operatorname{dim}\left(\mathcal{F}\left(\varphi_{1}\right) \cap \mathcal{F}\left(\varphi_{2}\right)\right)^{*}=2 n-r-s$, portanto, $f_{1}, \ldots, f_{2 n-r-s}$ são linearmente independentes. Agora pegamos as funções $f_{2 n-r-s+1}, \ldots, f_{n}$ de modo que $f_{1}, \ldots, f_{n}$ formam uma base de $\left(\mathbb{R}^{n}\right)^{*}$, onde para esta base existe uma base $\left\{v_{1}, \ldots, v_{n}\right\}$ de $\mathbb{R}^{n}$. Considere a base canônica $\left\{\pi_{1}, \ldots, \pi_{n}\right\}$ de $\left(\mathbb{R}^{n}\right)^{*}$, onde $\pi_{i}$ denota a projeção canônica $\pi_{i}\left(x_{1}, \ldots, x_{n}\right)=x_{i}$, para $i=1, \ldots, n$. Agora, seja $h^{-1}$ a matriz de mudança de base de $\left\{e_{1}, \ldots, e_{n}\right\}$ para $\left\{v_{1}, \ldots, v_{n}\right\}$, a qual induz o operador invertível $\left(h^{-1}\right)^{*}$ que leva elementos da base $\left\{f_{1}, \ldots, f_{n}\right\}$ na base $\left\{\pi_{1}, \ldots, \pi_{n}\right\}$ de $\left(\mathbb{R}^{n}\right)^{*}$, que esta definida da seguinte forma:

$$
\left(h^{-1}\right)^{*} f_{i}=f_{i} \circ h^{-1}=\pi_{i}, \quad i=1, \ldots, n .
$$

Tomando $\psi_{i}=h \circ \varphi_{i} \circ h^{-1}$ para $i=1,2,\left(\varphi_{1}, \varphi_{2}\right)$ é equivalente a $\left(\psi_{1}, \psi_{2}\right)$. Agora falta provar que

$$
\begin{gathered}
\mathcal{F}\left(\psi_{1}\right)=\left\{p \in \mathbb{R}^{n} ; x_{1}=\ldots=x_{n-r}=0\right\} \mathrm{e} \\
\mathcal{F}\left(\psi_{2}\right)=\left\{p \in \mathbb{R}^{n} ; x_{n-r+1}=\ldots=x_{2 n-r-s}=0\right\} .
\end{gathered}
$$

Se pegamos $v \in \mathcal{F}\left(\psi_{1}\right)=h\left(\mathcal{F}\left(\varphi_{1}\right)\right)$, temos que $v=h(u)$ com $u \in \mathcal{F}\left(\varphi_{1}\right)$. Seja $f_{i} \in$ $\left(\mathcal{F}\left(\varphi_{1}\right)\right)^{0}$. Vejamos que

$$
\pi_{i}(v)=f_{i} \circ h^{-1}(v)=f_{i}(u)=0,
$$

o qual prova que

$$
\mathcal{F}\left(\psi_{1}\right) \subseteq\left\{p \in \mathbb{R}^{n} ; x_{1}=\ldots=x_{n-r}=0\right\} .
$$

Agora, se $v \in\left\{p \in \mathbb{R}^{n} ; x_{1}=\ldots=x_{n-r}=0\right\}$, então $0=\pi_{i}(v)=f_{i} \circ h^{-1}(v)$ para $1 \leq i \leq$ $n-r$. Assim, $v \in h\left(\mathcal{F}\left(\varphi_{1}\right)\right)=\mathcal{F}\left(\psi_{1}\right)$. De forma similar se prova a segunda igualdade, o qual termina de provar a afirmação. 
Sabemos que $\psi_{1}\left(0, \ldots, 0, x_{n-r+1}, \ldots, x_{n}\right)=\left(0, \ldots, 0, x_{n-r+1}, \ldots, x_{n}\right)$. Se escrevemos $\psi_{1}$ em blocos

$$
\psi_{1}=\left(\begin{array}{c|c|c}
A_{1} & A_{4} & A_{7} \\
\hline A_{2} & A_{5} & A_{8} \\
\hline A_{3} & A_{6} & A_{9}
\end{array}\right)
$$

onde $A_{1} \in M(n-r), A_{5} \in M(n-s)$ e $A_{9} \in M(r+s-n)$, então

$$
\begin{aligned}
\psi_{1}\left(0, \ldots, 0, x_{n-r+1}, \ldots, x_{2 n-r-s}, \ldots, x_{n}\right) & =\left(A_{4}\left(x_{n-r+1}, \ldots, x_{2 n-r-s}\right)+A_{7}\left(x_{2 n-r-s+1}, \ldots, x_{n}\right),\right. \\
& A_{5}\left(x_{n-r+1}, \ldots, x_{2 n-r-s}\right)+A_{8}\left(x_{2 n-r-s+1}, \ldots, x_{n}\right), \\
& \left.A_{6}\left(x_{n-r+1}, \ldots, x_{2 n-r-s}\right)+A_{9}\left(x_{2 n-r-s+1}, \ldots, x_{n}\right)\right) \\
& =\left(0, \ldots, 0, x_{n-r+1}, \ldots, x_{2 n-r-s}, \ldots, x_{n}\right) .
\end{aligned}
$$

Assim,

$$
\begin{aligned}
A_{4}\left(x_{n-r+1}, \ldots, x_{2 n-r-s}\right)+A_{7}\left(x_{2 n-r-s+1}, \ldots, x_{n}\right) & =(0, \ldots, 0), \\
\left(A_{5}-\mathrm{I}_{n-s}\right)\left(x_{n-r+1}, \ldots, x_{2 n-r-s}\right)+A_{8}\left(x_{2 n-r-s+1}, \ldots, x_{n}\right) & =(0, \ldots, 0), \\
A_{6}\left(x_{n-r+1}, \ldots, x_{2 n-r-s}\right)+\left(A_{9}-\mathrm{I}_{r+s-n}\right)\left(x_{2 n-r-s+1}, \ldots, x_{n}\right) & =(0, \ldots, 0) .
\end{aligned}
$$

Desde que $x_{n-r+1}, \ldots, x_{2 n-r-s}, x_{2 n-r-s+1}, \ldots, x_{n}$ são arbitrários, temos que

$$
\begin{array}{r}
A_{4}=0, \quad A_{7}=0 ; \\
A_{5}=\mathrm{I}_{n-s}, \quad A_{8}=0 ; \\
A_{6}=0, A_{9}=\mathrm{I}_{r+s-n} .
\end{array}
$$

Portanto,

$$
\psi_{1}=\left(\begin{array}{c|c|c}
A_{1} & 0 & 0 \\
\hline A_{2} & \mathrm{I}_{n-s} & 0 \\
\hline A_{3} & 0 & \mathrm{I}_{r+s-n}
\end{array}\right) .
$$

Analogamente fazemos as contas para $\psi_{2}$, logo $\psi_{1}$ e $\psi_{2}$ têm matrizes dos tipos

$$
\psi_{1}=\left(\begin{array}{c|c|c}
A_{1} & 0 & 0 \\
\hline A_{2} & \mathrm{I}_{n-s} & 0 \\
\hline A_{3} & 0 & \mathrm{I}_{r+s-n}
\end{array}\right), \psi_{2}=\left(\begin{array}{c|c|c}
\mathrm{I}_{n-r} & B_{1} & 0 \\
\hline 0 & B_{2} & 0 \\
\hline 0 & B_{3} & \mathrm{I}_{r+s-n}
\end{array}\right) .
$$

Sendo $\psi_{1}$ e $\psi_{2}$ involuções, temos que

$$
\begin{array}{ll}
\text { (a) } A_{1}^{2}=\mathrm{I}_{n-r} & (b) A_{2}+A_{2} A_{1}=0, A_{3}+A_{3} A_{1}=0 \\
(c) B_{2}^{2}=\mathrm{I}_{n-s} & (d) B_{1}+B_{1} B_{2}=0, B_{3}+B_{3} B_{2}=0 .
\end{array}
$$


Observando o item $(a)$ vemos que para provar que $A_{1}=-\mathrm{I}_{n-r}$ é suficiente mostrar que $\mathcal{F}\left(A_{1}\right)=\{0\}$. De fato, se $\left(x_{1}, \ldots, x_{n-r}\right) \in \mathcal{F}\left(A_{1}\right)$, temos

$$
\begin{aligned}
\psi_{1}\left(x_{1}, \ldots, x_{n-r}, 0 \ldots, 0\right)= & \left(A_{1}\left(x_{1}, \ldots, x_{n-r}\right), A_{2}\left(x_{1}, \ldots, x_{n-r}\right), A_{3}\left(x_{1}, \ldots, x_{n-r}\right)\right) \\
& =\left(x_{1}, \ldots, x_{n-r}, A_{2}\left(x_{1}, \ldots, x_{n-r}\right), A_{3}\left(x_{1}, \ldots, x_{n-r}\right)\right) .
\end{aligned}
$$

Do item (b) temos que

$$
\begin{aligned}
& \left(A_{2}+A_{2} A_{1}\right)\left(x_{1}, \ldots, x_{n-r}\right)=0 \Rightarrow A_{2}\left(x_{1}, \ldots, x_{n-r}\right)=0, \\
& \left(A_{3}+A_{3} A_{1}\right)\left(x_{1}, \ldots, x_{n-r}\right)=0 \Rightarrow A_{3}\left(x_{1}, \ldots, x_{n-r}\right)=0 .
\end{aligned}
$$

Assim, $\left(x_{1}, \ldots, x_{n-r}, 0, \ldots, 0\right) \in \mathcal{F}\left(\psi_{1}\right)$, o qual implica que $x_{1}=\ldots=x_{n-r}=0$. Portanto, $\mathcal{F}\left(A_{1}\right)=\{0\}$.

Analogamente, usando $(c)$ e $(d)$ obtemos que $B_{2}=-\mathrm{I}_{n-s}$.

A seguir apresentamos a condição necessaria e suficiente para que dois pares de involuções lineares com codimensão do conjuntos dos pontos fixo arbitrária sejam equivalentes:

Proposição 1.17: Sejam $\left(\psi_{1}, \psi_{2}\right)$ e $\left(\psi_{1}^{\prime}, \psi_{2}^{\prime}\right)$ pares de involuções lineares em $\mathbb{R}^{n}$ com matrizes como em (1.2):

$$
\psi_{1}=\left(\begin{array}{c|c|c}
-\mathrm{I}_{n-r} & 0 & 0 \\
\hline A_{2} & \mathrm{I}_{n-s} & 0 \\
\hline A_{3} & 0 & \mathrm{I}_{r+s-n}
\end{array}\right), \psi_{2}=\left(\begin{array}{c|c|c}
\mathrm{I}_{n-r} & B_{1} & 0 \\
\hline 0 & -\mathrm{I}_{n-s} & 0 \\
\hline 0 & B_{3} & \mathrm{I}_{r+s-n}
\end{array}\right)
$$

$e$

$$
\psi_{1}^{\prime}=\left(\begin{array}{c|c|c}
-\mathrm{I}_{n-r} & 0 & 0 \\
\hline A_{2}^{\prime} & \mathrm{I}_{n-s} & 0 \\
\hline A_{3}^{\prime} & 0 & \mathrm{I}_{r+s-n}
\end{array}\right), \psi_{2}^{\prime}=\left(\begin{array}{c|c|c}
\mathrm{I}_{n-r} & B_{1}^{\prime} & 0 \\
\hline 0 & -\mathrm{I}_{n-s} & 0 \\
\hline 0 & B_{3}^{\prime} & \mathrm{I}_{r+s-n}
\end{array}\right) .
$$

Então $\left(\psi_{1}, \psi_{2}\right)$ e $\left(\psi_{1}^{\prime}, \psi_{2}^{\prime}\right)$ são equivalentes se, e só se, existe $H$ matriz invertível

$$
H=\left(\begin{array}{c|c|c}
\left(\alpha_{1}\right)_{n-r} & & 0 \\
\hline 0 & \left(\alpha_{2}\right)_{n-s} & 0 \\
\hline \delta & \gamma & \beta_{r+s-n}
\end{array}\right)
$$

tal que

$$
\begin{array}{r}
A_{2}^{\prime} \alpha_{1}=\alpha_{2} A_{2}, \\
B_{1}^{\prime} \alpha_{2}=\alpha_{1} B_{1}, \\
A_{3}^{\prime} \alpha_{1}=-2 \delta+\gamma A_{2}+\beta A_{3}, \\
B_{3}^{\prime} \alpha_{2}=\delta B_{1}-2 \gamma+\beta B_{3} .
\end{array}
$$


Demonstração: A prova é conseqüência direta da equivalência de pares de involuções e multiplicação de matrizes.

Agora voltamos para as involuções com codimensão do conjunto dos pontos fixos igual a 1 , que são aquelas que estão relacionadas às dobras.

\subsubsection{Involuções $\varphi \operatorname{com} \operatorname{codim} \mathcal{F}(\varphi)=1$}

Proposição 1.18: Seja $G_{s}=\left\{\varphi_{1}, \ldots, \varphi_{s}\right\}$ um conjunto transversal de involuções lineares em $\mathbb{R}^{n}$. Então, $\left(\varphi_{1}, \ldots, \varphi_{s}\right)$ é linearmente equivalente a $\left(\psi_{1}, \ldots, \psi_{s}\right)$ tal que, para cada $\psi_{i}, \mathcal{F}\left(\psi_{i}\right)$ é dado pela equação $x_{i}=0$ e, portanto, $\psi_{i}$ tem a forma

$$
\psi_{i}\left(x_{1}, \ldots, x_{n}\right)=\left(x_{1}+a_{i 1} x_{i}, \ldots,-x_{i}, \ldots, x_{n}+a_{i n} x_{i}\right)
$$

para constantes $a_{i j}, j \neq i, 1 \leq j \leq n$.

Demonstração: A prova é similar á Proposição 1.16 .

Depois de obter as formas prenormais de $s$-uplas de involuções lineares transversais, prosseguimos a apresentar as formas prenormais dos diagramas divergentes de dobras associadas a estas $s$-uplas.

Proposição 1.19: Seja $G_{s}=\left\{\varphi_{1}, \ldots, \varphi_{s}\right\}$ como na Proposição 1.18. Então qualquer diagrama divergente de dobras $\left(f_{1}, \ldots, f_{s}\right)$ associado a $\left(\varphi_{1}, \ldots, \varphi_{s}\right)$ é equivalente ao diagrama divergente de dobras $\left(g_{1}, \ldots, g_{s}\right)$ associado a $\left(\psi_{1}, \ldots, \psi_{s}\right)$, onde $\psi_{i}$ é dado por $(1.3)$ $e$

$$
g_{i}\left(x_{1}, \ldots, x_{n}\right)=\left(x_{1}+\frac{a_{i 1}}{2} x_{i}, \ldots, x_{i}^{2}, \ldots, x_{n}+\frac{a_{i n}}{2} x_{i}\right) .
$$

Demonstração: $\quad$ Para cada $i=1, \ldots, s$, consideremos o isomorfismo linear $h_{i}$ de $\mathbb{R}^{n}$ dado por

$$
h_{i}\left(x_{1}, \ldots, x_{n}\right)=\left(x_{1}-\frac{a_{i 1}}{2} x_{i}, \ldots, x_{i}, \ldots, x_{n}-\frac{a_{i n}}{2} x_{i}\right),
$$

para o qual

$$
h_{i}^{-1}\left(x_{1}, \ldots, x_{n}\right)=\left(x_{1}+\frac{a_{i 1}}{2} x_{i}, \ldots, x_{i}, \ldots, x_{n}+\frac{a_{i n}}{2} x_{i}\right) .
$$

Então, obtemos $\psi_{i}$ como na proposição anterior:

$$
\begin{aligned}
\psi_{i}=h_{i} \circ \varphi_{i}^{0} \circ h^{-1}\left(x_{1}, \ldots, x_{n}\right) & =h_{i} \circ \varphi_{i}^{0}\left(x_{1}+\frac{a_{i 1}}{2} x_{i}, \ldots, x_{i}, \ldots, x_{n}+\frac{a_{i n}}{2} x_{i}\right) \\
& =h_{i}\left(x_{1}+\frac{a_{i 1}}{2} x_{i}, \ldots,-x_{i}, \ldots, x_{n} \frac{a_{i n}}{2} x_{i}\right) \\
& =\left(x_{1}+a_{i 1} x_{i}, \ldots,-x_{i}, \ldots, x_{n}+a_{i n} x_{i}\right) .
\end{aligned}
$$


Logo $\left(\varphi_{1}^{0}, \ldots, \varphi_{s}^{0}\right)$ é equivalente a $\left(\psi_{1}, \ldots, \psi_{s}\right)$ poe $h$. Assim, a dobra $g_{i}$ definida por $g_{i}=f_{i}^{0} \circ h_{i}^{-1}$ é associada a $\psi_{i}$, pois $g_{i} \circ \psi_{i}=f_{i}^{0} \circ h_{i}^{-1} \circ h_{i} \circ \varphi_{i}^{0} \circ h_{i}^{-1}=g_{i}$ e, portanto, o diagrama divergente de dobras $\left(g_{1}, \ldots, g_{s}\right)$ é associado à $s$-upla de involuções $\left(\psi_{1}, \ldots, \psi_{s}\right)$. Logo, pelo Teorema 1.14 os diagramas divergentes de dobras $\left(g_{1}, \ldots, g_{s}\right)$ e $\left(f_{1}, \ldots, f_{s}\right)$ são equivalentes.

Os seguintes dois resultados dão as formas normais de $s$-uplas de involuções e dos correspondentes diagramas divergentes de dobras quando o conjunto de involuções é transversal e gera um grupo Abeliano, as involuções não sendo necessariamente lineares.

Teorema 1.20: Se $G_{s}=\left\{\varphi_{1}, \ldots, \varphi_{s}\right\}$ é transversal $e \Delta_{s}=\left\langle\varphi_{1}, \ldots, \varphi_{s}\right\rangle$ é Abeliano, então $\left(\varphi_{1}, \ldots, \varphi_{s}\right)$ é equivalente a $\left(\varphi_{1}^{0}, \ldots, \varphi_{s}^{0}\right)$, onde

$$
\varphi_{i}^{0}\left(x_{1}, \ldots, x_{n}\right)=\left(x_{1}, \ldots,-x_{i}, \ldots, x_{n}\right), i=1, \ldots, s .
$$

Demonstração: Pelo Teorema 1.3, $G_{s}=\left\{\varphi_{1}, \ldots, \varphi_{s}\right\}$ é equivalente a $\bar{G}_{s}=\left\{\bar{\varphi}_{1}, \ldots, \bar{\varphi}_{s}\right\}$, com $\bar{\varphi}_{i}$ 's sendo involuções lineares. Como $G_{s}$ é transversal, afirmamos que $\bar{G}_{s}$ é trasversal. De fato, seja $h$ o germe de difeomorfismo tal que $\varphi_{i}=h \circ \bar{\varphi}_{i} \circ h^{-1}$. Temos

$$
h: \mathcal{F}\left(\bar{\varphi}_{i}\right) \rightarrow \mathcal{F}\left(\varphi_{i}\right)
$$

e é fácil ver que $d h(0)\left(T_{0} \mathcal{F}\left(\bar{\varphi}_{i}\right)\right)=d h(0)\left(\mathcal{F}\left(\bar{\varphi}_{i}\right)\right)=T_{0} \mathcal{F}\left(\varphi_{i}\right)$, para cada $1 \leq i \leq s$. E também vejamos que pela tranversalidade de $G_{s}$ e pela linearidade de $\bar{\varphi}_{i}$ :

$$
\begin{aligned}
\operatorname{codim} \bigcap_{i=1}^{s} T_{0} \mathcal{F}\left(\bar{\varphi}_{i}\right) & =\operatorname{codim} \bigcap_{i=1}^{s} \mathcal{F}\left(\bar{\varphi}_{i}\right)=\operatorname{codim} d h(0)\left(\bigcap_{i=1}^{s} \mathcal{F}\left(\bar{\varphi}_{i}\right)\right) \\
& =\operatorname{codim} \bigcap_{i=1}^{s} d h(0)\left(\mathcal{F}\left(\bar{\varphi}_{i}\right)\right)=\operatorname{codim} \bigcap_{i=1}^{s} T_{0} \mathcal{F}\left(\varphi_{i}\right) \\
& =\sum_{i=1}^{s} \operatorname{codim} \mathcal{F}\left(\varphi_{i}\right)=\sum_{i=1}^{s} \operatorname{codim} \mathcal{F}\left(\bar{\varphi}_{i}\right) .
\end{aligned}
$$

Além disso, temos que para $i \neq j$

$$
\begin{aligned}
\mathbb{R}^{n} & =T_{0} \mathcal{F}\left(\varphi_{i}\right)+T_{0} \mathcal{F}\left(\varphi_{j}\right)=d h(0)\left(T_{0} \mathcal{F}\left(\bar{\varphi}_{i}\right)\right)+d h(0)\left(T_{0} \mathcal{F}\left(\bar{\varphi}_{j}\right)\right) \\
& =\operatorname{dh}(0)\left(T_{0} \mathcal{F}\left(\bar{\varphi}_{i}\right)+T_{0} \mathcal{F}\left(\bar{\varphi}_{j}\right)\right)=T_{0} \mathcal{F}\left(\bar{\varphi}_{i}\right)+T_{0} \mathcal{F}\left(\bar{\varphi}_{i}\right)
\end{aligned}
$$

portanto, $\bar{G}_{s}$ é transversal. Mais ainda, $\bar{\Delta}_{s}$ é Abeliano, pois

$$
\bar{\varphi}_{i} \circ \bar{\varphi}_{j}=h^{-1} \circ \varphi_{i} \circ h \circ h^{-1} \circ \varphi_{j} \circ h=h^{-1} \circ \varphi_{j} \circ \varphi_{i} \circ h=\bar{\varphi}_{j} \circ \bar{\varphi}_{i} .
$$

Assim, $\bar{G}_{s}$ é tranversal e Abeliano. Agora podemos argumentar de duas formas que $\bar{\Delta}_{s}$ pode ser tomado com elementos da forma (1.5). A primeira forma é considerar cada $\bar{\varphi}_{i}$ 
como en (1.3) e usar o fato de que $\bar{G}_{s}$ é Abeliano para conseguir que $a_{i j}=0$. A outra é observar que todos os $\bar{\varphi}_{i}$ são diagonalizáveis com $n-1$ autovalores 1 e um autovalor -1. Usando diagonalização simultânea de operadores diagonizáveis e uma matriz de permutação se necessário, obtemos que $\left(\bar{\varphi}_{1}, \ldots, \bar{\varphi}_{s}\right)$ é equivalente a $\left(\varphi_{1}^{0}, \ldots, \varphi_{s}^{0}\right)$.

Pelos teoremas 1.14 e 1.20 temos o seguinte teorema:

Teorema 1.21: $S e G_{s}$ é transversal e $\Delta_{s}=\left\langle\varphi_{1}, \ldots, \varphi_{n}\right\rangle$ é Abeliano, então qualquer diagrama divergente de dobras $\left(f_{1}, \ldots, f_{s}\right)$ associado $a\left(\varphi_{1}, \ldots, \varphi_{s}\right)$ é equivalente ao diagrama divergente de dobras $\left(f_{1}^{0}, \ldots, f_{s}^{0}\right)$, onde

$$
f_{i}^{0}\left(x_{1}, \ldots, x_{n}\right)=\left(x_{1}, \ldots, x_{i}^{2}, \ldots, x_{n}\right), i=1, \ldots, s .
$$




\section{Capítulo}

2

\section{Formas normais de pares de involuções e diagramas divergentes de dobras}

No Capítulo 1 foi desenvolvida a relação entre a classificaçao de diagramas divergentes de dobras $\left(f_{1}, \ldots, f_{s}\right)$ e a classificação de $s$-uplas $\left(\varphi_{1}, \ldots, \varphi_{s}\right)$ de involuções associadas a estes diagramas. Além disso, foi apresentada as formas prenormais desta classificação. Neste capítulo apresentamos as formas normais desta classificação para o caso $n=s=2$ (Teoremas 2.3 e 2.4) as quais nos levam a encontrar as formas normais para o caso $s=2$ e $n \geq 3$ (Teoremas 2.9 e 2.10). Mais ainda, nos teoremas de classificação a ser apresentados, descobrimos que a maioria de formas normais é caracterizada pelo traço da composta destes pares de involuções. Nossa referência básica é [10].

\subsection{Caracterização de órbitas}

Nesta seção caracterizamos as órbitas de diagramas divergentes de dobras $\left(f_{1}, \ldots, f_{s}\right)$ : $\left(\mathbb{R}^{n}, 0\right) \rightarrow\left(\mathbb{R}^{n} \times \ldots \times \mathbb{R}^{n}, 0\right)$ associados à $s$-upla $\left(\varphi_{1}, \ldots, \varphi_{s}\right)$ de involuções lineares transversais em $\left(\mathbb{R}^{n}, 0\right)$ quando o conjunto $\left\{\varphi_{1}, \ldots, \varphi_{s}\right\}$ é transversal.

Na proposição a seguir, assumimos que as $s$-uplas de involuções estão na pré-forma normal (1.3) do capítulo anterior.

Proposição 2.1: Considere as s-uplas de involuções lineares transversais $\left(\psi_{1_{a}}, \ldots, \psi_{s_{a}}\right)$ $e\left(\psi_{1_{b}}, \ldots, \psi_{s_{b}}\right)$, onde

$$
\begin{aligned}
& \psi_{i_{a}}\left(x_{1}, \ldots, x_{n}\right)=\left(x_{1}+a_{i 1} x_{i}, \ldots,-x_{i}, \ldots, x_{n}+a_{i n} x_{i}\right) \\
& \psi_{i_{b}}\left(x_{1}, \ldots, x_{n}\right)=\left(x_{1}+b_{i 1} x_{i}, \ldots,-x_{i}, \ldots, x_{n}+b_{i n} x_{i}\right)
\end{aligned}
$$


para todo $i=1, \ldots, s$. Então, $\left(\psi_{1_{a}}, \ldots, \psi_{s_{a}}\right)$ e $\left(\psi_{1_{b}}, \ldots, \psi_{s_{b}}\right)$ são equivalentes se, e só se, existe uma matriz invertível

$$
H=\left(\begin{array}{ccccccc}
\alpha_{1} & 0 & & & \ldots & & 0 \\
& \alpha_{2} & & & & & \\
0 & & \ddots & \ddots & & & \\
& & & \alpha_{s} & 0 & \ldots & 0 \\
\delta_{s+1} & \gamma_{s+1,2} & \ldots & \gamma_{s+1, s} & \beta_{s+1, s+1} & \ldots & \beta_{s+1, n} \\
\vdots & & \ddots & & & \ddots & \\
\delta_{n} & \gamma_{n 2} & & \gamma_{n s} & \beta_{n, s+1} & & \beta_{n, n}
\end{array}\right)
$$

tal que $\alpha_{1}=1$ e se $1 \leq i, j \leq s, i \neq j$, então

$$
b_{i j}=\frac{\alpha_{j}}{\alpha_{i}} a_{i j}
$$

Se $s+1 \leq j \leq n$, então

$$
b_{1 j}=-2 \delta_{j}+\sum_{k=2}^{s} \gamma_{j k} a_{1 k}+\sum_{k=s+1}^{n} \beta_{j k} a_{1 k}
$$

e para $s+1 \leq j \leq n$ e $2 \leq i \leq s$,

$$
b_{i j}=\frac{1}{\alpha_{i}}\left(\delta_{j} a_{i 1}-2 \gamma_{j i}+\sum_{k=2, k \neq i}^{s} \gamma_{j k} a_{i k}+\sum_{k=s+1}^{n} \beta_{j k} a_{i k}\right) .
$$

Demonstração: As s-uplas de involuções $\left(\psi_{1_{a}}, \ldots, \psi_{s_{a}}\right)$ e $\left(\psi_{1_{b}}, \ldots, \psi_{s_{b}}\right)$ são equivalentes se, e só se, existe $h$ germe de difemorfismo em $\left(\mathbb{R}^{n}, 0\right)$ tal que

$$
\psi_{i_{b}}=h \circ \psi_{i_{a}} \circ h^{-1}
$$

Podemos assumir que $h$ é linear, para isto basta derivar no zero e notar que $d h(0)$ é o difeomorfismo linear tal que $\psi_{i_{b}}=d h(0) \circ \psi_{i_{a}} \circ d h(0)^{-1}$. Logo, para obter a forma $(2.1)$ basta usar multiplicação de matrizes para cada $i=1, \ldots, s$. Finalmente, podemos tomar $\alpha_{1}=1$ desde que se $h$ satisfaz (2.5) para qualquer $\alpha$ não nulo, $\alpha h$ também satisfaz (2.5).

Nas seguintes duas seções usamos a Proposição 2.1 para obter formas normais de pares de involuções lineares transversais em $\mathbb{R}^{n}$. Portanto $s=2$ e $n \geq 2$. Observemos que quando $n>s$, as relações (2.2) até (2.4) mostram que a partição do espaço de parâmetros $\mathbb{R}^{s(n-1)}$ que caracteriza as órbitas das $s$-uplas $\left(\psi_{1}, \ldots, \psi_{s}\right)$ projeta sobre a partição do espaço de parametros $\mathbb{R}^{s(s-1)}$ determinado pelas órbitas de $s$-uplas $\phi_{1}, \ldots, \phi_{s}$ de involuções em $\left(\mathbb{R}^{s}, 0\right)$, com

$$
\phi_{i}=\pi_{s} \circ \psi_{i} \circ l_{s}
$$


onde $l_{s}\left(x_{1}, \ldots, x_{n}\right)=\left(x_{1}, \ldots, x_{s}, 0, \ldots, 0\right)$ e $\pi_{s}\left(y_{1}, \ldots, y_{n}\right)=\left(y_{1}, \ldots, y_{s}\right)$. Por esta razão, primeiro estudamos o caso $s=n=2$ (Seção 2.2), a qual é fundamental para o análise dos casos $s=2$ e $n \geq 3$ (Seção 2.3).

Já obtivemos formas normais para o caso Abeliano no contexto não linear (Teorema 1.20). Porém, nas seguintes duas seções argumentamos resultados incluindo pares de involuções lineares transversais gerando um grupo Abeliano para ser completo o estudo. De fato, o caso Abeliano aparece naturalmente no nosso processo de derivar as formas normais.

\subsection{O caso $s=n=2$}

Nesta seção aplicamos os resultados da Seção 2.1 e a Subseção 1.3 .2 para os pares $\left(\varphi_{1}, \varphi_{2}\right)$ de involuções lineares transversais em $\mathbb{R}^{2}$. Na Subseção 2.2.1 apresentamos as formas normais para estes pares de involuções e na Subseção 2.2.2 as formas normais de diagramas divergentes de dobras associadas a estes pares de involuções. Como podemos ver, a maioria de formas normais dependem de um parâmetro, o traço da composta $\varphi_{1} \circ \varphi_{2}$. Esse fato é aplicado na Seção 3.2 na discussão de difeomorfismos reversíveis.

\subsubsection{Formas normais de pares de involuções}

Começamos considerando só pares de involuções $\left(\psi_{1}, \psi_{2}\right)$ como em (1.3) e descrevemos as órbitas no plano $\left(a_{12}, a_{21}\right)$ de parâmetros que aparecem nestes pares. Para este caso particular $s=n=2$ podemos descrever a partição deste plano determinado por órbitas. Para isto, consideramos os pares $\left(\psi_{1_{a}}, \psi_{2_{a}}\right)$ e $\left(\psi_{1_{b}}, \psi_{2_{b}}\right)$, onde

$$
\begin{aligned}
& \psi_{1_{a}}(x, y)=\left(-x, y+a_{12} x\right), \\
& \psi_{2_{a}}(x, y)=\left(x+a_{21} y,-y\right)
\end{aligned}
$$

e

$$
\begin{aligned}
& \psi_{1_{b}}(x, y)=\left(-x, y+b_{12} x\right) \\
& \psi_{2_{b}}(x, y)=\left(x+b_{21} y,-y\right) .
\end{aligned}
$$

Pela Proposição 2.1 temos que $\left(\psi_{1_{a}}, \psi_{2_{a}}\right)$ e $\left(\psi_{1_{b}}, \psi_{2_{b}}\right)$ são equivalentes se, e só se, existe $\alpha$ não nulo tal que

$$
\begin{aligned}
b_{12} & =\alpha a_{12} \\
b_{21} & =\frac{1}{\alpha} a_{21} .
\end{aligned}
$$




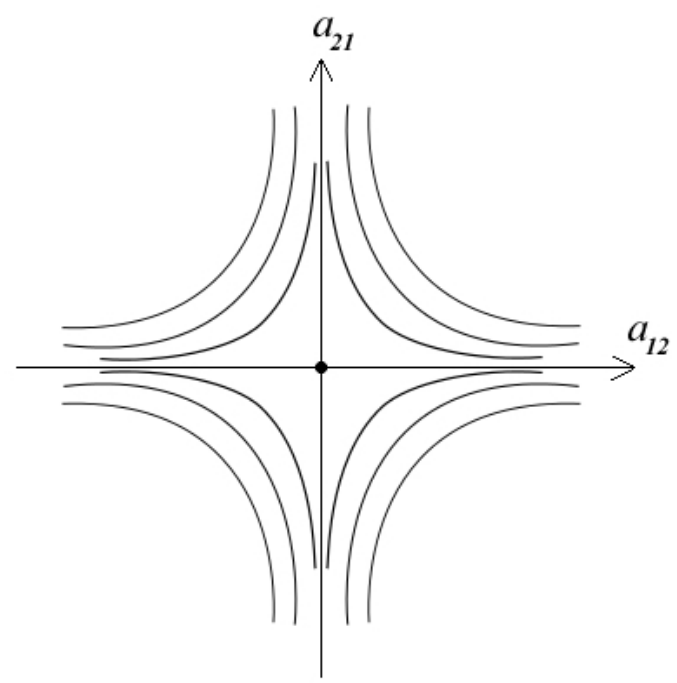

Figura 2.1: Partição do plano $\left(a_{12}, a_{21}\right)$ determinado pelas órbitas de pares de involuções $\left(\psi_{1}, \psi_{2}\right)$.

Portanto, cada órbita determina ou uma hipérbole, ou um eixo menos a origem ou a origem. A origem corresponde aos grupos $\Delta_{2}=\left\langle\psi_{1}, \psi_{2}\right\rangle$ que são Abelianos.

Observemos que cada par $\left(\psi_{1}, \psi_{2}\right)$, com $\Delta_{2}$ não-Abeliano que é equivalente a $\left(\bar{\psi}_{1}, \bar{\psi}_{2}\right)$, onde $\bar{\psi}_{1}(x, y)=\left(-x, y+\bar{a}_{12} x\right)$ e $\bar{\psi}_{2}(x, y)=\left(x+\bar{a}_{21} y,-y\right)$, tem um único ponto representativo $\left(\bar{a}_{12}, \bar{a}_{21}\right)$ que é um ponto fixo arbitrário do eixo- $a_{12}$ (diferente da origem) ou de uma linha paralela a este no plano $\left(a_{12}, a_{21}\right)$. E desde que $\psi_{1} \circ \psi_{2}(x, y)=\left(-x-a_{21} y, a_{12} x+\right.$ $\left.\left(a_{12} a_{21}-1\right) y\right)$, escolhemos

$$
\left(\bar{a}_{12}, \bar{a}_{21}\right)=\left\{\begin{array}{lr}
(1,0) & \text { se } a_{21}=0 \\
\left(2+\operatorname{tr}\left(\psi_{1} \circ \psi_{2}\right), 1\right) & \text { se } a_{21} \neq 0 .
\end{array}\right.
$$

Para a segunda opção, usamos a igualdade

$$
a_{12} a_{21}=2+\operatorname{tr}\left(\psi_{1} \circ \psi_{2}\right) .
$$

Essas ideias são representadas esquematicamente na Figura 2.2:

Observação 2.2: $\quad$ Dois pares $\left(\psi_{1}, \psi_{2}\right)$ e $\left(\bar{\psi}_{1}, \bar{\psi}_{2}\right)$ com órbitas representadas fora do eixo$a_{12}$ na Figura 2.2 são equivalentes se,e só se, as composições $\psi_{1} \circ \psi_{2}$ e $\bar{\psi}_{1} \circ \bar{\psi}_{2}$ são conjugadas. De fato, a condição necessária segue da hipótese de equivalência de pares de involuções, a suficiência se prova observando que

$$
\left(\bar{a}_{12}, \bar{a}_{21}\right)=\left(2+\operatorname{tr}\left(\psi_{1} \circ \psi_{2}\right), 1\right)=\left(a_{12} a_{21}, 1\right)
$$

e pegando $\alpha=a_{21}$, temos que $\bar{a}_{12}=\alpha a_{12}$ e $\bar{a}_{21}=\alpha^{-1} a_{21}$. 


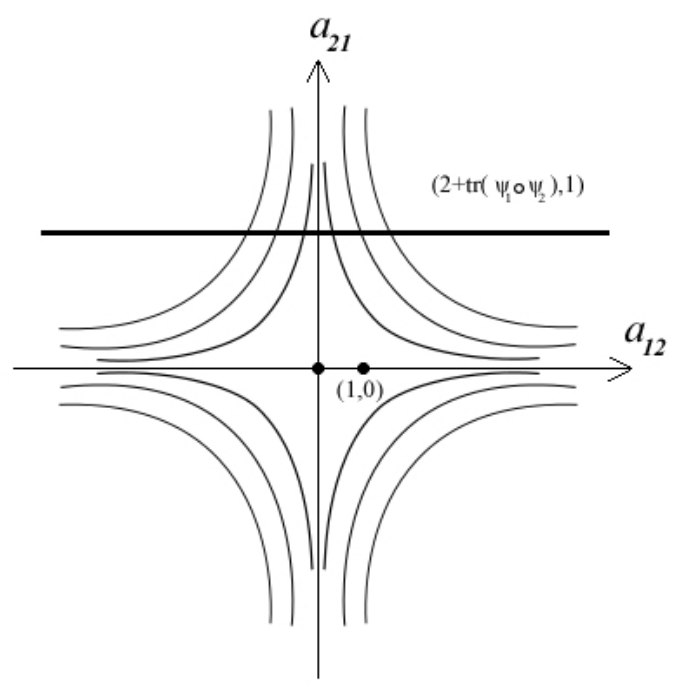

Figura 2.2:

Esta caracterização de órbitas tem aplicações importantes na teoria de sistemas reversíveis que será discutida na Seção 3.2 .

Dada uma involução $\varphi$ linear em $\mathbb{R}^{n}$, consideremos agora o subespaço:

$$
\mathcal{A}(\varphi)=\left\{x \in \mathbb{R}^{n} ; \varphi(x)=-x\right\}
$$

Chamado subespaço antipodal de $\varphi$. Note que $\mathcal{A}(\varphi)=\operatorname{Im}(\varphi-\mathrm{I})$.

Agora observemos que se $a_{21}=0, \psi_{1}(x, y)=\left(-x, a_{12} x+y\right)$ e $\psi_{2}(x, y)=(x,-y)$. Então, $\mathcal{A}\left(\psi_{2}\right)=\{(0, y)\}$ e $\mathcal{F}\left(\psi_{1}\right)=\{(0, y)\}$, e portanto $\mathcal{F}\left(\psi_{1}\right)=\mathcal{A}\left(\psi_{2}\right)$. E se assumimos que $\mathcal{F}\left(\psi_{1}\right)=\mathcal{A}\left(\psi_{2}\right)$, como $\mathcal{F}\left(\psi_{1}\right)=\mathbb{R}\{(0,1)\}$ e $\mathcal{A}\left(\psi_{2}\right)=\mathbb{R}\left\{\left(-a_{21} / 2,1\right)\right\}$, segue que $a_{21}=0$. Portanto,

$$
a_{21}=0 \Leftrightarrow \mathcal{A}\left(\psi_{2}\right)=\mathcal{F}\left(\psi_{1}\right) .
$$

Mais ainda, a igualdade $\mathcal{A}\left(\psi_{2}\right)=\mathcal{F}\left(\psi_{1}\right)$ e o número $2+\operatorname{tr}\left(\psi_{1} \circ \psi_{2}\right)$ são invariantes por conjugação linear simultânea. Portanto, a Proposição 1.18 junto com (2.7) dá o seguinte teorema:

Teorema 2.3: Seja $\left(\varphi_{1}, \varphi_{2}\right)$ um par de involuções lineares transversais em $\mathbb{R}^{2}$. Consideremos o grupo $\Delta_{2}=\left\langle\varphi_{1}, \varphi_{2}\right\rangle$.

(a) Se $\Delta_{2}$ é Abeliano, então $\left(\varphi_{1}, \varphi_{2}\right)$ é equivalente ao par canônico $\left(\varphi_{1}^{0}, \varphi_{2}^{0}\right)$, onde

$$
\varphi_{1}^{0}(x, y)=(-x, y), \varphi_{2}^{0}(x, y)=(x,-y)
$$


(b) Suponha agora que $\Delta_{2}$ seja não-Abeliano. $\operatorname{Se} \mathcal{A}\left(\varphi_{2}\right)=\mathcal{F}\left(\varphi_{1}\right)$, então $\left(\varphi_{1}, \varphi_{2}\right)$ é equivalente a $\left(\bar{\psi}_{1}, \bar{\psi}_{2}\right)$, onde

$$
\bar{\psi}_{1}(x, y)=(-x, y+x), \bar{\psi}_{2}(x, y)=(x,-y)
$$

Se $\mathcal{A}\left(\varphi_{2}\right) \neq \mathcal{F}\left(\varphi_{1}\right)$, então $\left(\varphi_{1}, \varphi_{2}\right)$ é equivalente a $\left(\bar{\psi}_{1}, \bar{\psi}_{2}\right)$, onde

$$
\bar{\psi}_{1}(x, y)=\left(-x, y+\left(2+\operatorname{tr}\left(\psi_{1} \circ \psi_{2}\right)\right) x\right), \bar{\psi}_{2}(x, y)=(x+y,-y) .
$$

\section{Demonstração:}

(a) Segue do Teorema 1.20 .

(b) $\quad$ - Se $\mathcal{A}\left(\varphi_{2}\right)=\mathcal{F}\left(\varphi_{1}\right)$ temos que $a_{21}=0$. Então $\varphi_{1}(x, y)=\left(-x, a_{12} x+y\right)$ e $\varphi_{2}(x, y)=(x,-y)$. Consideremos $\bar{\psi}_{1}(x, y)=(-x, x+y)$ e $\bar{\psi}_{2}(x, y)=(x,-y)$ onde $\bar{a}_{12}=1$ e $\bar{a}_{21}=0$, se pegamos $\alpha=a_{12}$, vemos que $a_{12}=\alpha \cdot 1=\alpha \cdot \bar{a}_{12}$ e $0=a_{21}=\alpha^{-1} \bar{a}_{21}$. Logo, pela Proposição $2.1\left(\varphi_{1}, \varphi_{2}\right)$ é equivalente ao par $\left(\bar{\psi}_{1}, \bar{\psi}_{2}\right)$.

- Se $\mathcal{A}\left(\varphi_{2}\right) \neq \mathcal{F}\left(\varphi_{1}\right)$ temos que $a_{21} \neq 0$. Logo, definimos $\left(\bar{\psi}_{1}, \bar{\psi}_{2}\right)$ como em (2.10) e desde que $a_{12} a_{21}=2+\operatorname{tr}\left(\varphi_{1} \circ \varphi_{2}\right)$, pegando $\alpha=a_{21}$ como no caso anterior, vemos que $\bar{a}_{12}=\alpha a_{12}$ e $\bar{a}_{21}=\alpha^{-1} a_{21}$. Portanto, $\left(\varphi_{1}, \varphi_{2}\right)$ e $\left(\bar{\varphi}_{1}, \bar{\varphi}_{2}\right)$ são equivalentes.

O corolário a seguir é o correspondente ao resultado apresentado na Observação 2.2 para pares gerais de involuções lineares transversais.

Corolário 2.4: Dois pares $\left(\varphi_{1}, \varphi_{2}\right)$ e $\left(\bar{\varphi}_{1}, \bar{\varphi}_{2}\right)$ de involuções lineares transversais em $\mathbb{R}^{2}$, com $\mathcal{A}\left(\varphi_{2}\right) \neq \mathcal{F}\left(\varphi_{1}\right)$ e $\mathcal{A}\left(\bar{\varphi}_{2}\right) \neq \mathcal{F}\left(\bar{\varphi}_{1}\right)$, são equivalentes se, e só se, as composições $\varphi_{1} \circ \varphi_{2}$ $e \bar{\varphi}_{1} \circ \bar{\varphi}_{2}$ são conjugadas.

Demonstração: Para aplicar a Observação 2.2 basta ver que $a_{21} \neq 0$ e $\bar{a}_{21} \neq 0$, o qual quer dizer que os pares $\left(\varphi_{1}, \varphi_{2}\right)$ e $\left(\overline{\varphi_{1}}, \overline{\varphi_{2}}\right)$ têm órbitas representadas fora dos eixos $a_{12} \mathrm{e}$ $\bar{a}_{12}$ respectivamente.

\subsubsection{Formas normais de diagramas divergentes de dobras}

Nesta subseção apresentamos a classificação de diagramas divergentes de dobras

$$
\left(f_{1}, f_{2}\right):\left(\mathbb{R}^{2}, 0\right) \rightarrow\left(\mathbb{R}^{2} \times \mathbb{R}^{2}, 0\right)
$$


associados a pares $\left(\varphi_{1}, \varphi_{2}\right)$ de involuções lineares transversais em $\left(\mathbb{R}^{2}, 0\right)$. Esta classificação é obtida através da classificação dos pares de involuções apresentado na Subseção 2.2.1 e é dada pelo seguinte teorema:

Teorema 2.5: $\operatorname{Seja}\left(f_{1}, f_{2}\right):\left(\mathbb{R}^{2}, 0\right) \rightarrow\left(\mathbb{R}^{2} \times \mathbb{R}^{2}, 0\right)$ um diagrama divergente de dobras associado ao par $\left(\varphi_{1}, \varphi_{2}\right)$ de involuções lineares transversais em $\left(\mathbb{R}^{2}, 0\right)$. Consideramos o grupo $\Delta_{2}=\left\langle\varphi_{1}, \varphi_{2}\right\rangle$.

(a) Se $\Delta_{2}$ é Abeliano, então $\left(f_{1}, f_{2}\right)$ é equivalente ao diagrama divergente de dobras canônico $\left(f_{1}^{0}, f_{2}^{0}\right)$, onde

$$
f_{1}^{0}(x, y)=\left(x^{2}, y\right), f_{2}^{0}(x, y)=\left(x, y^{2}\right)
$$

(b) Suponha $\Delta_{2}$ não-Abeliano. Se $\mathcal{A}\left(\varphi_{2}\right)=\mathcal{F}\left(\varphi_{1}\right)$, então $\left(f_{1}, f_{2}\right)$ é equivalente a $\left(g_{1}, g_{2}\right)$, onde

$$
g_{1}(x, y)=\left(x^{2}, y+\frac{1}{2} x\right), g_{2}(x, y)=\left(x, y^{2}\right) .
$$

Se $\mathcal{A}\left(\varphi_{2}\right) \neq \mathcal{F}\left(\varphi_{1}\right)$, então $\left(f_{1}, f_{2}\right)$ é equivalente a $\left(g_{1}, g_{2}\right)$, onde

$$
g_{1}(x, y)=\left(x^{2}, y+\left(1+\frac{1}{2} \operatorname{tr}\left(\varphi_{1} \circ \varphi_{2}\right)\right) x\right), g_{2}(x, y)=\left(x+\frac{1}{2} y, y^{2}\right) .
$$

Demonstração: Segue do Teorema 2,3 e da Proposição 1,19.

Temos observado que o número $\operatorname{tr}\left(\varphi_{1} \circ \varphi_{2}\right)$ é invariante pela classe de equivâlencia de $\left(f_{1}, f_{2}\right)$. O corolário a seguir diz mais para um tipo especial destes pares:

Corolário 2.6: Sejam $\left(f_{1}, f_{2}\right)$ e $\left(\bar{f}_{1}, \bar{f}_{2}\right)$ dois diagramas divergentes de dobras em $\left(\mathbb{R}^{2}, 0\right)$ associados a pares de involuções lineares transversais $\left(\varphi_{1}, \varphi_{2}\right)$ e $\left(\bar{\varphi}_{1}, \bar{\varphi}_{2}\right)$ respectivamente tal que $\mathcal{A}\left(\varphi_{2}\right) \neq \mathcal{F}\left(\varphi_{1}\right)$ e $\mathcal{A}\left(\bar{\varphi}_{2}\right) \neq \mathcal{F}\left(\bar{\varphi}_{1}\right)$. Então, $\left(f_{1}, f_{2}\right)$ e $\left(\bar{f}_{1}, \bar{f}_{2}\right)$ são equivalentes se, e só se, $\operatorname{tr}\left(\varphi_{1} \circ \varphi_{2}\right)=\operatorname{tr}\left(\bar{\varphi}_{1} \circ \bar{\varphi}_{2}\right)$.

Demonstração: Suponha $\left(f_{1}, f_{2}\right)$ equivalentes. Pelo Teorema 1.14, Corolário 2.4 e pela invariância por conjugação temos que, $\operatorname{tr}\left(\varphi_{1} \circ \varphi_{2}\right)=\operatorname{tr}\left(\bar{\varphi}_{1} \circ \bar{\varphi}_{2}\right)$. Reciprocamente se $\operatorname{tr}\left(\varphi_{1} \circ \varphi_{2}\right)=\operatorname{tr}\left(\bar{\varphi}_{1} \circ \bar{\varphi}_{2}\right)$, pelo Teorema $2.5,\left(f_{1}, f_{2}\right)$ e $\left(\bar{f}_{1}, \bar{f}_{2}\right)$ são equivalentes a $(2.13)$ e portanto, são equivalentes.

\subsection{O caso $s=2, n \geq 3$}

Nesta seção obtemos uma generalização dos resultados da Seção 2.2 dando formas normais de involuções lineares transversais em $\mathbb{R}^{n}, n \geq 3$, e formas normais de diagramas 
divergentes de dobras associadas a estes pares. No caso $n=2$ notamos que quase todas as formas normais dependen de um parâmetro chamado traço da composta das duas involuções. Pode-se notar que o mesmo vale para $n \geq 3$. Porém, temos agora um interessante fenômeno da bifurcação com respeito as $n-2$ novas coordenadas.

\subsubsection{Formas normais de pares de involuções}

Como na seção anterior obtemos formas normais para o caso não-Abeliano, onde consideramos involuções lineares do tipo (1.3)

Proposição 2.7: Consideremos o par $\left(\psi_{1}, \psi_{2}\right)$ de involuções lineares transversais em $\mathbb{R}^{n}, n \geq 3$, dadas por

$$
\begin{aligned}
& \psi_{1}\left(x_{1}, \ldots, x_{n}\right)=\left(-x_{1}, x_{2}+a_{12} x_{1}, x_{3}+a_{13} x_{1}, \ldots, x_{n}+a_{1 n} x_{1}\right), \\
& \psi_{2}\left(x_{1}, \ldots, x_{n}\right)=\left(x_{1}+a_{21} x_{2},-x_{2}, x_{3}+a_{23} x_{2}, \ldots, x_{n}+a_{2 n} x_{2}\right),
\end{aligned}
$$

com $\Delta_{2}=\left\langle\psi_{1}, \psi_{2}\right\rangle$ não-Abeliano, ou seja, $a_{12}^{2}+a_{21}^{2} \neq 0$.

(a) Suponha $a_{12} a_{21} \neq 4$. Se $a_{12}=0$, então $\left(\psi_{1}, \psi_{2}\right)$ é equivalente a $\left(\bar{\psi}_{1}, \bar{\psi}_{2}\right)$, onde

$$
\begin{aligned}
& \bar{\psi}_{1}\left(x_{1}, \ldots, x_{n}\right)=\left(-x_{1}, x_{2}+x_{1}, x_{3}, \ldots, x_{n}\right), \\
& \bar{\psi}_{2}\left(x_{1}, \ldots, x_{n}\right)=\left(x_{1},-x_{2}, x_{3}, \ldots, x_{n}\right) .
\end{aligned}
$$

Se $a_{12} \neq 0$, então $\left(\psi_{1}, \psi_{2}\right)$ é equivalente $a\left(\bar{\psi}_{1}, \bar{\psi}_{2}\right)$, onde

$$
\begin{aligned}
& \bar{\psi}_{1}\left(x_{1}, \ldots, x_{n}\right)=\left(-x_{1}, x_{2}+\left(4-n+\operatorname{tr}\left(\psi_{1} \circ \psi_{2}\right)\right) x_{1}, x_{3}, \ldots, x_{n}\right), \\
& \bar{\psi}_{2}\left(x_{1}, \ldots, x_{n}\right)=\left(x_{1}+x_{2},-x_{2}, x_{3}, \ldots, x_{n}\right) .
\end{aligned}
$$

(b) Suponha $a_{12} a_{21}=4$. Se $\left(a_{23}, \ldots, a_{2 n}\right)=\frac{-a_{21}}{2}\left(a_{13}, \ldots, a_{1 n}\right)$, então $\left(\psi_{1}, \psi_{2}\right)$ é equivalente $a\left(\bar{\psi}_{1}, \bar{\psi}_{2}\right)$, onde

$$
\begin{aligned}
& \bar{\psi}_{1}\left(x_{1}, \ldots, x_{n}\right)=\left(-x_{1}, x_{2}+4 x_{1}, x_{3}, \ldots, x_{n}\right), \\
& \bar{\psi}_{2}\left(x_{1}, \ldots, x_{n}\right)=\left(x_{1}+x_{2},-x_{2}, x_{3}, \ldots, x_{n}\right) .
\end{aligned}
$$

Se $\left(a_{23}, \ldots, a_{2 n}\right) \neq \frac{-a_{21}}{2}\left(a_{13}, \ldots, a_{1 n}\right)$, então $\left(\psi_{1}, \psi_{2}\right)$ é equivalente $a\left(\bar{\psi}_{1}, \bar{\psi}_{2}\right)$, onde

$$
\begin{aligned}
& \bar{\psi}_{1}\left(x_{1}, \ldots, x_{n}\right)=\left(-x_{1}, x_{2}+4 x_{1}, x_{3}, \ldots, x_{n}\right), \\
& \bar{\psi}_{2}\left(x_{1}, \ldots, x_{n}\right)=\left(x_{1}+x_{2},-x_{2}, x_{3}+x_{2}, \ldots, x_{n}\right) .
\end{aligned}
$$

Observação 2.8: Observemos que

$\psi_{1} \circ \psi_{2}=\left(-x_{1}-a_{21} x_{2}, a_{12} x_{1}+\left(a_{21} a_{12}-1\right) x_{2}, a_{13} x_{1}+\left(a_{21} a_{13}+a_{23}\right) x_{2}+x_{3}, \ldots, a_{1 n} x_{1}+\left(a_{21} a_{1 n}\right) x_{2}+x_{n}\right)$, 
onde $\operatorname{tr}\left(\psi_{1} \circ \psi_{2}\right)=-1+a_{21} a_{12}-1+\underbrace{1+\ldots+1}_{n-2 \text { vezes }}$. E portanto,

$$
a_{12} a_{21}=4-n+\operatorname{tr}\left(\psi_{1} \circ \psi_{2}\right)
$$

Note também que esta igualdade é usada para apresentar as formas normais (2.15).

Demonstração: Da Proposição 1.18, seja $\left(\psi_{1_{b}}, \psi_{2_{b}}\right)$ um par de involuções lineares transversais, onde

$$
\begin{aligned}
& \psi_{1_{b}}\left(x_{1}, \ldots, x_{n}\right)=\left(-x_{1}, x_{2}+b_{12} x_{1}, \ldots, x_{n}+b_{1 n} x_{1}\right) \\
& \psi_{2_{b}}\left(x_{1}, \ldots, x_{n}\right)=\left(x_{1}+b_{21} x_{2},-x_{2}, \ldots, x_{n}+b_{2 n} x_{2}\right) .
\end{aligned}
$$

Pela Proposição $2.1\left(\psi_{1}, \psi_{2}\right)$ e $\left(\psi_{1_{b}}, \psi_{2_{b}}\right)$ são equivalentes se, e só se, existe uma matriz invertível

$$
H=\left(\begin{array}{ccccl}
1 & 0 & \ldots & \ldots & 0 \\
0 & \alpha & 0 & \ldots & 0 \\
\delta_{3} & \gamma_{3} & \beta_{33} & \ldots & \beta_{3 n} \\
\vdots & \vdots & \vdots & \ddots & \vdots \\
\delta_{n} & \gamma_{n} & \beta_{n 3} & \ldots & \beta_{n n}
\end{array}\right)
$$

tal que $b_{12}=\alpha a_{12}, b_{21}=\alpha^{-1} a_{21}$ e para $3 \leq j \leq n$

$$
\begin{aligned}
& b_{1 j}=-2 \delta_{j}+\gamma_{j} a_{12}+\sum_{k=3}^{n} \beta_{j k} a_{1 k} \\
& b_{2 j}=\frac{1}{\alpha}\left(\delta_{j} a_{21}-2 \gamma_{j}+\sum_{k=3}^{n} \beta_{j k} a_{2 k}\right),
\end{aligned}
$$

para $\alpha \neq 0$ fixo. Seja $L_{\alpha}: \mathbb{R}^{2 n-4} \rightarrow \mathbb{R}^{2 n-4}$ a tranformação linear definida por $L_{\alpha}\left(\delta_{3}, \ldots, \delta_{n}, \gamma_{3}, \ldots, \gamma_{n}\right)=\left(-2 \delta_{3}+\gamma_{3} a_{12}, \ldots,-2 \delta_{n}+\gamma_{n} a_{12}, \frac{1}{\alpha}\left(\delta_{3} a_{21}-2 \gamma_{3}\right), \ldots, \frac{1}{\alpha}\left(\delta_{n} a_{21}-2 \gamma_{n}\right)\right)$

Para cada isomorfismo linear $\beta: \mathbb{R}^{n-2} \rightarrow \mathbb{R}^{n-2}$, consideremos o vetor $v_{\alpha \beta} \in \mathbb{R}^{n-2} \times \mathbb{R}^{n-2} \cong$ $\mathbb{R}^{2 n-4}$ dado por

$$
v_{\alpha \beta}=\left(\beta\left(a_{13}, \ldots, a_{1 n}\right), \frac{1}{\alpha} \beta\left(a_{23}, \ldots, a_{2 n}\right)\right) \in \mathbb{R}^{n-2} \times \mathbb{R}^{n-2} \cong \mathbb{R}^{2 n-4}
$$


Vejamos também que

$$
\begin{aligned}
\left(b_{13}, \ldots, b_{1 n}, b_{23}, \ldots,\right. & \left.b_{2 n}\right)=\left(-2 \delta_{3}+\gamma_{3} a_{12}+\sum_{k=3}^{n} \beta_{3 k} a_{1 k}, \ldots,-2 \delta_{n}+\gamma_{n} a_{12}+\sum_{k=3}^{n} \beta_{n k} a_{1 k}\right. \\
& \left.\frac{1}{\alpha}\left(\delta_{3} a_{21}-2 \gamma_{3}+\sum_{k=3}^{n} \beta_{3 k} a_{2 k}\right), \ldots, \frac{1}{\alpha}\left(\delta_{n} a_{21}-2 \gamma_{n}+\sum_{k=3}^{n} \beta_{n k} a_{2 k}\right)\right) \\
& =\left(-2 \delta_{3}+\gamma_{3} a_{12}, \ldots,-2 \delta_{n}+\gamma_{n} a_{12}, \frac{1}{\alpha}\left(\delta_{3} a_{21}-2 \gamma_{3}\right), \ldots, \frac{1}{\alpha}\left(\delta_{n} a_{21}-2 \gamma_{n}\right)\right. \\
& \left.+\sum_{k=3}^{n} \beta_{3 k} a_{1 k}, \ldots, \sum_{k=3}^{n} \beta_{n k} a_{1 k}, \frac{1}{\alpha} \sum_{k=3}^{n} \beta_{3 k} a_{2 k}, \ldots, \frac{1}{\alpha} \sum_{k=3}^{n} \beta_{n k} a_{2 k}\right) \\
& =L_{\alpha}\left(\delta_{3}, \ldots, \delta_{n}, \gamma_{3}, \ldots, \gamma_{n}\right)+\left(\beta \times \frac{1}{\alpha} \beta\right)\left(a_{13}, \ldots, a_{1 n}, a_{23}, \ldots, a_{2 n}\right) \\
& =L_{\alpha}\left(\delta_{3}, \ldots, \delta_{n}, \gamma_{3}, \ldots, \gamma_{n}\right)+v_{\alpha \beta} .
\end{aligned}
$$

E portanto, com a notação acima, $\left(\psi_{1}, \psi_{2}\right)$ e $\left(\psi_{1_{b}}, \psi_{2_{b}}\right)$ são equivalentes se, e só se, $\left(b_{12}, b_{21}\right)=\left(\alpha a_{12}, \frac{1}{\alpha} a_{21}\right) \mathrm{e}$

$$
\left(b_{13}, \ldots, b_{1 n}, b_{23}, \ldots, b_{2 n}\right) \in \operatorname{Im}\left(T_{v_{\alpha \beta}} \circ L_{\alpha}\right)
$$

para algum $\alpha \neq 0$, algum difeomorfismo $\beta$ e onde $T_{v_{\alpha \beta}}: \mathbb{R}^{2 n-4} \rightarrow \mathbb{R}^{2 n-4}$ é a translação na direção do vetor $v_{\alpha \beta}$.

Agora, se observamos $L_{\alpha}$ matricialmente

$$
L_{\alpha}=\left(\begin{array}{ccc|ccc}
-2 & & 0 & a_{12} & & 0 \\
& \ddots & & & \ddots & \\
0 & & -2 & 0 & & a_{12} \\
\hline \frac{a_{21}}{\alpha} & & 0 & \frac{-2}{\alpha} & & 0 \\
& \ddots & & \vdots & \ddots & \\
0 & & \frac{a_{21}}{\alpha} & 0 & & \frac{-2}{\alpha}
\end{array}\right)
$$

vemos que $L_{\alpha}$ é isomorfismo linear se, e só se, $a_{12} a_{21} \neq 4$.

(a) Suponha $a_{12} a_{21} \neq 4$. Neste caso $\operatorname{Im}\left(T_{v_{\alpha \beta}} \circ L_{\alpha}\right)=\mathbb{R}^{2 n-4}$ para algum $\alpha \neq 0$ e algum isomorfismo $\beta$ desde que $L_{\alpha}$ e $T_{v_{\alpha \beta}}$ são sobrejetoras.

Afirmação: $\left(\psi_{1}, \psi_{2}\right)$ e $\left(\psi_{1_{b}}, \psi_{2_{b}}\right)$ são equivalentes se, e só se, $\left(\phi_{1}, \phi_{2}\right)$ e $\left(\phi_{1_{b}}, \phi_{2_{b}}\right)$ são equivalentes, onde estes dois últimos pares são dados por

$$
\begin{aligned}
& \phi_{1}(x, y)=\left(-x_{1}, x_{2}+a_{12} x_{1}\right) \text { e } \phi_{2}(x, y)=\left(x_{1}+a_{21} x_{2},-x_{2}\right), \\
& \phi_{1_{b}}(x, y)=\left(-x_{1}, x_{2}+{ }_{12} x_{1}\right) \text { e } \phi_{2_{b}}(x, y)=\left(x_{1}+b_{21} x_{2},-x_{2}\right) .
\end{aligned}
$$

De fato, suponha que $\left(\psi_{1}, \psi_{2}\right)$ é equivalente a $\left(\psi_{1_{b}}, \psi_{2_{b}}\right)$. Então, existe $\alpha \neq 0$ tal que $b_{12}=\alpha a_{12}$ e $b_{21}=\alpha^{-1} a_{21}$ e, portanto, $\left(\phi_{1}, \phi_{2}\right)$ e $\left(\phi_{1_{b}}, \phi_{2_{b}}\right)$ são equivalentes. 
Reciprocamente, suponha que $\left(\phi_{1}, \phi_{2}\right)$ e $\left(\phi_{1_{b}}, \phi_{2_{b}}\right)$ são equivalentes. Então, existe $\alpha \neq 0$ tal que $b_{12}=\alpha a_{12}$ e $b_{21}=\alpha^{-1} a_{21}$. Da hipótese de $a_{12} a_{21} \neq 4$ temos que $\operatorname{Im}\left(T_{v_{\alpha \beta}} \circ L_{\alpha}\right)=\mathbb{R}^{2 n-4}$, o que implica

$$
\left(b_{13}, \ldots, b_{1 n}, b_{23}, \ldots, b_{2 n}\right) \in \mathbb{R}^{2 n-4}=\operatorname{Im}\left(T_{v_{\alpha \beta}} \circ L_{\alpha}\right) \text {. }
$$

Portanto, $\left(\psi_{1}, \psi_{2}\right)$ e $\left(\psi_{1_{b}}, \psi_{2_{b}}\right)$ são equivalentes . Assim, podemos apresentar a formas normais de $\left(\psi_{1}, \psi_{2}\right)$, usando a formas normais das suspensões $\left(\phi_{1}, \phi_{2}\right)$, como segue:

- Se $a_{21}=0$ temos que $\mathcal{A}\left(\phi_{2}\right)=\mathcal{F}\left(\phi_{1}\right)$. Pelo Teorema 2.3, $\left(\phi_{1}, \phi_{2}\right)$ é equivalente a $\left(\varphi_{1}, \varphi_{2}\right)$ onde $\varphi_{1}\left(x_{1}, x_{2}\right)=\left(-x_{1}, x_{2}+x_{1}\right)$ e $\varphi_{2}\left(x_{1}, x_{2}\right)=\left(x_{1},-x_{2}\right)$. Portanto, da afirmação acima $\left(\psi_{1}, \psi_{2}\right)$ é equivalente a (2.14).

- Se $a_{21} \neq 0$, temos que $\mathcal{A}\left(\phi_{2}\right) \neq \mathcal{F}\left(\phi_{1}\right)$. Pelo Teorema 2.3, $\left(\phi_{1}, \phi_{2}\right)$ é equivalente a $\left(\varphi_{1}, \varphi_{2}\right)$, onde $\varphi_{1}\left(x_{1}, x_{2}\right)=\left(-x_{1}, x_{2}+\left(2+\operatorname{tr}\left(\phi_{1} \circ \phi_{2}\right)\right) x_{1}\right)$ e $\varphi_{2}\left(x_{1}, x_{2}\right)=$ $\left(x_{1},-x_{2}\right)$. Finalmente, desde que $2+\operatorname{tr}\left(\phi_{1} \circ \phi_{2}\right)=4-n+\operatorname{tr}\left(\psi_{1} \circ \psi_{2}\right)$, temos que $\left(\psi_{1}, \psi_{2}\right)$ é equivalente a $(2.15)$.

(b) Suponha $a_{12} a_{21}=4$. Se observamos (2.18), então para cada $\alpha \neq 0$, temos que $\operatorname{dim} \operatorname{Im}\left(L_{\alpha}\right)=n-2$ e afirmamos que $\left(u_{1}, \ldots, u_{n-2}, u_{n-1}, \ldots, u_{2 n-4}\right) \in \operatorname{Im}\left(L_{\alpha}\right)$ se, e só se, $\left(u_{n-1}, \ldots, u_{2 n-4}\right)=-\frac{a_{21}}{2 \alpha}\left(u_{1}, \ldots, u_{n-2}\right)$. Para verificar isto, observemos que $\operatorname{Im}\left(L_{\alpha}\right)$ é gerado pelos primeiros $n-2$ vetores coluna de (2.18). Logo,

$$
\operatorname{Im}\left(L_{\alpha}\right)=\mathbb{R}\left\{\left(1,0, \ldots, 0,-\frac{a_{21}}{2 \alpha}, 0, \ldots, 0\right), \ldots,\left(0, \ldots, 0,1,0, \ldots, 0,-\frac{a_{21}}{2 \alpha}\right)\right\}
$$

Assim, dado um isomorfismo $\beta$, temos que

$$
\begin{aligned}
v_{\alpha \beta}=\left(\beta\left(a_{13}, \ldots, a_{1 n}\right), \frac{1}{\alpha} \beta\left(a_{23}, \ldots, a_{2 n}\right)\right) & \in \operatorname{Im}\left(L_{\alpha}\right) \\
\Leftrightarrow & \frac{1}{\alpha} \beta\left(a_{23}, \ldots, a_{2 n}\right)=-\frac{a_{21}}{2 \alpha} \beta\left(a_{13}, \ldots, a_{1 n}\right) \\
\Leftrightarrow & \left(a_{23}, \ldots, a_{2 n}\right)=-\frac{a_{21}}{2}\left(a_{13}, \ldots, a_{1 n}\right) .
\end{aligned}
$$

Então, temos os seguintes casos:

- Se $\left(a_{23}, \ldots, a_{2 n}\right)=-\frac{a_{21}}{2}\left(a_{13}, \ldots, a_{1 n}\right)$, então $v_{\alpha \beta} \in \operatorname{Im}\left(L_{\alpha}\right)$ e portanto

$$
\operatorname{Im}\left(T_{v_{\alpha \beta}} \circ L_{\alpha}\right)=\operatorname{Im}\left(L_{\alpha}\right) ; \text { para algum isomorfismo } \beta
$$

De fato, se pegamos $x \in \operatorname{Im}\left(L_{\alpha}\right), x=L_{\alpha}(a)$, para algum $a \in \mathbb{R}^{2 n-4}$. Desde que $v_{\alpha \beta} \in \operatorname{Im}\left(L_{\alpha}\right), x=L_{\alpha}(a)-v_{\alpha \beta}+v_{\alpha \beta}=L_{\alpha}(a)-L_{\alpha}(b)+v_{\alpha \beta}=L_{\alpha}(a-$ $b)+v_{\alpha \beta}=T_{v_{\alpha \beta}} \circ L_{\alpha}(a-b)$. Portanto, $x \in \operatorname{Im}\left(T_{v_{\alpha \beta}} \circ L_{\alpha}\right)$. Reciprocamente, se 
$x \in \operatorname{Im}\left(T_{v_{\alpha \beta}} \circ L_{\alpha}\right)$, temos que $x=v_{\alpha \beta}+L_{\alpha}(a)=L_{\alpha}(b+a) \in \operatorname{Im}\left(L_{\alpha}\right), \operatorname{com}$ $a, b \in \mathbb{R}^{2 n-4}$. Agora, pegamos $\left(b_{12}, b_{21}\right)=(4,1)$ e $\left(b_{13}, \ldots, b_{1 n}, b_{23}, \ldots, b_{2 n}\right)=$ $(0, \ldots, 0)=-v_{\alpha \beta}+v_{\alpha \beta}=L_{\alpha}(-b)+v_{\alpha \beta} \in \operatorname{Im}\left(T_{v_{\alpha \beta}} \circ L_{\alpha}\right)$. Assim, $\left(\psi_{1}, \psi_{2}\right)$ é equivalente a (2.16).

- Suponhamos $\left(a_{23}, \ldots, a_{2 n}\right) \neq-\frac{a_{21}}{2}\left(a_{13}, \ldots, a_{1 n}\right)$. Então, $v_{\alpha \beta} \notin \operatorname{Im}\left(L_{\alpha}\right)$ para qualquer $\beta$ isomorfismo, e também temos

$$
\cup_{\beta} \operatorname{Im}\left(T_{\alpha \beta} \circ L_{\alpha}\right)=\mathbb{R}^{2 n-4}-\operatorname{Im}\left(L_{\alpha}\right)
$$

Pois se pegamos $u=\left(u_{1}, \ldots, u_{2 n-4}\right) \notin \operatorname{Im}\left(L_{\alpha}\right)$,

$$
\left(u_{n-1}, \ldots, u_{2 n-4}\right)+\frac{a_{21}}{2 \alpha}\left(u_{1}, \ldots, u_{n-2}\right) \neq 0,
$$

e desde que $\left(a_{23}, \ldots, a_{2 n}\right)+\frac{a_{21}}{2}\left(a_{13}, \ldots, a_{1 n}\right) \neq 0$, podemos definir um isomorfismo $\beta$ em $\mathbb{R}^{n-2}$ tal que $\frac{1}{\alpha} \beta\left(\left(a_{23}, \ldots, a_{2 n}\right)+\frac{a_{21}}{2}\left(a_{13}, \ldots, a_{1 n}\right)\right)=\left(u_{n-1}, \ldots, u_{2 n-4}\right)+\frac{a_{21}}{2 \alpha}\left(u_{1}, \ldots, u_{n-2}\right)$.

Disto temos que $\left(u_{n-1}, \ldots, u_{2 n-4}\right)-\frac{1}{\alpha} \beta\left(a_{23}, \ldots, a_{2 n}\right)=-\frac{a_{21}}{2 \alpha}\left(\left(u_{1}, \ldots, u_{n-2}\right)-\beta\left(a_{13}, \ldots, a_{1 n}\right)\right)$, o qual significa que $u-v_{\alpha \beta}=\left(u_{1}, \ldots, u_{2 n-4}\right)-v_{\alpha \beta} \in \operatorname{Im}\left(L_{\alpha}\right)$. Portanto, $u \in \operatorname{Im}\left(T_{v_{\alpha \beta}} \circ L_{\alpha}\right)$. Se pegamos $u \in \cup_{\beta} \operatorname{Im}\left(T_{v_{\alpha \beta}} \circ L_{\alpha}\right)$, temos que $u=L_{\alpha}(v)+v_{\alpha \beta}$ para algum $\beta$ isomorfismo em $\mathbb{R}^{n-2}$. Como $v_{\alpha \beta} \notin \operatorname{Im}\left(L_{\alpha}\right)$, temos $u \notin \operatorname{Im}\left(L_{\alpha}\right)$, o qual termina de verificar (2.19).

Agora, para concluir que (2.17) é a forma normal é suficiente pegar $\left(b_{12}, b_{21}\right)=(4,1)$ e $\left(b_{13}, \ldots, b_{1 n}, b_{23}, \ldots, b_{2 n}\right)=(0, \ldots, 0,1,0, \ldots, 0)$, onde $(0, \ldots, 0,1,0, \ldots, 0) \notin \operatorname{Im}\left(L_{\alpha}\right)$. De fato, suponhamos que $(0, \ldots, 0,1,0, \ldots, 0) \in$ $\operatorname{Im}\left(L_{\alpha}\right)$. Então, existe $c=\left(c_{1}, \ldots, c_{2 n-4}\right) \in \mathbb{R}^{2 n-4}$ tal que $L_{\alpha}\left(c_{1}, \ldots, c_{2 n-4}\right)=$ $(0, \ldots, 0,1,0, \ldots, 0)$ com representação matricial:

$$
L_{\alpha}(c)=\left(\begin{array}{ccc|ccc}
-2 & & 0 & a_{12} & & 0 \\
& \ddots & & & \ddots & \\
0 & & -2 & 0 & & a_{12} \\
\hline \alpha^{-1} a_{21} & & 0 & -2 \alpha^{-1} & & 0 \\
& \ddots & & \vdots & \ddots & \\
0 & & \alpha^{-1} a_{21} & 0 & & -2 \alpha^{-1}
\end{array}\right)\left(\begin{array}{c}
c_{1} \\
\vdots \\
c_{n-2} \\
c_{n-1} \\
\vdots \\
c_{2 n-4}
\end{array}\right)=\left(\begin{array}{c}
0 \\
\vdots \\
0 \\
1 \\
\vdots \\
0
\end{array}\right)
$$

a partir da qual obtemos o sistema de equações 


$$
\left\{\begin{array}{l}
-c_{1}+a_{12} c_{n-1}=0 \\
\frac{a_{21}}{\alpha} c_{1}-\frac{2}{\alpha} c_{n-1}=1 .
\end{array}\right.
$$

Observemos que o cojunto solução é vazio desde que $a_{12} a_{21}=4$. Assim, não existe $c$ tal que $L_{\alpha}(c)=(0, \ldots, 0,1, \ldots, 0)$. E portanto, $(0, \ldots, 0,1, \ldots, 0) \in$ $\operatorname{Im}\left(T_{v_{\alpha \beta}} \circ L_{\alpha}\right)$ para algum $\beta$ isomorfismo de $\mathbb{R}^{n-2}$.

A Proposição 2.16 nos dá formas normais de involuções lineares transversais em $\mathbb{R}^{n}$, $n \geq 3$, basada nas condições de pares da forma (1.3). Podemos agora explicitar o fenômeno de bifurcação com respeito as $n-2$ novas coordenadas. Isto acontece quando $a_{12} a_{21}=4$ e $\left(a_{23}, \ldots, a_{2 n}\right)=\frac{-a_{21}}{2}\left(a_{13}, \ldots, a_{1 n}\right)$, correspondente à forma normal (2.4).

Da Observação 2.8, temos que

$$
a_{12} a_{21}=4-n+\operatorname{tr}\left(\psi_{1} \circ \psi_{2}\right)
$$

Agora observemos que

$$
a_{21}=0 \Leftrightarrow \mathcal{A}\left(\psi_{2}\right) \subset \mathcal{F}\left(\psi_{1}\right)
$$

$\mathrm{e}$, se $a_{12} a_{21}=4$,

$$
\left(a_{23}, \ldots, a_{2 n}\right) \neq \frac{-a_{21}}{2}\left(a_{13}, \ldots, a_{1 n}\right) \Leftrightarrow \mathcal{A}\left(\psi_{2}\right)=\mathcal{A}\left(\psi_{1}\right)
$$

De fato, suponhamos $a_{21}=0$. Então $\psi_{2}\left(x_{1}, \ldots, x_{n}\right)=\left(-x_{1},-x_{2}, x_{3}+a_{23} x_{2}, \ldots, x_{n}+\right.$ $\left.a_{2 n} x_{2}\right)$ e, se pegamos $u=\left(u_{1}, \ldots, u_{n}\right) \in \mathcal{A}\left(\psi_{2}\right)$, temos

$$
\psi_{2}\left(u_{1}, \ldots, u_{n}\right)=\left(u_{1},-u_{2}, u_{3}+a_{23} u_{2}, \ldots, u_{n}+a_{2 n} u_{2}\right)=\left(-u_{1}, \ldots,-u_{n}\right),
$$

o qual implica que a primeira coordenada de $u$ é zero. Logo,

$$
\psi_{1}\left(0, u_{2}, \ldots, u_{n}\right)=\left(0, u_{2}, \ldots, u_{n}\right)
$$

Portanto, $u \in \mathcal{F}\left(\psi_{1}\right)$. Reciprocamente, se pegamos $u \in \mathcal{A}\left(\psi_{2}\right) \subset \mathcal{F}\left(\psi_{1}\right)$, temos que $\psi_{1} \circ \psi_{2}(u)=-u$ e igualando a primeira coordenada, concluimos que $a_{21}=0$. E (2.22) resulta de um cálculo direto ao fazer as contas em (1.3).

O lado direito das três afirmações acima são invariantes sob conjugação simultânea linear. Com este fato, podemos reescrever a Proposição 2.7 em termos de condições sobre pares gerais de involuções lineares transversais. Então temos:

Teorema 2.9: Seja $\left(\varphi_{1}, \varphi_{2}\right)$ um par de involuções lineares transversais em $\mathbb{R}^{n}, n \geq 3$. Consideramos o grupo $\Delta_{2}=\left\langle\varphi_{1}, \varphi_{2}\right\rangle$. 
(a) Se $\Delta_{2}$ é Abeliano, então $\left(\varphi_{1}, \varphi_{2}\right)$ é equivalente ao par canônico $\left(\varphi_{1}^{0}, \varphi_{2}^{0}\right)$, onde

$$
\begin{aligned}
& \varphi_{1}^{0}\left(x_{1}, \ldots, x_{n}\right)=\left(-x_{1}, x_{2}, x_{3}, \ldots, x_{n}\right) \\
& \varphi_{2}^{0}\left(x_{1}, \ldots, x_{n}\right)=\left(x_{1},-x_{2}, x_{3}, \ldots, x_{n}\right) .
\end{aligned}
$$

(b) Suponha $\Delta_{2}$ não-Abeliano. Consideramos dois casos:

(b1) Suponha $\operatorname{tr}\left(\varphi_{1} \circ \varphi_{2}\right) \neq n$. Se $\mathcal{A}\left(\varphi_{2}\right) \subset \mathcal{F}\left(\varphi_{1}\right)$, então $\left(\varphi_{1}, \varphi_{2}\right)$ é equivalente a $\left(\bar{\psi}_{1}, \bar{\psi}_{2}\right)$ dado por (2.14). Se $\mathcal{A}\left(\varphi_{2}\right) \not \subset \mathcal{F}\left(\varphi_{1}\right)$, então $\left(\varphi_{1}, \varphi_{2}\right)$ é equivalente a $\left(\bar{\psi}_{1}, \bar{\psi}_{2}\right)$ dado por

$$
\begin{aligned}
& \bar{\psi}_{1}\left(x_{1}, \ldots, x_{n}\right)=\left(-x_{1}, x_{2}+\left(4-n+\operatorname{tr}\left(\varphi_{1} \circ \varphi_{2}\right)\right) x_{1}, x_{3}, \ldots, x_{n}\right), \\
& \bar{\psi}_{2}\left(x_{1}, \ldots, x_{n}\right)=\left(x_{1}+x_{2},-x_{2}, x_{3}, \ldots, x_{n}\right) .
\end{aligned}
$$

(b2) Suponha $\operatorname{tr}\left(\varphi_{1} \circ \varphi_{2}\right)=n$. Se $\mathcal{A}\left(\varphi_{2}\right)=\mathcal{A}\left(\varphi_{1}\right)$, então $\left(\varphi_{1}, \varphi_{2}\right)$ é equivalente a $\left(\bar{\psi}_{1}, \bar{\psi}_{2}\right)$ dado por (2.16). Se $\mathcal{A}\left(\varphi_{2}\right) \neq \mathcal{A}\left(\varphi_{1}\right)$, então $\left(\varphi_{1}, \varphi_{2}\right)$ é equivalente a $\left(\bar{\psi}_{1}, \bar{\psi}_{2}\right)$ dado por $(2.17)$

\subsubsection{Formas normais de diagramas divergentes de dobras}

Vamos agora passar para diagramas divergentes de dobras. O teorema de classificação é dado abaixo e segue diretamente dos Teoremas 1.14 e 2.9 e da Proposição 1.19:

Teorema 2.10: Seja $\left(f_{1}, f_{2}\right):\left(\mathbb{R}^{n}, 0\right) \rightarrow\left(\mathbb{R}^{n} \times \mathbb{R}^{n}, 0\right)$ um diagrama divergente de dobras associado a um par $\left(\varphi_{1}, \varphi_{2}\right)$ de involuções lineares transversais em $\left(\mathbb{R}^{n}, 0\right)$. Consideremos o grupo $\Delta_{2}=\left\langle\varphi_{1}, \varphi_{2}\right\rangle$.

(a) Se $\Delta_{2}$ é Abeliano, então $\left(f_{1}, f_{2}\right)$ é equivalente ao diagrama canônico de dobras $\left(f_{1}^{0}, f_{2}^{0}\right)$, onde

$$
f_{1}^{0}\left(x_{1}, \ldots, x_{n}\right)=\left(x_{1}^{2}, x_{2}, x_{3}, \ldots, x_{n}\right), f_{2}^{0}\left(x_{1}, \ldots, x_{n}\right)=\left(x_{1}, x_{2}^{2}, x_{3}, \ldots, x_{n}\right)
$$

(b) Suponha agora que $\Delta_{2}$ é não-Abeliano. Consideramos dois casos:

(b1) Suponha $\operatorname{tr}\left(\varphi_{1} \circ \varphi_{2}\right) \neq n$. Se $\mathcal{A}\left(\varphi_{2}\right) \subset \mathcal{F}\left(\varphi_{1}\right)$, então $\left(f_{1}, f_{2}\right)$ é equivalente a $\left(g_{1}, g_{2}\right)$ dado por

$$
\begin{aligned}
& g_{1}\left(x_{1}, \ldots, x_{n}\right)=\left(x_{1}^{2}, x_{2}+\frac{1}{2} x_{1}, x_{3}, \ldots, x_{n}\right), \\
& g_{2}\left(x_{1}, \ldots, x_{n}\right)=\left(x_{1}, x_{2}^{2}, x_{3}, \ldots, x_{n}\right) .
\end{aligned}
$$


Se $A\left(\varphi_{2}\right) \not \subset \mathcal{F}\left(\varphi_{1}\right)$, então $\left(f_{1}, f_{2}\right)$ é equivalente a $\left(g_{1}, g_{2}\right)$ dado por:

$$
\begin{aligned}
& g_{1}\left(x_{1}, \ldots, x_{n}\right)=\left(x_{1}^{2}, x_{2}+\frac{1}{2}\left(4-n+\operatorname{tr}\left(\varphi_{1} \circ \varphi_{2}\right)\right) x_{1}, x_{3}, \ldots, x_{n}\right), \\
& g_{2}\left(x_{1}, \ldots, x_{n}\right)=\left(x_{1}+\frac{1}{2} x_{2}, x_{2}^{2}, x_{3}, \ldots, x_{n}\right) .
\end{aligned}
$$

(b2) Suponha $\operatorname{tr}\left(\varphi_{1} \circ \varphi_{2}\right)=n$. Se $\mathcal{A}\left(\varphi_{2}\right)=\mathcal{A}\left(\varphi_{1}\right)$, então $\left(f_{1}, f_{2}\right)$ é equivalente a $\left(g_{1}, g_{2}\right)$ dado por

$$
\begin{aligned}
& g_{1}\left(x_{1}, \ldots, x_{n}\right)=\left(x_{1}^{2}, x_{2}+2 x_{1}, x_{3}, \ldots, x_{n}\right), \\
& g_{2}\left(x_{1}, \ldots, x_{n}\right)=\left(x_{1}+\frac{1}{2} x_{2}, x_{2}^{2}, x_{3}, \ldots, x_{n}\right) .
\end{aligned}
$$

$\operatorname{Se} \mathcal{A}\left(\varphi_{2}\right) \neq \mathcal{A}\left(\varphi_{1}\right)$, então $\left(f_{1}, f_{2}\right)$ é equivalente a $\left(g_{1}, g_{2}\right)$ dado por

$$
\begin{aligned}
& g_{1}\left(x_{1}, \ldots, x_{n}\right)=\left(x_{1}^{2}, x_{2}+2 x_{1}, x_{3}, \ldots, x_{n}\right), \\
& g_{2}\left(x_{1}, \ldots, x_{n}\right)=\left(x_{1}+\frac{1}{2} x_{2}, x_{2}^{2}, x_{3}, \ldots, x_{n}\right) .
\end{aligned}
$$


30 Capítulo 2. Formas normais de pares de involuções e diagramas divergentes de dobras 


\section{Capítulo 3 \\ Diagramas divergentes de dobras, pares de involuções e sistemas dinâmicos}

Existe uma forte relação entre campos vetoriais descontinuos e mapeamentos de diagramas divergentes. Por exemplo, em [19] é discutida a classificação de singularidades de campos vetoriais descontinuos através da teoria de singularidades e essa relação.

Neste capítulo apresentamos aplicações dos resultados vistos no Capítulo 2 e temos como referência [10]. Mais especificamente, apresentamos uma relação explícita entre campos vetorias descontinuos e diagramas divergentes de dobras associados a um par de involuções transversais. E também aplicamos os resultados de classificação aos sistemas dinâmicos discretos reversíveis. Neste último, descrevemos os difeomorfismos lineares reversiveis dados pela composta de duas involuções no plano. E finalmente damos a descrição de difeomorfismos dados pela composta de uma classe de involuções em $\left(\mathbb{R}^{n}, 0\right)$, $n \geq 3$.

\subsection{Campos vetoriais descontínuos}

Nesta seção ilustramos o aparecimento de involuções e dobras no estudo sistemas descontínuos. Seja $Z$ campo de vetores suave em $\left(\mathbb{R}^{n}, 0\right)$ dado por

$$
Z\left(x_{1}, \ldots, x_{n+1}\right)= \begin{cases}X\left(x_{1}, \ldots, x_{n+1}\right) & \text { se } x_{n+1}>0 \\ Y\left(x_{1}, \ldots, x_{n+1}\right) & \text { se } x_{n+1}<0\end{cases}
$$

onde $X$ e $Y$ são germes de campos vetoriais suaves em $\left(\mathbb{R}^{n+1}, 0\right) \operatorname{com} X(0) \neq 0$ e $Y(0) \neq 0$. Isto significa que $Z$ pode ter descontinuidade no hiperplano $H=\left\{x \in \mathbb{R}^{n+1} ; x_{n+1}=0\right\}$. Consideremos a seguinte situação: Sejam $\gamma_{X}(0, t)$ e $\gamma_{Y}(0, t)$ as órbitas de $X$ e $Y$ passando 
por 0. Suponha que

$$
\begin{gathered}
\gamma_{X}(0,0)=\gamma_{Y}(0,0)=0, \\
\pi_{n+1}\left(\frac{d}{d t} \gamma_{X}(0,0)\right)=\pi_{n+1}\left(\frac{d}{d t} \gamma_{Y}(0,0)\right)=0, \\
\pi_{n+1}\left(\frac{d^{2}}{d t^{2}} \gamma_{X}(0,0)\right)<0 \text { e } \pi_{n+1}\left(\frac{d^{2}}{d t^{2}} \gamma_{Y}(0,0)\right)>0,
\end{gathered}
$$

onde $\pi_{n+1}$ é a projeção de $\mathbb{R}^{n+1}$ no eixo- $x_{n+1}$. Sejam $H_{X}^{\perp}$ e $H_{Y}^{\perp}$ seções transversais às órbitas de $X$ e $Y$ respectivamente. Para cada $p \in(H, 0)$, existe um único $t=t(p)$ em $(\mathbb{R}, 0)$ tal que a órbita $\gamma_{X}(p, t)$ de $X$ que passa por $p$ e encontra $H_{X}^{\perp}$ no ponto $q=\gamma_{X}(p, t(p))$. Analogamente, existe um único $\widetilde{t}=\widetilde{t}(p)$ em $(\mathbb{R}, 0)$ tal que a órbita $\gamma_{Y}(p, t)$ de $Y$ que passa por $H_{Y}^{\perp}$ no ponto $p$ encontra $H_{Y}^{\perp}$ no ponto $\widetilde{q}=\gamma_{Y}(p, \widetilde{t}(p))$. Assim, definimos o diagrama divergente:

$$
\left(f_{X}, f_{Y}\right):(H, 0) \rightarrow\left(H_{X}^{\perp}, 0\right) \times\left(H_{Y}^{\perp}, 0\right),
$$

onde $f_{X}(p)=q$ e $f_{Y}(p)=\widetilde{q}$. Da definição de $f_{X}$ e $f_{Y}$ e de (3.2) até (3.4) podemos concluir que $f_{X}$ e $f_{Y}$ são dobras de $(H, 0)$.

Notemos que os pontos singulares de $f_{X}$ ( respectivamente $f_{Y}$ ) são os pontos de $H$ onde $X$ (respectivamente $Y$ ) é tangente a $H$. Chamemos de $M_{X}$ ( respectivamente $M_{Y}$ ), a esses conjuntos e assumimos que são subvariedade transversais em $(H, 0)$. Definimos agora os germes de difeomorfismos $\varphi_{X}$ e $\varphi_{Y}$ em $(H, 0)$ da seguinte forma:

$$
\varphi_{X}\left(x_{1}, \ldots, x_{n}, 0\right)= \begin{cases}\left(x_{1}, \ldots, x_{n}, 0\right) & \text { se }\left(x_{1}, \ldots, x_{n}, 0\right) \in M_{X} \\ p & \text { se }\left(x_{1}, \ldots, x_{n}, 0\right) \notin M_{X}\end{cases}
$$

onde $p$ é o ponto que está na órbita de $X$ que passa por $\left(x_{1}, \ldots, x_{n}, 0\right)$ e encontra $H$. Analogamente, definimos $\varphi_{Y}$. Temos que $\varphi_{X}$ e $\varphi_{Y}$ são involuções em $(H, 0), \mathcal{F}\left(\varphi_{X}\right)=M_{X}$ e $\mathcal{F}\left(\varphi_{Y}\right)=M_{Y}$. Logo, podemos ver também que $\left(\varphi_{X}, \varphi_{Y}\right)$ está associado ao diagrama divergente de dobras $\left(f_{X}, f_{Y}\right)$ e $\Sigma\left(f_{X}\right)=M_{X}, \Sigma\left(f_{Y}\right)=M_{Y}$. Finalmente, tomando cartas , podemos supor, localmente que $H=H_{X}^{\perp}=H_{Y}^{\perp}=\mathbb{R}^{n}$. Portanto, o diagrama $\left(f_{X}, f_{Y}\right)$ dá origem a um diagrama divergente de dobras associado ao par $\left(\varphi_{X}, \varphi_{Y}\right)$ de involuções transversais. 


\subsection{Difeomorfismos reversíveis}

A referência básica para o estudo descrito nesta seção é [10]. Dada uma involução $\varphi$ em $\left(\mathbb{R}^{n}, 0\right)$, dizemos que um germe de difeomorfismo $F:\left(\mathbb{R}^{n}, 0\right) \rightarrow\left(\mathbb{R}^{n}, 0\right)$ é $\varphi$-reversível se

$$
\varphi \circ F=F^{-1} \circ \varphi
$$

Note que para duas involuções quaisquer $\varphi_{1}$ e $\varphi_{2}$ em $\left(\mathbb{R}^{n}, 0\right)$, a composição $F=\varphi_{1} \circ \varphi_{2}$ é $\varphi_{1}$-reversível. Reciprocamente, um germe de difeomorfismo $F$ com uma simetria reversível involutória $\varphi_{1}$ sempre pode ser escrita como composição de involuções:

$$
F=\varphi_{1} \circ \varphi_{2}
$$

Onde $\varphi_{2}=F^{-1} \circ \varphi_{1}$. Como conseqüência, alguns autores têm endereçado o estudo de difeomorfismos $F, \varphi_{1}$-reversíveis ao estudo do par $\left(\varphi_{1}, \varphi_{2}\right)$.

O ponto que queremos notar é que para quaisquer dois pares de involuções $\left(\varphi_{1}, \varphi_{2}\right)$ $\left(\widetilde{\varphi}_{1}, \widetilde{\varphi}_{2}\right)$ que são equivalentes (Definição 1.2), as composições $\varphi_{1} \circ \varphi_{2}$ e $\widetilde{\varphi}_{1} \circ \widetilde{\varphi}_{2}$ geram sistemas reversíveis conjugados, mas a recíproca não é verdade e isto pode ser notado com o seguinte exemplo

$$
\begin{aligned}
& \varphi_{1}\left(x_{1}, \ldots, x_{n}\right)=\left(-x_{1}, x_{2}, \ldots, x_{n}\right), \\
& \varphi_{2}\left(x_{1}, \ldots, x_{n}\right)=\left(x_{1}+a x_{2},-x_{2}, x_{3}, \ldots, x_{n}\right),
\end{aligned}
$$

$\mathrm{e}$

$$
\begin{aligned}
& \widetilde{\varphi_{1}}\left(x_{1}, \ldots, x_{n}\right)=\left(x_{1}+b x_{2},-x_{2}, x_{3}, \ldots, x_{n}\right), \\
& \widetilde{\varphi_{2}}\left(x_{1}, \ldots, x_{n}\right)=\left(-x_{1}-(a+b) x_{2}, x_{2}, x_{3}, \ldots, x_{n}\right),
\end{aligned}
$$

com $a \neq 0$ e $b$ arbitrário. Então, as composições são iguais mas $\left(\varphi_{1}, \varphi_{2}\right)$ e $\left(\widetilde{\varphi_{1}}, \widetilde{\varphi_{2}}\right)$ não são equivalentes pelo Teorema 2.3 e Teorema 2.9 para $n \geq 3$.

Assim, podemos nos perguntar que tipo de restrições o estudo do par $\left(\varphi_{1}, \varphi_{2}\right)$ pode impor ao estudo da dinâmica associada à composição $\varphi_{1} \circ \varphi_{2}$. Os teoremas de classificação do Capítulo 2 revelam que, a menos de equivalência, não existe restrição para a maioria dos pares $\left(\varphi_{1}, \varphi_{2}\right)$ de involuções lineares transversais. De fato, a maioria de formas normais são caracterizadas por $\operatorname{tr}\left(\varphi_{1} \circ \varphi_{2}\right)$ (Ver Teoremas 2.3 e 2.9).

\subsubsection{Caso planar}

Agora apresentamos a descrição de difeomorfismos lineares reversíveis no plano dado pela composição de involuções como no Corolário 2.4. Primeiro lembremos que uma rotação $R_{\theta}, \theta \in[0,2 \pi)$ tem representação matricial

$$
R_{\theta}=\left(\begin{array}{cc}
\cos \theta & -\sin \theta \\
\sin \theta & \cos \theta
\end{array}\right)
$$


e qualquer reflexão é da forma

$$
\left(\begin{array}{cc}
\cos \alpha & \sin \alpha \\
\sin \alpha & -\cos \alpha
\end{array}\right)
$$

$\operatorname{com} \alpha \in<0,2 \pi]$.

Ainda, qualquer rotação $R_{\theta}$ de ângulo $\theta$ pode ser escrita como composição de duas reflexões, isto é, para $\theta \neq 0, \pi$ temos

$$
\left(\begin{array}{ll}
\cos \theta & -\sin \theta \\
\sin \theta & \cos \theta
\end{array}\right)=\left(\begin{array}{ll}
\cos \alpha & \sin \alpha \\
\sin \alpha & -\cos \alpha
\end{array}\right)\left(\begin{array}{ll}
\cos \beta & \sin \beta \\
\sin \beta & -\cos \beta
\end{array}\right)
$$

onde $\alpha+\beta=\theta$. Se $R_{\theta}$ é a composição de duas involuções lineares, ou seja, $R_{\theta}=\varphi_{1} \circ \varphi_{2}$, então estas são necesariamente reflexões, isto segue de notar que casa involução $\varphi_{i}$ com $i=1,2$, pode ser escrita como segue:

$$
\begin{aligned}
& \varphi_{1}=R_{\theta} \circ \varphi_{2} \quad \mathrm{e} \\
& \varphi_{2}=\varphi_{1} \circ R_{\theta},
\end{aligned}
$$

e de verificar que as entradas de cada $\varphi_{1}$ e $\varphi_{2}$ satisfazem (3.6).

Suponha que $F$ seja um difeomorfismo linear dado pela composição de duas involuções, $F=\varphi_{1} \circ \varphi_{2}, \operatorname{com}\left(\varphi_{1}, \varphi_{2}\right)$ nas condições do Corolario 2.4. Desde que $\operatorname{det}(F)=1$, então

$$
-2<\operatorname{tr}(F)<2 \text { se, e só se, } F \text { é conjugada a uma rotação. }
$$

Para provar isto, primeiro suponha $-2<\operatorname{tr}(F)<2$. O polinômio característico de $F$ é

$$
P_{F}(\lambda)=\lambda^{2}-\operatorname{tr}(F) \lambda+\operatorname{det}(F) .
$$

Disto temos que o discriminante do polinômio é negativo e, portanto, $F$ é conjugado a uma rotação.

Reciprocamente, suponha $F=\varphi_{1} \circ \varphi_{2}$ conjugado a uma rotação de ângulo $0 \leq \theta<2 \pi$ :

$$
R_{\theta}=\left(\begin{array}{ll}
\cos \theta & -\sin \theta \\
\sin \theta & \cos \theta
\end{array}\right)
$$

Desde que o traço é invariante por conjugação, $\operatorname{tr}(F)=\operatorname{tr}\left(R_{\theta}\right)$, logo $-2 \leq \operatorname{tr}(F) \leq 2$. Se $\operatorname{tr}(F)=2=2 \cos \theta$, temos que $\theta=0$ o qual implica $F=\mathrm{I}$, portanto $\varphi_{1}=\varphi_{2}$, que contradiz a hipótese de que o par $\left(\varphi_{1}, \varphi_{2}\right)$ é transversal. $\operatorname{Se} \operatorname{tr}(F)=-2=2 \cos \theta$, então $\theta=\pi$ o qual implica $F=-\mathrm{I}$, portanto $\varphi_{1}=-\varphi_{2}$, que contradiz a hipótese $\mathcal{F}\left(\varphi_{1}\right) \neq A\left(\varphi_{2}\right)$ do Corolario 2.4 .

Agora pelo Teorema 2.3, o par $\left(\varphi_{1}, \varphi_{2}\right)$ é representado pelo ponto $(2+\operatorname{tr}(F), 1)$ na reta horizontal $a_{21}=1$ da Figura 2.2, isto é, podemos assumir que as involuções são dadas por

$$
\varphi_{1}(x, y)=(-x, y+(2+\operatorname{tr}(F)) x) \text { e } \varphi_{2}(x, y)=(x+y,-y)
$$


Então, segue-se que $-2<\operatorname{tr}(F)<2$ nos dá o segmento de reta dos pares de involuções equivalentes a pares de reflexões. Se $\operatorname{tr}(F)>2$ ou $\operatorname{tr}(F)<-2$, então $F$ corresponde a um difeomorfismo hiperbólico linear $\varphi_{1}$-reversível. Isto é, os autovalores de $F$ têm módulo fora de $S^{1}$, o qual se verifica observando o polinômio característico de $F$.

A seguir damos a interpretação geométrica de equivalência entre dois pares de reflexões transversais no plano em relação às retas de pontos fixos das reflexões. Note que se $\varphi_{1} \mathrm{e}$ $\varphi_{2}$ são reflexões, então o ângulo no sentido anti-horário a partir da reta $\mathcal{F}\left(\varphi_{2}\right)$ até a reta $\mathcal{F}\left(\varphi_{1}\right)$ é a metade do ângulo de rotação $\theta, R_{\theta}=\varphi_{1} \circ \varphi_{2}$. Portanto, chegamos à importante conclusão de que dois pares de reflexões transversais planares são equivalentes se, e só se, os ângulos entre as retas de pontos fixos de cada par são iguais.

De fato, se dois pares de reflexões $\left(\varphi_{1}, \varphi_{2}\right)$ e $\left(\widetilde{\varphi_{1}}, \widetilde{\varphi_{2}}\right)$ são equivalentes, as compostas $\varphi_{1} \circ \varphi_{2}$ e $\widetilde{\varphi_{1}} \circ \widetilde{\varphi_{2}}$ são conjugadas. Mais ainda, as compostas destes pares são rotações conjugadas e, portanto, têm igual traço. Logo, têm igual ângulo de rotação e igual ângulo entre as retas dos pontos fixos das reflexões. Se os ângulos entre as retas de pontos fixos são iguais, chamemos este ângulo $\theta$ tal que $0<\theta<\pi$. Os traços destas compostas de reflexões são iguais. Provamos que estes pares de reflexões são equivalente da seguinte forma:

- Se $\theta=\pi / 2$, as retas dos pontos fixos $\mathcal{F}\left(\varphi_{1}\right)$ e $\mathcal{F}\left(\widetilde{\varphi_{1}}\right)$ são ortogonais. Logo a composta destas reflexões é - I. Assim, estes pares de reflexões formam grupos Abelianos. Portanto, cada par é equivalente a (2.8) e finalmente são equivalentes.

- Se $0<\theta<\pi$ e $\theta \neq \pi / 2$. Os grupos gerados pelos pares $\left(\varphi_{1}, \varphi_{2}\right)$ e $\left(\widetilde{\varphi_{1}}, \widetilde{\varphi_{2}}\right)$ são não Abelianos e $\mathcal{F}\left(\varphi_{1}\right) \neq \mathcal{A}\left(\varphi_{2}\right)$ e $\mathcal{F}\left(\widetilde{\varphi_{1}}\right) \neq \mathcal{A}\left(\widetilde{\varphi_{2}}\right)$. Assim, pelo Teorema 2.3 estes pares são equivalentes.

\subsubsection{Caso $n \geq 3$}

A seguir, generalizamos o resultado da Subseção 3.2.1 para pares de reflexões em $\mathbb{R}^{n}$, $n \geq 3$. Começamos com a definição:

Definição 3.1: Uma reflexão $\varphi$ em $\mathbb{R}^{n}$ é uma involução linear com $\operatorname{codim} \mathcal{F}(\varphi)=1$ e $\mathcal{A}(\varphi)$ e $\mathcal{F}(\varphi)$ subespaços ortogonais.

Dadas duas reflexões $\varphi_{1}$ e $\varphi_{2}$ em $\mathbb{R}^{n}$, definimos o ângulo entre $\mathcal{F}\left(\varphi_{1}\right)$ e $\mathcal{F}\left(\varphi_{2}\right)$ como o ângulo entre as retas $\mathcal{A}\left(\varphi_{1}\right)$ e $\mathcal{A}\left(\varphi_{2}\right)$.

Observação 3.2: Se $\left(\varphi_{1}, \varphi_{2}\right)$ é um par de reflexões transversais em $\mathbb{R}^{n}$ e $\eta_{1}, \eta_{2}$ os vetores 
diretores das retas $\mathcal{A}\left(\varphi_{1}\right)$ e $\mathcal{A}\left(\varphi_{2}\right)$ respectivamente, consideramos $\left\{v_{1}, \ldots, v_{n-2}\right\}$ base para $\mathcal{F}\left(\varphi_{1}\right) \cap \mathcal{F}\left(\varphi_{2}\right)$. Então, $B=\left\{\eta_{1}, \eta_{2}, v_{1}, \ldots, v_{n-2}\right\}$ é base de $\mathbb{R}^{n}$ e $\varphi_{1} \circ \varphi_{2}$ nesta base tem matriz:

$$
\left(\begin{array}{rrrrr}
\cos \theta & -\sin \theta & 0 & \ldots & 0 \\
\sin \theta & \cos \theta & & & \\
0 & & 1 & & \vdots \\
\vdots & & & \ddots & 0 \\
0 & & \ldots & 0 & 1
\end{array}\right)
$$

onde $\theta / 2$ é o ângulo entre $\mathcal{F}\left(\varphi_{1}\right)$ e $\mathcal{F}\left(\varphi_{2}\right)$.

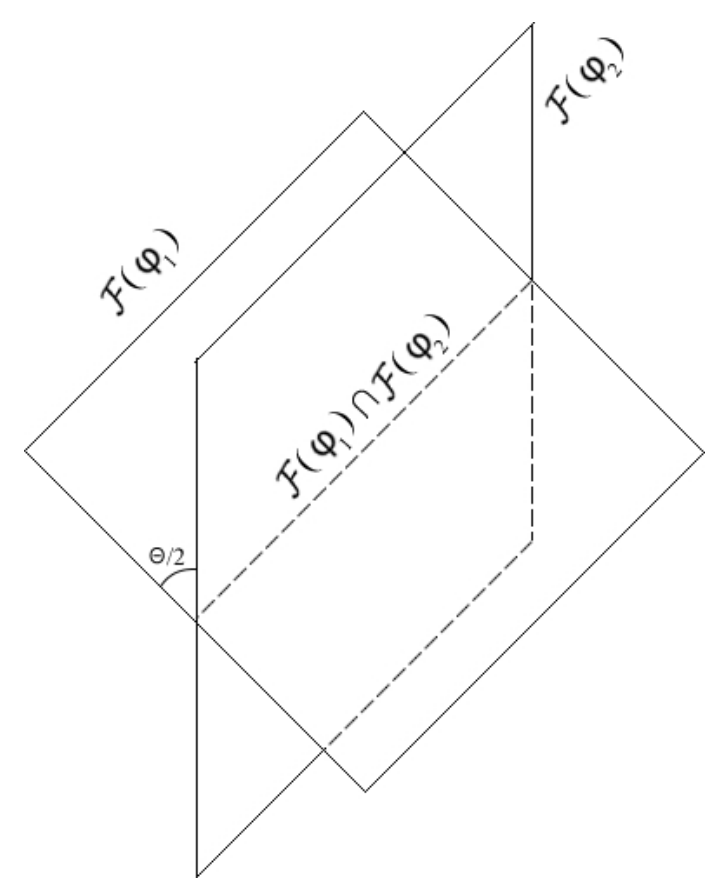

Figura 3.1:

Proposição 3.3: Dois pares de reflexões $\left(\varphi_{1}, \varphi_{2}\right)$ e $\left(\psi_{1}, \psi_{2}\right)$ são equivalentes se, e só se, o ângulo entre $\mathcal{F}\left(\varphi_{1}\right)$ e $\mathcal{F}\left(\varphi_{2}\right)$ e o ângulo entre $\mathcal{F}\left(\psi_{1}\right)$ e $\mathcal{F}\left(\psi_{2}\right)$ são iguais.

Demonstração: $\quad$ Sejam $B=\left\{\eta_{1}, \eta_{2}, v_{1}, \ldots, v_{n-2}\right\}$ e $C=\left\{\nu_{1}, \nu_{2}, u_{1}, \ldots, u_{n-2}\right\}$ bases tomadas como na Observação 3.2 para $\varphi_{1} \circ \varphi_{2}$ e $\psi_{1} \circ \psi_{2}$ respectivamente, com $\alpha$ o ângulo para $\varphi_{1} \circ \varphi_{2}$ e $\beta$ o ângulo para $\psi_{1} \circ \psi_{2}$. Suponhamos que os pares de reflexões $\left(\varphi_{1}, \varphi_{2}\right)$ e $\left(\psi_{1}, \psi_{2}\right)$ são equivalentes. Então, existe $h$ isomorfismo linear tal que $\psi_{1}=h \circ \varphi_{1} \circ h^{-1} \mathrm{e}$ $\psi_{2}=h \circ \varphi_{2} \circ h^{-1}$. Sejam $P$ e $Q$ as matrizes de transição da base canônica para as bases $B$ e $C$ associadas aos pares de reflexões $\left(\varphi_{1}, \varphi_{2}\right)$ e $\left(\psi_{1}, \psi_{2}\right)$ respectivamente. Então,

$$
P \circ \psi_{1} \circ \psi_{2} \circ P^{-1}=P \circ h \circ \varphi_{1} \circ \varphi_{2} \circ h^{-1} \circ P^{-1},
$$


e, portanto,

$$
\left(\psi_{1} \circ \psi_{2}\right)_{C}=P \circ h \circ Q_{-1} \circ\left(\varphi_{1} \circ \varphi_{2}\right)_{B} \circ Q \circ h^{-1} \circ P^{-1}
$$

Assim,

$$
\left(\begin{array}{rcccc}
\cos \alpha & -\sin \alpha & 0 & \ldots & 0 \\
\sin \alpha & \cos \alpha & & & \vdots \\
0 & & 1 & & 0 \\
\vdots & & & \ddots & \\
0 & & \ldots & 0 & 1
\end{array}\right) H=H\left(\begin{array}{rrrrr}
\cos \beta & -\sin \beta & 0 & \ldots & 0 \\
\sin \beta & \cos \beta & & & \vdots \\
0 & & 1 & & \\
& & & \ddots & 0 \\
0 & & \ldots & 0 & 1
\end{array}\right)
$$

onde $H=\left(h_{i j}\right)=P \circ h \circ Q^{-1}$. Pela transversalidade de $\left(\varphi_{1}, \varphi_{2}\right)$ segue que $h_{i j}=0$ para $i=1,2$ e $j=3, \ldots, n$. Pela transversalidade de $\left(\psi_{1}, \psi_{2}\right)$ segue que $h_{i j}=0$ para $i=1 \ldots n$ e $j=1,2$. Então,

$$
\left(\begin{array}{ll}
\cos \alpha & -\sin \alpha \\
\sin \alpha & \cos \alpha
\end{array}\right)\left(\begin{array}{ll}
h_{11} & h_{12} \\
h_{21} & h_{22}
\end{array}\right)=\left(\begin{array}{ll}
h_{11} & h_{12} \\
h_{21} & h_{22}
\end{array}\right)\left(\begin{array}{cc}
\cos \beta & -\sin \beta \\
\sin \beta & \cos \beta
\end{array}\right) .
$$

Disto, temos que $\cos \alpha=\cos \beta$. Então, $\alpha=\beta$ pela transversalidade dos pares $\left(\varphi_{1}, \varphi_{2}\right)$ e $\left(\psi_{1}, \psi_{2}\right)$.

Agora suponhamos que $\alpha=\beta$. Seja $N$ a matriz de transição da base $B$ para a base $C$ tal que $N\left(\eta_{1}\right)=\nu_{1}, N\left(\eta_{2}\right)=\nu_{2}, N\left(v_{1}\right)=u_{1}, \ldots, N\left(v_{n-2}\right)=u_{n-2}$. Pela transversalidade de $\left(\varphi_{1}, \varphi_{2}\right)$ e $\left(\psi_{1}, \psi_{2}\right)$ temos $0<\alpha / 2 \leq \pi / 2$. Se $\alpha / 2=\pi / 2$, então os grupos $\Delta=\left\langle\varphi_{1}, \varphi_{2}\right\rangle$ e $\widetilde{\Delta}=\left\langle\psi_{1}, \psi_{2}\right\rangle$ são Abelianos e portanto os pares $\left(\varphi_{1}, \varphi_{2}\right)$ e $\left(\psi_{1}, \psi_{2}\right)$ são equivalentes. Se $0<\alpha / 2<\pi / 2$, então $n-4<\operatorname{tr}\left(\varphi_{1} \circ \varphi_{2}\right)=\operatorname{tr}\left(\psi_{1} \circ \psi_{2}\right)<n$. Então, pela parte (b) do Teorema $2.9,\left(\varphi_{1}, \varphi_{2}\right)$ e $\left(\psi_{1}, \psi_{2}\right)$ são equivalentes. 
38 Capítulo 3. Diagramas divergentes de dobras, pares de involuções e sistemas dinâmicos 


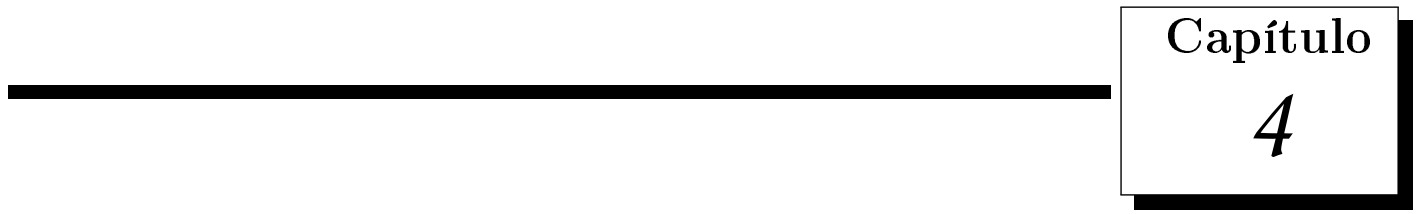 \\ Grupos gerados por um par de involuções e a teoria reversível equivariante}

Inicialmente neste capítulo damos a descrição dos grupos gerados por pares de involuções lineares transversais. O resultado principal é que todo grupo gerado por uma par de involuções é sempre identificado com um grupo diedral finito $D_{m}$ ou infinito $D_{\infty}$. Para o caso planar $(n=2)$ precisamos da representação esquemática apresentada na Figura 2.2 e, do Teorema 2.9 para o caso $n$-dimensional. Os resultados aqui foram obtidos de forma independente. Os cálculos foram feitos de acordo com a forma como se apresenta as involuções no nosso interesse e, apesar de simples, acreditamos que os os resultados não aparecem na literatura. Na segunda parte deste capítulo, apresentamos uma introdução à teoria reversível equivariante a qual é aplicada a grupos específicos gerados por um par de involuções lineares transversais como no Teorema 2.3. Além disso, apresentamos a relação entre os conjuntos apresentados na teoria reversível equivariantes quando os grupos a tratar são conjugados.

Dados o pares de involuções $\left(\varphi_{1}, \varphi_{2}\right)$ e $\left(\tilde{\varphi}_{1}, \tilde{\varphi}_{2}\right)$ equivalentes, sejam $\Delta=\left\langle\varphi_{1}, \varphi_{2}\right\rangle$ e $\tilde{\Delta}=\left\langle\tilde{\varphi}_{1}, \tilde{\varphi}_{2}\right\rangle$ os grupos gerados por estes pares, respectivamente. Seja $h$ o difeomorfismo que conjuga os pares $\left(\varphi_{1}, \varphi_{2}\right)$ e $\left(\tilde{\varphi}_{1}, \tilde{\varphi}_{2}\right)$, isto é, $\tilde{\varphi}_{i}=h \circ \varphi_{i} \circ h^{-1}$, para $i=1,2$. Notemos que cada elemento de $\tilde{\Delta}=\left\langle\tilde{\varphi}_{1}, \tilde{\varphi}_{2}\right\rangle$ é conjugado a um elemento de $\Delta=\left\langle\varphi_{1}, \varphi_{2}\right\rangle$. Mais ainda, estes grupos têm a mesma ordem. Assim, podemos fazer a classificação destes grupos a menos de equivalência. 


\subsection{Grupos gerados por um par de involuções}

Seja $\left(\varphi_{1}, \varphi_{2}\right)$ um par de involuções quaisquer e $\Delta=\left\langle\varphi_{1}, \varphi_{2}\right\rangle$ o grupo gerado pelas involuções $\varphi_{1}$ e $\varphi_{2}$. Lembramos que algebricamente os grupos diedrais são definidos por

$$
D_{m}=\left\langle a, b ; a^{m}=1, b^{2}=1, a b=b a^{-1}\right\rangle, D_{\infty}=\left\langle a, b ; b^{2}=1, a b=b a^{-1}\right\rangle,
$$

onde os elementos $a$ e $b$ são chamados geradores destes grupos. Notemos que o grupo $\Delta=\left\langle\varphi_{1}, \varphi_{2}\right\rangle$ é tambem gerado pelos germes de difeomorfismo $\varphi_{1}$ e $\varphi_{1} \circ \varphi_{2}$, isto é $\Delta=$ $\left\langle\varphi_{1}, \varphi_{1} \circ \varphi_{2}\right\rangle$.

Notemos também que

$$
\left(\varphi_{1} \circ \varphi_{2}\right) \circ \varphi_{1}=\varphi_{1} \circ\left(\varphi_{1} \circ \varphi_{2}\right)^{-1}
$$

Assim, se pegamos $b=\varphi_{1}$ e $a=\varphi_{1} \circ \varphi_{2}$ o grupo $\Delta=\left\langle\varphi_{1}, \varphi_{1} \circ \varphi_{2}\right\rangle$ é ou $\Delta=D_{m}$ ou $\Delta=D_{\infty}$, com $m$ inteiro positivo.

Agora vamos explicitar se $\Delta$ é um $D_{m}$ ou $D_{\infty}$ quando $\left(\varphi_{1}, \varphi_{2}\right)$ é um par de involuções lineares transversais.

\subsubsection{Caso $n=2$}

Começamos por considerar pares $\left(\varphi_{1}, \varphi_{2}\right)$ de involuções lineares transversais no plano. Pelo discutido acima, para a descrição dos grupos $\Delta=\left\langle\varphi_{1}, \varphi_{2}\right\rangle$ só precisamos tomar representantes na Figura 2.2.

1. Se $\left(\varphi_{1}, \varphi_{2}\right)$ está na órbita da origem, então $\Delta$ é Abeliano. Assim, $\Delta \cong D_{2} \cong \mathbb{Z}_{2} \times \mathbb{Z}_{2}$.

2. Se $\left(\varphi_{1}, \varphi_{2}\right)$ está na órbita do ponto $(1,0)$, então, $\left(\varphi_{1}, \varphi_{2}\right)$ é equivalente a $\left(\psi_{1}, \psi_{2}\right)$, onde $\psi_{1}(x, y)=(-x, x+y)$ e $\psi_{2}=(x,-y)$. Então,

$$
\psi_{1} \circ \psi_{2}=\left(\begin{array}{cc}
-1 & 0 \\
1 & -1
\end{array}\right) \text { e }\left(\psi_{1} \circ \psi_{2}\right)^{m}=\left(\begin{array}{cc}
(-1)^{m} & 0 \\
(-1)^{m+1} & (-1)^{m}
\end{array}\right) \text {, }
$$

isto é, $\psi_{1} \circ \psi_{2}$ não tem ordem finita. Logo, $\Delta \cong D_{\infty}$.

3. Suponha $-2<\operatorname{tr}\left(\varphi_{1} \circ \varphi_{2}\right)<2$. Então, $\left(\varphi_{1}, \varphi_{2}\right)$ é equivalente a um par de reflexões $\left(\psi_{1}, \psi_{2}\right)$. Então,

$$
\psi_{1} \circ \psi_{2}=\left(\begin{array}{cc}
\cos \theta & -\sin \theta \\
\sin \theta & \cos \theta
\end{array}\right)
$$

onde $\theta / 2$ é o ângulo entre $\mathcal{F}\left(\psi_{1}\right)$ e $\mathcal{F}\left(\psi_{2}\right)$. Se $\theta$ é múltiplo racional de $\pi$, então existe um menor positivo $m \in \mathbb{Z}$ tal que $\left(\psi_{1} \circ \psi_{2}\right)^{m}=$ I. Neste caso, $\Delta=\left[\varphi_{1}, \varphi_{2}\right] \cong D_{m}$. Caso contrário, $\Delta \cong D_{\infty}$. 
4. Se $\operatorname{tr}\left(\varphi_{1} \circ \varphi_{2}\right)=-2$, então $\left(\varphi_{1}, \varphi_{2}\right)$ é equivalente a $\left(\psi_{1}, \psi_{2}\right)$, onde $\psi_{1}(x, y)=(-x, y)$, $\psi_{2}(x, y)=(x+y,-y) \mathrm{e}$

$$
\psi_{1} \circ \psi_{2}=\left(\begin{array}{rr}
-1 & -1 \\
0 & -1
\end{array}\right)
$$

Desde que a ordem de $\varphi_{1} \circ \varphi_{2}$ é igual à ordem de $\psi_{1} \circ \psi_{2}$, só precisamos saber a ordem de $\psi_{1} \circ \psi_{2}$. Observe que

$$
\left(\psi_{1} \circ \psi_{2}\right)^{m}=\left(\begin{array}{rc}
(-1)^{m} & (-1)^{m} m \\
0 & (-1)^{m}
\end{array}\right) .
$$

Assim, $\psi_{1} \circ \psi_{2}$ não tem ordem finita e $\Delta \cong D_{\infty}$.

5. Se $\operatorname{tr}\left(\varphi_{1} \circ \varphi_{2}\right)=2$, então $\left(\varphi_{1}, \varphi_{2}\right)$ é equivalente a $\left(\psi_{1}, \psi_{2}\right)$, onde $\psi_{1}(x, y)=(-x, y+$ $4 x), \psi_{2}(x, y)=(x+y,-y) \mathrm{e}$

$$
\psi_{1} \circ \psi_{2}=\left(\begin{array}{cc}
-1 & -1 \\
4 & 3
\end{array}\right)
$$

Analogamente, desde que a ordem de $\varphi_{1} \circ \varphi_{2}$ é igual à ordem de $\psi_{1} \circ \psi_{2}$, só precisamos saber o ordem de $\psi_{1} \circ \psi_{2}$. A forma canônica de Jordan de $\psi_{1} \circ \psi_{2}$ é $\left(\begin{array}{ll}1 & 1 \\ 0 & 1\end{array}\right)$ e a forma canônica de Jordan de $\left(\psi_{1} \circ \psi_{2}\right)^{m}$ é $\left(\begin{array}{cc}1 & m \\ 0 & 1\end{array}\right)$. Assim, $\psi_{1} \circ \psi_{2}$ não tem ordem finita e $\Delta \cong D_{\infty}$.

6. Se $\operatorname{tr}\left(\varphi_{1} \circ \varphi_{2}\right)<-2$ ou $\operatorname{tr}\left(\varphi_{1} \circ \varphi_{2}\right)>2$. Então, $\varphi_{1} \circ \varphi_{2}$ é un difeomorfismo linear hiperbólico. A forma canônica de Jordan de $\varphi_{1} \circ \varphi_{2}$ é $\left(\begin{array}{cc}\lambda & 0 \\ 0 & \lambda^{-1}\end{array}\right)$, com $\lambda \in \mathbb{R}$, $|\lambda| \neq 1$, e a forma canônica de Jordan de $\left(\varphi_{1} \circ \varphi_{2}\right)^{m}$ é $\left(\begin{array}{cc}\lambda^{m} & 0 \\ 0 & \lambda^{-m}\end{array}\right)$. Portanto, $\varphi_{1} \circ \varphi_{2}$ não tem ordem finita e $\Delta \cong D_{\infty}$. 


\subsubsection{Caso $n \geq 3$}

Nesta seção descrevemos os grupos $\Delta=\left\langle\varphi_{1}, \varphi_{2}\right\rangle$ para os quais usamos o Teorema 2.9 .

Observação 4.1: Se $\left(\varphi_{1}, \varphi_{2}\right)$ um par de involucões lineares transversais em $\mathbb{R}^{n}$, sabemos que $\operatorname{det}\left(\varphi_{1} \circ \varphi_{2}\right)=1$. Então,

$n-4<\operatorname{tr}\left(\varphi_{1} \circ \varphi_{2}\right)<n$ se, e só se, $\varphi_{1} \circ \varphi_{2}$ é conjugado a $\left(\begin{array}{rc}R_{\theta} & 0 \\ 0 & \mathrm{I}_{n-2}\end{array}\right)$ onde $0<\theta<2 \pi$.

Para a decrição de alguns grupos $\Delta$ usaremos também a Proposição 3.3 :

1. Se $\Delta=\left\langle\varphi_{1}, \varphi_{2}\right\rangle$ é Abeliano então, $\Delta \cong D_{2}=\mathbb{Z}_{2} \times \mathbb{Z}_{2}$.

2. Se $\Delta=\left\langle\varphi_{1}, \varphi_{2}\right\rangle$ é não Abeliano. Como no Teorema 2.8 temos os seguintes casos:

- Suponha $\operatorname{tr}\left(\varphi_{1} \circ \varphi_{2}\right) \neq n$.

(a) Se $\mathcal{A}\left(\varphi_{2}\right) \subset \mathcal{F}\left(\varphi_{2}\right)$. Então, $\left(\varphi_{1}, \varphi_{2}\right)$ é equivalente a $\left(\psi_{1}, \psi_{2}\right)$ dado em $(2.14)$, ou seja,

$$
\begin{aligned}
& \psi_{1}\left(x_{1}, \ldots, x_{n}\right)=\left(-x_{1}, x_{2}+x_{1}, x_{3}, \ldots, x_{n}\right), \\
& \psi_{2}\left(x_{1}, \ldots, x_{n}\right)=\left(x_{1},-x_{2}, x_{3}, \ldots, x_{n}\right) .
\end{aligned}
$$

Logo, temos

$\psi_{1} \circ \psi_{2}=\left(\begin{array}{ccc}-1 & 0 & 0 \\ 1 & -1 & 0 \\ 0 & 0 & \mathrm{I}_{n-2}\end{array}\right)$ e $\left(\psi_{1} \circ \psi_{2}\right)^{m}=\left(\begin{array}{ccc}(-1)^{m} & 0 & 0 \\ (-1)^{m} m & (-1)^{m} & 0 \\ 0 & 0 & \mathrm{I}_{n-2}\end{array}\right)$.

Portanto, $\psi_{1} \circ \psi_{2}$ não tem ordem finita e $\Delta=\left\langle\varphi_{1}, \varphi_{2}\right\rangle \cong D_{\infty}$

(b) Se $\mathcal{A}\left(\varphi_{2}\right) \not \subset \mathcal{F}\left(\varphi_{2}\right)$. Suponha $n-4<\operatorname{tr}\left(\varphi_{1} \circ \varphi_{2}\right)<n$, Então da Observação 4.1 temos que $\varphi_{1} \circ \varphi_{2}$ é conjugado a

$$
\left(\begin{array}{ccc}
\cos \theta & -\sin \theta & 0 \\
\sin \theta & \cos \theta & 0 \\
0 & 0 & \mathrm{I}_{n-2}
\end{array}\right)
$$

Como no caso planar, se $\theta$ é múltiplo racional de $\pi$, então existe um menor positivo $m$ tal que $\left(\psi_{1} \circ \psi_{2}\right)^{m}=\mathrm{I}_{n}$. Portanto, $\Delta=\left\langle\varphi_{1}, \varphi_{2}\right\rangle \cong D_{m}$. Caso contrário, $\Delta \cong D_{\infty}$.

Agora suponha $\operatorname{tr}\left(\varphi_{1} \circ \varphi_{2}\right)=n-4$. Então, $\left(\varphi_{1}, \varphi_{2}\right)$ é equivalente a $\left(\psi_{1}, \psi_{2}\right)$, onde

$$
\begin{aligned}
& \psi_{1}\left(x_{1}, \ldots, x_{n}\right)=\left(-x_{1}, x_{2}, x_{3}, \ldots, x_{n}\right), \\
& \psi_{2}\left(x_{1}, \ldots, x_{n}\right)=\left(x_{1}+x_{2},-x_{2}, x_{3}, \ldots, x_{n}\right) .
\end{aligned}
$$


Logo, temos

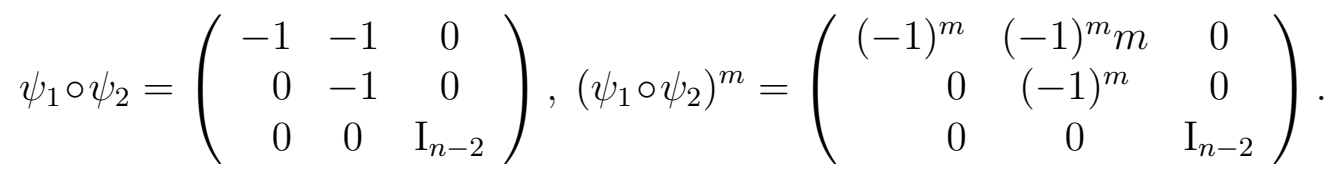

Portanto, $\psi_{1} \circ \psi_{2}$ não tem ordem finita e $\Delta \cong D_{\infty}$.

Se $\operatorname{tr}\left(\varphi_{1} \circ \varphi_{2}\right)<n-4$ ou $\operatorname{tr}\left(\varphi_{1} \circ \varphi_{2}\right)>n$ as formas canônicas de Jordan de $\varphi_{1} \circ \varphi_{2}$ e $\left(\varphi_{1} \circ \varphi_{2}\right)^{m}$ são

$$
\left(\begin{array}{ccc}
\lambda & 0 & 0 \\
0 & \lambda^{-1} & 0 \\
0 & 0 & \mathrm{I}_{n-2}
\end{array}\right),\left(\begin{array}{ccc}
\lambda^{m} & 0 & 0 \\
0 & \lambda^{-m} & 0 \\
0 & 0 & \mathrm{I}_{n-2}
\end{array}\right)
$$

respectivamente, onde $\lambda \in \mathbb{R}$ e $|\lambda| \neq 1$. Portanto, $\varphi_{1} \circ \varphi_{2}$ não tem ordem finita e $\Delta \cong D_{\infty}$.

- Suponha $\operatorname{tr}\left(\varphi_{1} \circ \varphi_{2}\right)=n$.

(a) Se $\mathcal{A}\left(\varphi_{2}\right)=\mathcal{A}\left(\varphi_{2}\right)$ então, $\left(\varphi_{1}, \varphi_{2}\right)$ é equivalente a $\left(\psi_{1}, \psi_{2}\right)$, onde

$$
\begin{aligned}
& \psi_{1}\left(x_{1}, \ldots, x_{n}\right)=\left(-x_{1}, x_{2}+4 x_{1}, x_{3}, \ldots, x_{n}\right), \\
& \psi_{2}\left(x_{1}, \ldots, x_{n}\right)=\left(x_{1}+x_{2},-x_{2}, x_{3}+x_{2}, x_{4}, \ldots, x_{n}\right) .
\end{aligned}
$$

Logo, temos

$$
\psi_{1} \circ \psi_{2}=\left(\begin{array}{cccc}
-1 & -1 & 0 & 0 \\
4 & 3 & 0 & \vdots \\
0 & 1 & 1 & 0 \\
0 & \ldots & 0 & \mathrm{I}_{n-3}
\end{array}\right) .
$$

As formas canônicas de Jordan de $\varphi_{1} \circ \varphi_{2}$ e $\left(\varphi_{1} \circ \varphi_{2}\right)^{m}$ são

$$
\left(\begin{array}{cccc}
-1 & -1 & 0 & 0 \\
0 & 1 & 1 & \vdots \\
\vdots & 0 & 1 & 0 \\
0 & \ldots & 0 & \mathrm{I}_{n-3}
\end{array}\right), \quad\left(\begin{array}{cccc}
1 & m & a & 0 \\
0 & 1 & m & \vdots \\
\vdots & 0 & 1 & 0 \\
0 & \ldots & 0 & \mathrm{I}_{n-3}
\end{array}\right)
$$

Onde $a=\sum_{i=1}^{m-1} i$. Portanto, $\psi_{1} \circ \psi_{2}$ não tem ordem finita e $\Delta \cong D_{\infty}$.

(b) Se $\mathcal{A}\left(\varphi_{2}\right) \neq \mathcal{A}\left(\varphi_{2}\right)$ então, $\left(\varphi_{1}, \varphi_{2}\right)$ é equivalente a $\left(\psi_{1}, \psi_{2}\right)$, onde

$$
\begin{aligned}
& \psi_{1}\left(x_{1}, \ldots, x_{n}\right)=\left(-x_{1}, x_{2}+4 x_{1}, x_{3}, \ldots, x_{n}\right), \\
& \psi_{2}\left(x_{1}, \ldots, x_{n}\right)=\left(x_{1}+x_{2},-x_{2}, x_{3}+x_{2}, x_{4}, \ldots, x_{n}\right) .
\end{aligned}
$$


Logo, temos

$$
\psi_{1} \circ \psi_{2}=\left(\begin{array}{ccc}
-1 & -1 & 0 \\
4 & 3 & 0 \\
0 & 0 & \mathrm{I}_{n-2}
\end{array}\right) \text {. }
$$

As formas canônicas de Jordan de $\varphi_{1} \circ \varphi_{2}$ e $\left(\varphi_{1} \circ \varphi_{2}\right)^{m}$ são

$$
\left(\begin{array}{cccc}
1 & 1 & 0 & \\
0 & 1 & \vdots & \\
0 & \ldots & 0 & \mathrm{I}_{n-2}
\end{array}\right), \quad\left(\begin{array}{ccc}
1 & m & 0 \\
0 & 1 & 0 \\
0 & 0 & \mathrm{I}_{n-2}
\end{array}\right) .
$$

Portanto, $\psi_{1} \circ \psi_{2}$ não tem ordem finita e $\Delta \cong D_{\infty}$.

Em resumo, seja $\left(\varphi_{1}, \varphi_{2}\right)$ par de involuções lineares transversais em $\mathbb{R}^{2} \operatorname{com} \operatorname{codim} \mathcal{F}\left(\varphi_{1}\right)=$ $\operatorname{codim} \mathcal{F}\left(\varphi_{2}\right)=1$ cuja órbita é representada esquematicamente por um ponto na Figura 2.2. Então, da discussão acima, segue que $\Delta=\left\langle\varphi_{1}, \varphi_{2}\right\rangle \cong D_{\infty}$, exceto se

- $\left(\varphi_{1}, \varphi_{2}\right)$ tem como representante a origem $(0,0)$.

- $\left(\varphi_{1}, \varphi_{2}\right)$ tem como representante o ponto $\left(2+\operatorname{tr}\left(\varphi_{1} \circ \varphi_{2}\right), 1\right)$ onde

$$
\theta=\arccos \operatorname{tr}\left(\frac{\varphi_{1} \circ \varphi_{2}}{2}\right)
$$

com $0<\theta \leq \pi$ e $\theta$ é múltiplo racional de $\pi$. Neste caso, dizemos que $\Delta=D_{m}$ se $m \pi$ é múltiplo de $2 k \pi$, para algum $k$ inteiro.

\subsection{A teoria reversível equivariante}

Num sistema dinâmico podem ocorrer a presença de simetrias e antisimetriass. Quando isto ocorre o sistema é chamado de reversível equivariante. A noção da presença de simetrias e anti-simetrias em equações diferenciais consiste de transformações nas variáveis de estado que preservam o retrato de fase. Enquanto simetrias levam trajetórias em trajetórias, preservando a direção com o tempo, anti-simetrias levam trajetórias em trajetórias revertendo a direção com o tempo. Esta seção tem o propósito de apresentar explicitamente exemplos da teoria reversível equivariante, no caso que o grupo é gerado por pares de involuções nas condições do Teorema 2.3 . 


\subsubsection{Teoria de grupos}

Definição 4.2: Um grupo de Lie linear é um subgrupo $\Gamma$ fechado de $G L(n)$.

Se o grupo $\Gamma$ for compacto, o chamamos apenas Grupo de Lie, pois todo grupo de Lie compacto é topologicamente isomorfo a um grupo de Lie linear (Veja [7]).

Geralmente nos referimos a certos grupos como sendo grupos de Lie, porém são apenas subgrupos isomorfos a algum subgrupo fechado do $G L(n)$. Por exemplo, nos referimos ao grupo multiplicativo $\mathbb{Z}_{2}=\{-1,1\}$ como grupo de Lie, já que existe um isomorfismo com o grupo $\{-\mathrm{I}, \mathrm{I}\} \subset G L(n)$, onde I é a matriz identidade $n \times n$.

Definição 4.3: Seja $\Gamma$ um grupo de Lie e $V$ um espaço vetorial de dimensão finita. Dizemos que $\Gamma$ age linearmente (ou simplesmente age) em $V$ se existe uma aplicação contínua $\Gamma \times V \rightarrow V,(\gamma, v) \mapsto \gamma \cdot v$, tal que:

a) Para cada $\gamma \in \Gamma$, a aplicação $\rho_{\gamma}: V \rightarrow V$ definida por $\rho_{\gamma} v=\gamma \cdot v$ é linear e invertível;

b) Se $\gamma_{1}, \gamma_{2} \in \Gamma$, então $\gamma_{1} \cdot\left(\gamma_{2} \cdot v\right)=\left(\gamma_{1} \gamma_{2}\right) \cdot v$ para todo $v \in V$.

\subsubsection{Germes de funções invariantes e de aplicações equivariantes}

Aqui damos uma descrição de germes de aplicações que comutan com a ação de grupo de Lie compacto $\Gamma$. Começamos com a teoria $\Gamma$-invariante.

Definição 4.4: Seja $\Gamma$ um grupo de Lie compacto agindo num espaço vetorial $V$. Dizemos que um germe de função $f:(V, 0) \rightarrow \mathbb{R}$ é $\Gamma$-invariante se

$$
f(\gamma x)=f(x)
$$

para todo $\gamma \in \Gamma$ e $x \in(V, 0)$.

De forma análoga definimos polinômios de $V \rightarrow \mathbb{R}, \Gamma$-invariantes.

Observação 4.5: No caso em que $\Gamma$ é um grupo finitamente gerado, pela linearidade da ação de $\Gamma$ é suficiente verificar a igualdade (4.1) para o grupo $\Gamma$ apenas para os geradores de $\Gamma$.

Existe um subconjunto finito de polinômios $\Gamma$-invariantes $u_{1}, \ldots, u_{s}$ tal que todo polinômio $\Gamma$-invariante pode ser escrito em função de $u_{1}, \ldots, u_{s}$. Este conjunto, o qual não é único, é chamado de conjunto gerador dos polinômios invariantes, ou base de Hilbert 
Se $\Gamma$ age num espaço $V$, denotamos por $\mathcal{P}_{V}(\Gamma)$ o anel dos polinômios $\Gamma$ - invariantes e por $\mathcal{E}_{V}(\Gamma)$ o anel dos germes de funções $(V, 0) \rightarrow \mathbb{R} \Gamma$ - invariantes. É fácil ver que $\mathcal{P}_{V}(\Gamma)$ e $\mathcal{E}_{V}(\Gamma)$ são de fato anéis sobre $\mathbb{R}$, pois soma e produto de invariantes ainda é um invariante. Se $u_{1}, \ldots, u_{s}$ é uma base de Hilbert para $\mathcal{P}_{V}(\Gamma)$ denotamos $\mathcal{P}_{V}(\Gamma)=\left\langle u_{1}, \ldots, u_{s}\right\rangle$.

Teorema 4.6: Seja $\Gamma$ um grupo de Lie compacto agindo em $V$. Então existem $u_{1}, \ldots, u_{s} \in$ $\mathcal{P}_{V}(\Gamma)$ que geram o anel $\mathcal{P}_{V}(\Gamma)$.

Definição 4.7: Um germe de aplicação $g:(V, 0) \rightarrow(V, 0)$ comuta com $\Gamma$, ou é $\Gamma$ equivariante, se

$$
g(\gamma v)=\gamma g(v), \forall \gamma \in \Gamma, v \in(V, 0)
$$

Se $\Gamma$ age num espaço $V$, denotamos por $\overrightarrow{\mathcal{P}}_{V}(\Gamma)$ o módulo das aplicações polinomiais $\Gamma$ - equivariantes e por $\overrightarrow{\mathcal{E}}_{V}(\Gamma)$ o módulo dos germes de aplicações $\Gamma$-equivariantes.

Teorema 4.8: (Veja [13])Seja $\Gamma$ um grupo de Lie compacto agindo nos espaços $V$ e $W$.

a) O módulo $\overrightarrow{\mathcal{P}}_{V, W}(\Gamma)$ é finitamente gerado sobre o anel $\mathcal{P}_{V}(\Gamma)$.

b) Sejam $g_{1}, \ldots, g_{r}$ geradores para o módulo $\overrightarrow{\mathcal{P}}_{V, W}(\Gamma)$ sobre o anel $\mathcal{P}_{V}(\Gamma)$. Então $g_{1}, \ldots, g_{r}$ também geram o módulo $\overrightarrow{\mathcal{E}}_{V, W}(\Gamma)$ sobre o anel $\mathcal{E}_{V}(\Gamma)$.

Definição 4.9: Dizemos que $g_{1}, \ldots, g_{r}$ geram livremente o módulo $\overrightarrow{\mathcal{E}}_{V}(\Gamma)$ sobre o anel $\mathcal{E}_{V}(\Gamma)$ se a relação $f_{1} g_{1}+\ldots+f_{r} g_{r} \equiv 0, \operatorname{com} f_{j} \in \mathcal{E}_{V}(\Gamma)$ implica $f_{1} \equiv \ldots \equiv f_{r} \equiv 0$.

Neste caso dizemos que $\overrightarrow{\mathcal{E}}_{V}(\Gamma)$ é um módulo livre sobre $\mathcal{E}_{V}(\Gamma)$.

De forma análoga definimos para $\overrightarrow{\mathcal{P}}_{V}(\Gamma)$. Se $g_{1}, \ldots, g_{r}$ geram livremente o módulo $\overrightarrow{\mathcal{E}}_{V}(\Gamma)$ sobre $\mathcal{E}_{V}(\Gamma)$ então para todo $g \in \overrightarrow{\mathcal{E}}_{V}(\Gamma)$, existem únicos $f_{j} \in \mathcal{E}_{V}(\Gamma)$ tais que

$$
g=f_{1} g_{1}+\ldots+f_{r} g_{r}
$$

Seja $\Gamma$ um grupo de Lie compacto e considere um homomorfismo

$$
\sigma: \Gamma \rightarrow \mathbb{Z}_{2}=\{-1,1\}
$$

Note que $\sigma$ define uma representação 1-dimensional de $\Gamma$. Se $\sigma$ é não trivial, então $\Gamma_{+}=\operatorname{ker}(\sigma)$ é um subgrupo normal de $\Gamma$ de índice 2 , e o seu complementar $\Gamma_{-}$é um conjunto não trivial. Fixando um $\delta \in \Gamma_{-}$temos a decomposição de $\Gamma$ como união disjunta

$$
\Gamma=\Gamma_{+} \dot{U} \Gamma_{-}=\Gamma_{+} \dot{U} \delta \Gamma_{+}
$$


Neste caso, o grupo $\Gamma$ é chamado de grupo de simetrias e anti-simetrias. Se $\gamma \in \Gamma_{+}$ dizemos que $\gamma$ é uma simetria em $\Gamma$, enquanto que um elemento de $\Gamma_{-}$é chamado de anti-simetria ou simetria reversível.

Note que o produto entre duas simetrias e entre duas anti-simetrias é uma simetria. Por outro lado, o produto de uma simetria com uma anti-simetria é uma anti-simetria. Observe ainda que o anel $\mathcal{P}_{V}\left(\Gamma_{+}\right)$tem estrutura de módulo sobre o anel $\mathcal{P}_{V}(\Gamma)$.

Definição 4.10: Um germe de função $\tilde{f}:(V, 0) \rightarrow \mathbb{R}$ é chamado de $\Gamma$-anti-invariante se

$$
\tilde{f}(\gamma(x))=\sigma(\gamma) \tilde{f}(x), \forall \gamma \in \Gamma, \forall x \in(V, 0)
$$

Denotamos por $\mathcal{F}_{V}(\Gamma)$ o espaço dos germes de funções $\tilde{f}:(V, 0) \rightarrow \mathbb{R} \Gamma$-anti-invariantes e por $\mathcal{Q}_{V}(\Gamma)$ o espaço de todas as funções polinomiais $\Gamma$-anti-invariantes, os quais têm estrutura de módulo sobre os anéis $\mathcal{E}_{V}(\Gamma)$ e $\mathcal{P}_{V}(\Gamma)$, respectivemente.

Definição 4.11: Um germe de aplicação $\tilde{g}:(V, 0) \rightarrow(V, 0)$ é chamado $\Gamma$-reversívelequivariante se

$$
\tilde{g}(\gamma x)=\sigma(\gamma) \gamma \tilde{g}(x), \forall \gamma \in \Gamma, \forall x \in(V, 0)
$$

Denotamos por $\overrightarrow{\mathcal{F}}_{V}(\Gamma)$ o espaço de todos os germes de aplicações $g:(V, 0) \rightarrow(V, 0)$ $\Gamma$-equivariantes e por $\overrightarrow{\mathcal{Q}}_{V}(\Gamma)$ o espaço de todas as aplicações polinomiais $\Gamma$-reversíveisequivariantes, os quais têm estrutura de módulos sobre os anéis $\mathcal{E}_{V}(\Gamma)$ e $\mathcal{P}_{V}(\Gamma)$, respectivemente.

\subsection{Grupos conjugados e a teoria reversível equivariante}

Nesta seção exibimos a relação entre os conjuntos $\mathcal{P}, \overrightarrow{\mathcal{P}}, \mathcal{Q}$ e $\overrightarrow{\mathcal{Q}}$ para grupos com representações conjugadas por um isomorfismo linear.

Seja $\Gamma=\left\langle\varphi_{1}, \varphi_{2}\right\rangle$ o grupo gerado por um par $\left(\varphi_{1}, \varphi_{2}\right)$ de involuções lineares transversais no $\mathbb{R}^{n}$ e seja $\widetilde{\Gamma}$ um grupo conjugado a $\Gamma$ por um isomorfismo linear $h$ isto é $\widetilde{\Gamma}=\left\{h \circ \gamma \circ h^{-1} ; \gamma \in \Gamma\right\}$.

Consideremos o homomorfismo de grupos (4.3).Agora seja o homomorfismo $h^{*}: \Gamma \longrightarrow$ $\widetilde{\Gamma}$ onde $h^{*}(\gamma)=h \circ \gamma \circ h^{-1}$. Desde que $\Gamma$ tem simetrias e anti-simetrias consideremos um homomorfismo natural para $\widetilde{\Gamma}$,

$$
\begin{aligned}
\widetilde{\sigma}: \widetilde{\Gamma} & \rightarrow \mathbb{Z}_{2} \\
\widetilde{\gamma} & \rightarrow \widetilde{\sigma}(\widetilde{\gamma})=\sigma \circ\left(h^{*}\right)^{-1}(\widetilde{\gamma}) .
\end{aligned}
$$


Por construção, o homomorfismo $h^{*}$ leva simetrias de $\Gamma$ em simetrias de $\widetilde{\Gamma}$ e anti-simetrias de $\Gamma$ em anti-simetrias de $\widetilde{\Gamma}$, ou seja $\sigma(\gamma)=\widetilde{\sigma}(\widetilde{\gamma})$ para $\widetilde{\gamma}=h \circ \gamma \circ h^{-1}$.

Proposição 4.12: $\quad$ (a) $p: \mathbb{R}^{n} \rightarrow \mathbb{R}$ é $\Gamma$-invariante se, e só se, $p \circ h^{-1}$ é $\widetilde{\Gamma}$-invariante.

(b) $f: \mathbb{R}^{n} \rightarrow \mathbb{R}^{n}$ é $\Gamma$-equivariante se, e só se, $h \circ f \circ h^{-1}$ é $\widetilde{\Gamma}$-equivariante.

(c) $p: \mathbb{R}^{n} \rightarrow \mathbb{R}$ é $\Gamma$ anti-invariante se, e só se, $p \circ h^{-1}$ é $\widetilde{\Gamma}$ anti-invariante.

(d) $f: \mathbb{R}^{n} \rightarrow \mathbb{R}^{n}$ é $\Gamma$ reversível-equivariante se, e só se, $h \circ f \circ h^{-1}$ é $\widetilde{\Gamma}$ reversívelequivariante.

\section{Demonstração:}

(a) Suponha $p \circ h^{-1} \widetilde{\Gamma}$-invariante, então $p(\gamma)=p\left(h^{-1} \circ \widetilde{\gamma} \circ h\right)=p \circ h^{-1}(\widetilde{\gamma} \circ h)=p$. Reciprocamente, se $p$ é $\Gamma$-invariante, então $p \circ h^{-1}(\widetilde{\gamma})=p \circ h^{-1}\left(h \circ \gamma \circ h^{-1}\right)=p \circ h^{-1}$. Portanto,

$$
\mathcal{P}(\widetilde{\Gamma})=\left\{p \circ h^{-1}: p \in \mathcal{P}(\Gamma)\right\}
$$

(b) Suponha $h \circ f \circ h^{-1} \widetilde{\Gamma}$-equivariante, então $f(\gamma)=f\left(h^{-1} \circ \widetilde{\gamma} \circ h\right)=h^{-1} \circ h \circ f \circ h^{-1}(\widetilde{\gamma} \circ$ $h)=h^{-1} \circ \widetilde{\gamma} \circ h \circ f \circ h^{-1} \circ h=\gamma \circ f$. Reciprocamente, se $f$ é $\Gamma$-equivariante, então $h \circ f \circ h^{-1}(\widetilde{\gamma})=h \circ f \circ h^{-1}\left(h \circ \gamma \circ h^{-1}\right)=h \circ \gamma \circ f \circ h^{-1}=\widetilde{\gamma}\left(h \circ f \circ h^{-1}\right)$ Portanto,

$$
\overrightarrow{\mathcal{P}}(\widetilde{\Gamma})=\left\{h \circ f \circ h^{-1}: f \in \overrightarrow{\mathcal{P}}(\Gamma)\right\}
$$

(c) Suponha $p \circ h^{-1} \widetilde{\Gamma}$-anti-invariante, então $p(\gamma)=p\left(h^{-1} \circ \widetilde{\gamma} \circ h\right)=p \circ h^{-1}(\widetilde{\gamma} \circ h)=\widetilde{\sigma}(\widetilde{\gamma}) p$. Reciprocamente, se $p$ é $\Gamma$-invariante, então $p \circ h^{-1}(\widetilde{\gamma})=p \circ h^{-1}\left(h \circ \gamma \circ h^{-1}\right)=$ $\sigma(\gamma) p \circ h^{-1}=\widetilde{\sigma}(\widetilde{\gamma}) p \circ h^{-1}$. Portanto,

$$
\mathcal{Q}(\widetilde{\Gamma})=\left\{p \circ h^{-1}: p \in \mathcal{Q}(\Gamma)\right\}
$$

(d) Suponha $h \circ f \circ h^{-1} \widetilde{\Gamma}$-reversível-equivariante, então $f(\gamma)=f\left(h^{-1} \circ \widetilde{\gamma} \circ h\right)=h^{-1} \circ$ $h \circ f \circ h^{-1}(\widetilde{\gamma} \circ h)=h^{-1} \circ \tilde{\sigma}(\widetilde{\gamma}) \widetilde{\gamma} \circ h \circ f \circ h^{-1} \circ h=\widetilde{\sigma}(\widetilde{\gamma}) h^{-1} \circ \widetilde{\gamma} \circ h \circ f=\sigma(\gamma) \gamma \circ f$. Reciprocamente, se $f \Gamma$-reversível-equivariante, então $h \circ f \circ h^{-1}(\widetilde{\gamma})=h \circ f \circ h^{-1}(h \circ$ $\left.\gamma \circ h^{-1}\right)=h \circ f \circ \gamma \circ h^{-1}=h \circ \sigma(\gamma) \gamma \circ f \circ h^{-1}=\sigma(\gamma) \widetilde{\gamma} \circ h \circ f \circ h^{-1}=\tilde{\sigma}(\widetilde{\gamma}) \widetilde{\gamma} \circ h \circ f \circ h^{-1}$. Portanto,

$$
\overrightarrow{\mathcal{Q}}(\widetilde{\Gamma})=\left\{h \circ f \circ h^{-1}: f \in \overrightarrow{\mathcal{Q}}(\Gamma)\right\}
$$




\subsection{Exemplos}

Nosso interesse está em considerar apenas os grupos $\Gamma=\left\langle\varphi_{1}, \varphi_{2}\right\rangle$ gerados pelo par $\left(\varphi_{1}, \varphi_{2}\right)$ de involuções. Explicitamos a seguir os conjuntos $\mathcal{P}, \overrightarrow{\mathcal{P}}, \mathcal{Q}$ e $\overrightarrow{\mathcal{Q}}$ quando $\Gamma=$ $\left\langle\varphi_{1}, \varphi_{2}\right\rangle$ e o par de involuções está nas condições do Teorema 2.3 .

Seja $\Gamma=\left\langle\varphi_{1}, \varphi_{2}\right\rangle$ agindo em $\mathbb{R}^{2}$, onde $\left(\varphi_{1}, \varphi_{2}\right)$ é um par de involuções lineares transversais. Pelos resultados anteriores sabemos que é suficiente estudar a teoria reversível equivariante para os pares representantes na Figura 2.2.

Exemplos 4.13: Suponha $\varphi_{1}(x, y)=(-x, y)$ e $\varphi_{2}(x, y)=(x,-y)$ e definamos $\Gamma_{+}=$ $\{\mathrm{I},-\mathrm{I}\}$ e $\Gamma_{-}=\left\{\varphi_{1}, \varphi_{2}\right\}$. Neste caso, $\Gamma=\mathbb{Z}_{2} \times \mathbb{Z}_{2}$

- $\mathcal{P}(\Gamma)$ : Calculamos manualmente os elementos $f_{1}(x, y)=x^{2}$ e $f_{2}(x, y)=y^{2}$, os quais formam uma base para o anel $\mathcal{P}\left(\mathbb{Z}_{2} \times \mathbb{Z}_{2}\right)$.

- $\overrightarrow{\mathcal{P}}(\Gamma)$ : Os geradores para o módulo $\overrightarrow{\mathcal{P}}\left(\mathbb{Z}_{2} \times \mathbb{Z}_{2}\right)$ sobre o anel $\mathcal{P}\left(\mathbb{Z}_{2} \times \mathbb{Z}_{2}\right)$ são $u_{1}(x, y)=$ $(x, 0)$ e $u_{2}(x, y)=(0, y)$.

- $\mathcal{Q}(\Gamma)$ : O gerador para o módulo $\mathcal{Q}\left(\mathbb{Z}_{2} \times \mathbb{Z}_{2}\right)$ sobre o anel $\mathcal{P}\left(\mathbb{Z}_{2} \times \mathbb{Z}_{2}\right)$ é $g_{1}(x, y)=x y$.

- $\overrightarrow{\mathcal{Q}}(\Gamma)$ : Os geradores para o módulo $\overrightarrow{\mathcal{Q}}\left(\mathbb{Z}_{2} \times \mathbb{Z}_{2}\right)$ sobre o anel $\mathcal{P}\left(\mathbb{Z}_{2} \times \mathbb{Z}_{2}\right)$ são $v_{1}(x, y)=$ $(y, 0)$ e $v_{2}(x, y)=(0, x)$.

Para o seguinte exemplo denotamos o máximo inteiro de $n$ por $[|n|]$.

Exemplos 4.14: Suponha $\left(\varphi_{1}, \varphi_{2}\right)$ tal que $-2<\operatorname{tr}\left(\varphi_{1} \circ \varphi_{2}\right)<2$. Escolhemos o par $\left(\varphi_{1}, \varphi_{2}\right)$ de forma que sejan reflexões tais que $\varphi_{1}(x, y)=(x,-y)$ e $\varphi_{1} \circ \varphi_{2}=R_{2 k \pi}$, onde $k$ é um racional e definimos $\Gamma_{+}=\left\langle\varphi_{1} \circ \varphi_{2}\right\rangle$ e $\varphi_{1}, \varphi_{2} \in \Gamma_{-}$. Suponha $k=1 / n$.

- $\mathcal{P}(\Gamma)$ : Calculamos os elementos

$$
\begin{gathered}
f_{1}(x, y)=x^{2}+y^{2} \mathrm{e} \\
f_{2}(x, y)=\sum_{l=0}^{[|n / 2|]}(-1)^{l}\left(\begin{array}{c}
n \\
2 l
\end{array}\right) x^{n-2 l} y^{2 l},
\end{gathered}
$$

os quais formam uma base para o anel $\mathcal{P}(\Gamma)$.

- $\overrightarrow{\mathcal{P}}(\Gamma)$ : Os geradores para o módulo $\overrightarrow{\mathcal{P}}(\Gamma)$ sobre o anel $\mathcal{P}(\Gamma)$ são

$$
u_{1}(x, y)=\sum_{l=0}^{[|n-1 / 2|]}(-1)^{l}\left(\begin{array}{c}
n-1 \\
2 l
\end{array}\right) x^{n-2 l-1} y^{2 l},
$$




$$
u_{2}(x, y)=\sum_{l=0}^{[|n-2 / 2|]}(-1)^{l+1}\left(\begin{array}{c}
n-1 \\
2 l+1
\end{array}\right) x^{n-2 l-2} y^{2 l+1}
$$

- $\mathcal{Q}(\Gamma)$ : O gerador para o módulo $\mathcal{Q}(\Gamma)$ sobre o anel $\mathcal{P}(\Gamma)$ é

$$
g_{1}(x, y)=\sum_{l=0}^{[|n-1 / 2|]}(-1)^{l}\left(\begin{array}{c}
n \\
2 l+1
\end{array}\right) x^{n-2 l-1} y^{2 l+1}
$$

- $\overrightarrow{\mathcal{Q}}(\Gamma)$ : Os geradores para o módulo $\overrightarrow{\mathcal{Q}}(\Gamma)$ sobre o anel $\mathcal{P}(\Gamma)$ são:

$$
\begin{gathered}
v_{1}(x, y)=\sum_{l=0}^{[|n-2 / 2|]}(-1)^{l}\left(\begin{array}{c}
n-1 \\
2 l+1
\end{array}\right) x^{n-2 l-2} y^{2 l+1}, \\
v_{2}(x, y)=\sum_{l=0}^{[|n-1 / 2|]}(-1)^{l}\left(\begin{array}{c}
n-1 \\
2 l
\end{array}\right) x^{n-2 l-1} y^{2 l} .
\end{gathered}
$$

Agora suponhamos $k=p / n$, onde $p$ e $n$ são primos entre si, então $\varphi_{1}(x, y)=(x,-y)$ e $\varphi_{1} \circ \varphi_{2}=R_{\frac{2 p \pi}{n}}$. Neste caso $\mathcal{P}, \overrightarrow{\mathcal{P}}, \mathcal{Q}$ e $\overrightarrow{\mathcal{Q}}$ são os mesmos que para o caso $p=1$.

Exemplos 4.15: Suponha $\left(\varphi_{1}, \varphi_{2}\right)$ tal que $-2<\operatorname{tr}\left(\varphi_{1} \circ \varphi_{2}\right)<2$. Então pela Subseção 3.2 , podemos escolher $\varphi_{1}, \varphi_{2}$ como duas reflexões tais que $\varphi_{1}(x, y)=(x,-y)$ e $\varphi_{1} \circ \varphi_{2}=$ $R_{2 k \pi}$, onde $k$ não é racional. Definamos $\Gamma_{+}=\left\langle\varphi_{1} \circ \varphi_{2}\right\rangle$ e $\varphi_{1}, \varphi_{2} \in \Gamma_{-}$.

- $\mathcal{P}(\Gamma)$ : Calculamos $f_{1}(x, y)=x^{2}+y^{2}$ o qual é gerador do anel $\mathcal{P}(\Gamma)$.

- $\overrightarrow{\mathcal{P}}(\Gamma)$ : O gerador para o módulo $\overrightarrow{\mathcal{P}}(\Gamma)$ sobre o anel $\mathcal{P}(\Gamma)$ é $u_{1}(x, y)=(x, y)$.

- $\mathcal{Q}(\Gamma)$ : Neste caso $\mathcal{Q}(\Gamma)=\{0\}$.

- $\overrightarrow{\mathcal{Q}}(\Gamma)$ : O gerador para o módulo $\overrightarrow{\mathcal{Q}}(\Gamma)$ sobre o anel $\mathcal{P}(\Gamma)$ é $v_{1}(x, y)=(-y, x)$.

Exemplos 4.16: Suponha $\varphi_{1}, \varphi_{2}$ tais que $\operatorname{tr}\left(\varphi_{1} \circ \varphi_{2}\right)>2$ ou $\operatorname{tr}\left(\varphi_{1} \circ \varphi_{2}\right)<-2$. Então, pelo Teorema 2.3 podemos escolher $\varphi_{1}, \varphi_{2}$ da seguinte forma:

$$
\varphi_{1}(x, y)=\left(-x, y+\left(2+\operatorname{tr}\left(\varphi_{1} \circ \varphi_{2}\right)\right) x\right), \quad \varphi_{2}(x, y)=(x+y,-y) .
$$

Mas $\left(\varphi_{1}, \varphi_{2}\right)$ é equivalente ao par $\left(\psi_{1}, \psi_{2}\right)$ de involuções lineares transversais

$$
\psi_{1}(x, y)=(-y,-x), \psi_{2}(x, y)=\left(-\lambda^{-1} y,-\lambda x\right)
$$

onde $\lambda, \lambda^{-1}$ são as raízes do polinômio característico de $\varphi_{1} \circ \varphi_{2}$. Assim pelos resultados anteriores é suficiente estudar o caso $\Gamma=\left\langle\psi_{1}, \psi_{2}\right\rangle=\left\langle\psi_{1}, \psi_{1} \circ \psi_{2}\right\rangle$. Observamos que $\psi_{1} \circ \psi_{2}(x, y)=\left(\lambda x, \lambda^{-1} y\right)$ e definimos $\Gamma_{+}=\left\langle\psi_{1} \circ \psi_{2}\right\rangle$ e $\psi_{1}, \psi_{2} \in \Gamma_{-}$. 
- $\mathcal{P}(\Gamma)$ : Calculamos o gerador do anel $\mathcal{P}(\Gamma)$ que é $f_{1}(x, y)=x y$.

- $\overrightarrow{\mathcal{P}}(\Gamma)$ : O gerador para o módulo $\overrightarrow{\mathcal{P}}(\Gamma)$ sobre o anel $\mathcal{P}(\Gamma)$ é $f_{1}(x, y)=(x, y)$.

- $\mathcal{Q}(\Gamma)$ : Neste caso $\mathcal{Q}(\Gamma)=\{0\}$.

- $\overrightarrow{\mathcal{Q}}(\Gamma)$ : O gerador para o módulo $\overrightarrow{\mathcal{Q}}(\Gamma)$ sobre o anel $\mathcal{P}(\Gamma)$ é $v_{1}(x, y)=(x,-y)$.

Exemplos 4.17: Suponha que o par $\left(\varphi_{1}, \varphi_{2}\right)$ é representado pelo ponto $(1,0)$ na Figura 2.2. Então podemos escolher $\left(\varphi_{1}, \varphi_{2}\right)$ como $\varphi_{1}(x, y)=(-x, x+y)$ e $\varphi_{2}(x, y)=(x,-y) \mathrm{e}$ definimos $\Gamma_{+}=\left\langle\varphi_{1} \circ \varphi_{2}\right\rangle$ e $\psi_{1}, \psi_{2} \in \Gamma_{-}$. Então,

- $\mathcal{P}(\Gamma)$ : Calculamos o gerador do anel $\mathcal{P}(\Gamma)$ que é $f_{1}(x, y)=x^{2}$.

- $\overrightarrow{\mathcal{P}}(\Gamma)$ : O gerador para o módulo $\overrightarrow{\mathcal{P}}(\Gamma)$ sobre o anel $\mathcal{P}(\Gamma)$ é $f_{1}(x, y)=(x, y)$.

- $\mathcal{Q}(\Gamma)$ : Neste caso $\mathcal{Q}(\Gamma)=\{0\}$.

- $\overrightarrow{\mathcal{Q}}(\Gamma)$ : O gerador para o módulo $\overrightarrow{\mathcal{Q}}(\Gamma)$ sobre o anel $\mathcal{P}(\Gamma)$ é $v_{1}(x, y)=(0,-x)$.

Exemplos 4.18: Suponha $\varphi_{1}(x, y)=(-x, y)$ e $\varphi_{2}(x, y)=(x+y,-y)$ ou seja $\operatorname{tr}\left(\varphi_{1} \circ\right.$ $\left.\varphi_{2}\right)=-2$ e definimos $\Gamma_{+}=\left\langle\varphi_{1} \circ \varphi_{2}\right\rangle$ e $\psi_{1}, \psi_{2} \in \Gamma_{-}$. Então

- $\mathcal{P}(\Gamma)$ Calculamos o gerador do anel $\mathcal{P}(\Gamma)$ que é $f_{1}(x, y)=y^{2}$.

- $\overrightarrow{\mathcal{P}}(\Gamma)$ O gerador para o módulo $\overrightarrow{\mathcal{P}}(\Gamma)$ sobre o anel $\mathcal{P}(\Gamma)$ é $f_{1}(x, y)=(x, y)$.

- $\mathcal{Q}(\Gamma)$ : Neste caso $\mathcal{Q}(\Gamma)=\{0\}$.

- $\overrightarrow{\mathcal{Q}}(\Gamma)$ O gerador para o módulo $\overrightarrow{\mathcal{Q}}(\Gamma)$ sobre o anel $\mathcal{P}(\Gamma)$ é $v_{1}(x, y)=(y, 0)$.

Exemplos 4.19: Suponha $\varphi_{1}(x, y)=(-x, 4 x+y)$ e $\varphi_{2}(x, y)=(x+y,-y)$. Notemos que este é o caso onde $\operatorname{tr}\left(\varphi_{1} \circ \varphi_{2}\right)=2$. Então,

- $\mathcal{P}(\Gamma)$ Calculamos o gerador do anel $\mathcal{P}(\Gamma)$ que é $f_{1}(x, y)=x$.

- $\overrightarrow{\mathcal{P}}(\Gamma)$ O gerador para o módulo $\overrightarrow{\mathcal{P}}(\Gamma)$ sobre o anel $\mathcal{P}(\Gamma)$ é $f_{1}(x, y)=(x, y)$.

- $\mathcal{Q}(\Gamma)$ : Neste caso $\mathcal{Q}(\Gamma)=\{0\}$.

- $\overrightarrow{\mathcal{Q}}(\Gamma)$ O gerador para o módulo $\overrightarrow{\mathcal{Q}}(\Gamma)$ sobre o anel $\mathcal{P}(\Gamma)$ é $v_{1}(x, y)=(0,0)$.

Com base na Proposição 4.12 , observamos finalmente que, se tomamos un par de involuções lineares tranversais qualquer, para encontrar $\mathcal{P}, \overrightarrow{\mathcal{P}}, \mathcal{Q}$ e $\overrightarrow{\mathcal{Q}}$ é suficiente encontrar a conjugação entre este par e o par correspondente da Figura 2.2 e aplicar os resultados acima. 
52 Capítulo 4. Grupos gerados por um par de involuções e a teoria reversível equivariante 


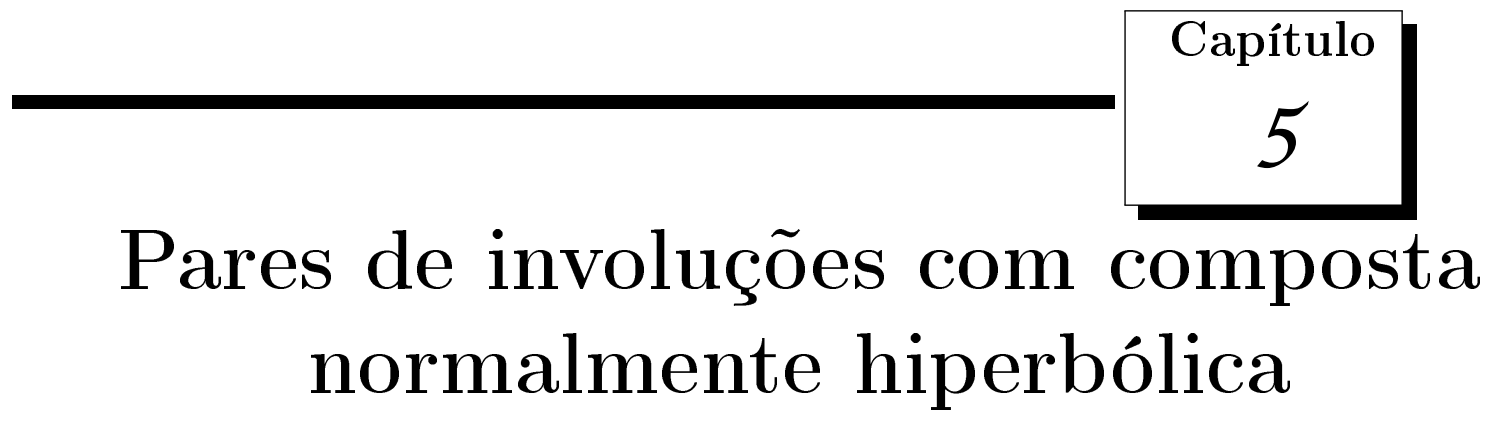

O comportamento silmultâneo de difeomorfismos tem sido estudado en diferentes trabalhos com obtenção de resultados importantes na teoria de singularidades e sistemas dinânicos. Mencionamos por exemplo o Teorema de Bochner-Montgomery (veja [12]), que é um resultado sobre linearização de um grupo compacto de aplicações em torno de um ponto. Neste capítulo abordamos o estudo de uma classe de pares $\left(\varphi_{1}, \varphi_{2}\right)$ de involuções com composta normalmente hiperbólica onde usamos como referência básica [11]. Mencionamos um teorema de linearização simultânea destes pares de involuções. E como conseqüência, fazemos um estudo quando a composta é hiperbólica.

Apresentamos formas normais para dimensões explícitas dentro dos casos hiperbólico e normalmente hiperbólico. E finalmente, com a hipótese de hiperbolicidade normal, encontramos uma relação entre os conjuntos $\mathcal{F}\left(\varphi_{1} \circ \varphi_{2}\right)$ e $\mathcal{F}\left(\varphi_{1}\right), \mathcal{F}\left(\varphi_{2}\right)$.

\subsection{Um teorema de linearização}

Como já temos visto no Capítulo 1 , dada uma involução em $\left(\mathbb{R}^{n}, 0\right)$, o germe de difeomorfismo $h=\frac{1}{2}(\mathrm{I}+d \varphi(0) \circ \varphi)$ de $\left(\mathbb{R}^{n}, 0\right)$ é uma conjugação entre $\varphi$ e o germe da sua parte linear $d \varphi(0)$ no 0 , isto é

$$
d \varphi(0)=h \circ \varphi \circ h^{-1}
$$

De fato,

$$
d \varphi(0) \circ h=\frac{1}{2} d \varphi(0)(\mathrm{I}+d \varphi(0) \circ \varphi)=\frac{1}{2}(d \varphi(0)+\varphi)=\frac{1}{2}(\mathrm{I}+d \varphi(0) \circ \varphi) \varphi=h \circ \varphi .
$$


Definição 5.1: Seja $f:\left(\mathbb{R}^{n}, 0\right) \rightarrow\left(\mathbb{R}^{n}, 0\right)$ um germe de difeomorfismo, $f \neq \mathrm{I}$. Suponha que $\mathcal{F}(f)$ é uma subvariedade em $\left(\mathbb{R}^{n}, 0\right)$ e que $\operatorname{dim} \mathcal{F}(f)=k$. Dizemos que $f$ é normalmente hiperbólico se o espectro de $d f(0)$ tem, contando multiplicidade, $n-k$ elementos fora do círculo unitário $S^{1} \subset \mathbb{C}$.

Notemos que se $\mathcal{F}(f)=\{0\}$ a definição acima se reduz ao conceito de germe de difeomorfismo hiperbólico. Se $\operatorname{dim} \mathcal{F}(f)=k>0$ então, 1 é autovalor de $d f(0)$ com multiplicidade algébrica e geométrica iguais a $k$, pois

$$
T_{0} \mathcal{F}(f)=\mathcal{F}(d f(0)),
$$

e $\operatorname{dim} \mathcal{F}(f)=\operatorname{dim} T_{0} \mathcal{F}(f)=\operatorname{dim} \mathcal{F}(d f(0))=k$. Logo, $d f(0)$ é também um isomorfismo normalmente hiperbólico.

Lembrando da definição de transversalidade de $s$-uplas de involuções no Capítulo 1, Definição 1.15, para o caso particular $s=2$ temos que: Duas involuções $\varphi_{1}, \varphi_{2}:\left(\mathbb{R}^{n}, 0\right) \rightarrow$ $\left(\mathbb{R}^{n}, 0\right)$ são transversais se $\mathcal{F}\left(\varphi_{1}\right)$ e $\mathcal{F}\left(\varphi_{2}\right)$ estão em posição geral no 0 , isto é

$$
\mathbb{R}^{n}=T_{0} \mathcal{F}\left(\varphi_{1}\right)+T_{0} \mathcal{F}\left(\varphi_{2}\right)
$$

Com a transversalidade de involuções observamos que a menos de equivalência, não perdemos a generalidade em assumir que o par satisfaz

$$
\mathcal{F}\left(\varphi_{i}\right)=\mathcal{F}\left(d \varphi_{i}(0)\right), i=1,2
$$

De fato, sejam $\varphi_{1}, \varphi_{2}$ transversais, pelo Lema 3.10 em [7] (linearização simultânea de subvariedades transversais) podemos tomar o germe de difeomorfismo $h$ de $\left(\mathbb{R}^{n}, 0\right)$ tal que $h\left(\mathcal{F}\left(\varphi_{1}\right)\right)$ e $h\left(\mathcal{F}\left(\varphi_{2}\right)\right)$ são subvariedades lineares, isto é, $h$ lineariza simultaneamente as subvariedades $\mathcal{F}\left(\varphi_{1}\right)$ e $\mathcal{F}\left(\varphi_{2}\right)$. Consideremos o par de involuções $\left(\psi_{1}, \psi_{2}\right)$ tais que

$$
\psi_{i}=h \circ \varphi_{i} \circ h^{-1}, i=1,2 .
$$

Então, $\left(\varphi_{1}, \varphi_{2}\right)$ é equivalente a $\left(\psi_{1}, \psi_{2}\right)$ onde para este par temos que

$$
\mathcal{F}\left(\psi_{i}\right)=\mathcal{F}\left(d \psi_{i}(0)\right), i=1,2
$$

em $\left(\mathbb{R}^{n}, 0\right)$.

Ainda para a descrição da estrutura da classe de pares de involuções para as quais se aplica o teorema de linearização, consideramos $L: \mathbb{R}^{n} \rightarrow \mathbb{R}^{n}$ um isomorfismo linear normalmente hiperbólico e tomamos a descomposição

$$
\mathbb{R}^{n}=E^{s} \oplus E^{u} \oplus \mathcal{F}(L)
$$


onde $E^{s}$ e $E^{u}$ são os subespaços estável e instável de $L$ respectivamente. Seja

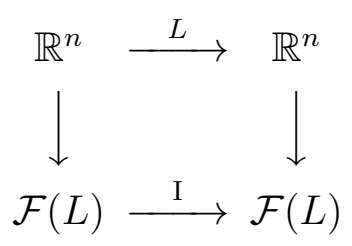

o automorfismo fibrado hiperbólico cobrindo a identidade I, cujas fibras são iguais a $E^{s} \oplus E^{u}$. Agora estamos em posição de enunciar o teorema:

Teorema 5.2: $\quad$ Seja $\left(\varphi_{1}, \varphi_{2}\right)$ um par de involuçôes transversais em $\left(\mathbb{R}^{n}, 0\right)$ tal que $\mathcal{F}\left(\varphi_{i}\right)=$ $\mathcal{F}\left(d \varphi_{i}(0)\right), i=1,2, \varphi_{1} \circ \varphi_{2}$ é normalmente hiperbólico e localmente cada $\varphi_{i}$ respeita o fibrado em (5.3) para $L=d\left(\varphi_{1} \circ \varphi_{2}\right)(0)$. Então, este par é $C^{0}$-equivalente a $\left(L_{1}, L_{2}\right)$, onde $L_{i}=d \varphi_{i}(0), i=1,2$.

Este é o principal resultado de [11]. Com base neste teorema, passa a ser de interesse o conhecimento das classes de pares de involuções lineares transversais com composta normalmente hiperbólica. É disto que trata a subseção seguinte. 


\subsection{Classificação de pares de involuções lineares trans- versais com composta normalmente hiperbólica}

Nesta seção apresentamos a classificação de pares de involuções lineares com composta normalmente hiperbólica para o caso onde os conjuntos dos pontos fixos são transversais. Esta classificação é dada por conjugação linear e as formas normais são apresentadas nos Teoremas 5.11 e 5.12 .

\subsubsection{Primeiros resultados sobre hiperbolicidade}

Começamos com dois resultados com respeito à composição de duas involuções lineares que são essenciais para tudo a seguir. Lembremos que para uma involução linear $\varphi$ em $\mathbb{R}^{n}$, temos a seguinte descomposição

$$
\mathbb{R}^{n}=\mathcal{F}(\varphi) \oplus \mathcal{A}(\varphi)
$$

Proposição 5.3: Sejam $\varphi_{1}, \varphi_{2}$ involuções lineares em $\mathbb{R}^{n}$. Verifica-se os seguintes resultados:

(a) $\mathcal{F}\left(\varphi_{1} \circ \varphi_{2}\right)=\mathcal{F}\left(\varphi_{1}\right) \cap \mathcal{F}\left(\varphi_{2}\right) \oplus \mathcal{A}\left(\varphi_{1}\right) \cap \mathcal{A}\left(\varphi_{2}\right)$,

(b) $\mathcal{A}\left(\varphi_{1} \circ \varphi_{2}\right)=\mathcal{F}\left(\varphi_{1}\right) \cap \mathcal{A}\left(\varphi_{2}\right) \oplus \mathcal{A}\left(\varphi_{1}\right) \cap \mathcal{F}\left(\varphi_{2}\right)$.

Demonstração: Dado $x \in \mathbb{R}^{n}$, podemos escrever

$$
x=\frac{x+\varphi_{i}(x)}{2}+\frac{x-\varphi_{i}(x)}{2},
$$

de modo que $x$ é uma soma de elementos de $\mathcal{F}\left(\varphi_{i}\right)$ e $\mathcal{A}\left(\varphi_{i}\right)$ como em (5.4). Agora vejamos que

$$
x \in \mathcal{F}\left(\varphi_{1} \circ \varphi_{2}\right) \Leftrightarrow \varphi_{1}(x)=\varphi_{2}(x) \Leftrightarrow \frac{x \pm \varphi_{1}(x)}{2}=\frac{x \pm \varphi_{2}(x)}{2} .
$$

Isto prova $(\subseteq)$ no item $(a)$, o outro lado é trivial. Para provar a parte $(b)$ basta observar que

$$
x \in \mathcal{A}\left(\varphi_{1} \circ \varphi_{2}\right) \Leftrightarrow \varphi_{1}(x)=-\varphi_{2}(x) \Leftrightarrow \frac{x \pm \varphi_{1}(x)}{2}=\frac{x \mp \varphi_{2}(x)}{2} .
$$

Corolário 5.4: Sejam $\varphi_{1}, \varphi_{2}$ involuções lineares em $\mathbb{R}^{n}$. Se $\mathcal{A}\left(\varphi_{1} \circ \varphi_{2}\right)=\{0\}$, então $\operatorname{dim} \mathcal{F}\left(\varphi_{1}\right)=\operatorname{dim} \mathcal{F}\left(\varphi_{2}\right)$. 
Demonstração: Suponha $\mathcal{A}\left(\varphi_{1} \circ \varphi_{2}\right)=\{0\}$. Pela Proposição 5.3 temos que $\mathcal{F}\left(\varphi_{1}\right) \cap$ $\mathcal{A}\left(\varphi_{2}\right)=\{0\}$ e $\mathcal{A}\left(\varphi_{1}\right) \cap \mathcal{F}\left(\varphi_{2}\right)=\{0\}$. Portanto,

$$
\operatorname{dim} \mathcal{F}\left(\varphi_{1}\right)+\left(n-\operatorname{dim} \mathcal{F}\left(\varphi_{2}\right)\right)=\operatorname{dim} \mathcal{F}\left(\varphi_{1}\right)+\operatorname{dim} \mathcal{A}\left(\varphi_{2}\right)=\operatorname{dim}\left(\mathcal{F}\left(\varphi_{1}\right)+\mathcal{A}\left(\varphi_{2}\right)\right) \leq n
$$

o que implica que $\operatorname{dim} \mathcal{F}\left(\varphi_{1}\right) \leq \operatorname{dim} \mathcal{F}\left(\varphi_{2}\right)$. Analogamente conseguimos que $\operatorname{dim} \mathcal{F}\left(\varphi_{2}\right) \leq$ $\operatorname{dim} \mathcal{F}\left(\varphi_{1}\right)$ fazendo um intercâmbio entre $\varphi_{1}$ e $\varphi_{2}$.

Observação 5.5: Dois pares lineares de involuções equivalentes $\left(\varphi_{1}, \varphi_{2}\right)$ e $\left(\psi_{1}, \psi_{2}\right)$ são linearmente equivalentes, digamos por $h$. Temos então $h\left(\mathcal{F}\left(\varphi_{i}\right)\right)=\mathcal{F}\left(\psi_{i}\right)$ e também

$$
h\left(\mathcal{A}\left(\varphi_{i}\right)\right)=\mathcal{A}\left(\psi_{i}\right), i=1,2 .
$$

Ainda mais, dois pares de involuções lineares $\left(\varphi_{1}, \varphi_{2}\right)$ e $\left(\psi_{1}, \psi_{2}\right)$ em $\mathbb{R}^{n}$ são linearmente equivalentes se, e só se, $\left(-\varphi_{1},-\varphi_{2}\right)$ e $\left(-\psi_{1},-\psi_{2}\right)$ são linearmente equivalentes. De fato, é imediato que

$$
\psi_{i}=h \circ\left(\varphi_{i}\right) \circ h^{-1} \text { se, e só se, }-\psi_{i}=h \circ\left(-\varphi_{i}\right) \circ h^{-1},
$$

já que $h$ é isomorfismo linear.

Para duas involuções $\varphi_{1}, \varphi_{2}$ lineares a transversalidade (5.1) fica

$$
\mathcal{F}\left(\varphi_{1}\right)+\mathcal{F}\left(\varphi_{2}\right)=\mathbb{R}^{n}
$$

Observação 5.6: Consideremos $\varphi_{1}$ e $\varphi_{2}$ involuções lineares e a composição $\varphi_{1} \circ \varphi_{2}$ normalmente hiperbólica. Note que se $\varphi_{1}$ e $\varphi_{2}$ não são transversais mas $\mathcal{A}\left(\varphi_{1}\right)$ e $\mathcal{A}\left(\varphi_{2}\right)$ estão em posição geral, então, é possível obter a forma normal do par $\left(\varphi_{1}, \varphi_{2}\right)$ aplicando os resultados a $\left(-\varphi_{1},-\varphi_{2}\right)$. Isto é conseqüência da Observação 5.5 e do fato que

$$
\mathcal{F}\left(-\varphi_{i}\right)=\mathcal{A}\left(\varphi_{i}\right)
$$

Em algumas dimensões, isto dá a classificação completa de pares de involuções lineares com composição normalmente hiperbólica. Estes são precisamente os casos para os quais a hiperbolicidade normal implica que ou $\mathcal{F}\left(\varphi_{1}\right)$ e $\mathcal{F}\left(\varphi_{2}\right)$ ou $\mathcal{A}\left(\varphi_{1}\right)$ e $\mathcal{A}\left(\varphi_{2}\right)$ estão em posição geral.

\subsubsection{A classificação}

Seja $\left(\varphi_{1}, \varphi_{2}\right)$ um par de involuções lineares em $\mathbb{R}^{n}, n \geq 2$, tal que $\varphi_{1} \circ \varphi_{2}$ é um isomorfismo normalmente hiperbólico. 
Segue da hiperbolicidade normal que o grupo $\Delta=\left\langle\varphi_{1}, \varphi_{2}\right\rangle$ é não Abeliano. De fato, se $\Delta$ é Abeliano, então $\varphi_{1} \circ \varphi_{2}$ é uma involução e portanto, seus autovalores são todos +1 ou -1. Mas, isto contradiz a definição de hiperbolicidade normal. Além disso, se $\varphi_{1}, \varphi_{2} \neq \mathrm{I},-\mathrm{I}$ temos

(NH1) $1 \leq \operatorname{dim} \mathcal{F}\left(\varphi_{i}\right) \leq n-1, i=1,2$.

(NH2) $\operatorname{dim} \mathcal{F}\left(\varphi_{1} \circ \varphi_{2}\right) \leq n-2$

(NH3) $\mathcal{A}\left(\varphi_{1} \circ \varphi_{2}\right)=\{0\}$.

Onde (NH2) e (NH3) resultam diretamente da hiperbolicidade normal de $\varphi_{1} \circ \varphi_{2}$.

Pelo Corolario 5.4, (NH3) implica que $\operatorname{dim} \mathcal{F}\left(\varphi_{1}\right)=\operatorname{dim} \mathcal{F}\left(\varphi_{2}\right)$.

Os seguintes dois lemas referem-se à classificação de pares de involuções lineares transversais em $\mathbb{R}^{n}, n \geq 2$, sob a condição (NH3).

Lema 5.7: Seja $\varphi_{1}, \varphi_{2}$ um par de involuções lineares transversais em $\mathbb{R}^{n}$ tal que $\mathcal{A}\left(\varphi_{1} \circ\right.$ $\left.\varphi_{2}\right)=\{0\}$ e seja $r=\operatorname{dim} \mathcal{F}\left(\varphi_{1}\right)=\operatorname{dim} \mathcal{F}\left(\varphi_{2}\right)$. Então, $\left(\varphi_{1}, \varphi_{2}\right)$ é linearmente equivalente ao par $\left(\psi_{1}, \psi_{2}\right)$ tal que $\psi_{1}$ e $\psi_{2}$ têm matrizes das seguintes formas:

$$
\psi_{1}=\left(\begin{array}{c|c|c}
-\mathrm{I}_{n-r} & 0 & 0 \\
\hline A_{2} & \mathrm{I}_{n-r} & 0 \\
\hline A_{3} & 0 & \mathrm{I}_{2 r-n}
\end{array}\right), \psi_{2}=\left(\begin{array}{c|c|c}
\mathrm{I}_{n-r} & \mathrm{I}_{n-r} & 0 \\
\hline 0 & -\mathrm{I}_{n-r} & 0 \\
\hline 0 & B_{3} & \mathrm{I}_{2 r-n}
\end{array}\right)
$$

com $A_{2}$ invertível.

Demonstração: Pela Proposição 1.16, $\left(\varphi_{1}, \varphi_{2}\right)$ é linearmente equivalente ao par $\left(\tilde{\psi}_{1}, \tilde{\psi}_{2}\right)$ da forma

$$
\tilde{\psi}_{1}=\left(\begin{array}{c|c|c}
-\mathrm{I}_{n-r} & 0 & 0 \\
\hline \tilde{A}_{2} & \mathrm{I}_{n-r} & 0 \\
\hline \tilde{A}_{3} & 0 & \mathrm{I}_{2 r-n}
\end{array}\right), \tilde{\psi}_{2}=\left(\begin{array}{c|c|c}
\mathrm{I}_{n-r} & \tilde{B}_{1} & 0 \\
\hline 0 & -\mathrm{I}_{n-r} & 0 \\
\hline 0 & \tilde{B}_{3} & \mathrm{I}_{2 r-n}
\end{array}\right) .
$$

Desde que $\mathcal{A}\left(\varphi_{1} \circ \varphi_{2}\right)=\{0\}$, então $\mathcal{A}\left(\tilde{\psi}_{1} \circ \tilde{\psi}_{2}\right)=\{0\}$. Vamos provar que $\tilde{A}_{2}$ é invertível para o qual é suficiente provar que $\operatorname{Ker} \tilde{A}_{2}=\{0\}$. Se $u \in \operatorname{Ker} \tilde{A}_{2} \subset \mathbb{R}^{n-r}$ temos que $\tilde{\psi}_{1} \circ \tilde{\psi}_{2}\left(u, 0,-\frac{1}{2} \tilde{A}_{3}(u)\right)=\left(\begin{array}{c|c|c}-\mathrm{I}_{n-r} & -\tilde{B}_{1} & 0 \\ \hline \tilde{A}_{2} & \tilde{A}_{2} \tilde{B}_{1}-\mathrm{I}_{n-r} & 0 \\ \hline \tilde{A}_{3} & \tilde{A}_{3} \tilde{B}_{1}+\tilde{B}_{3} & \mathrm{I}_{2 r-n}\end{array}\right)\left(\begin{array}{c}u \\ 0 \\ -\frac{1}{2} \tilde{A}_{3}(u)\end{array}\right)=\left(\begin{array}{c}-u \\ 0 \\ \frac{1}{2} \tilde{A}_{3}(u)\end{array}\right)$.

Assim, $\left(u, 0, \frac{1}{2} \tilde{A}_{3}(u)\right) \in \mathcal{A}\left(\psi_{1} \circ \psi_{2}\right)=\{0\}$, e $u=0$. Portanto, $\tilde{A}_{2}$ é invetível.

Analogamente, mostramos que $\tilde{B}_{1}$ é invertível pegando $v \in \operatorname{Ker}\left(\tilde{B}_{1}\right)$ e observando que

$$
\tilde{\psi}_{1} \circ \tilde{\psi}_{2}\left(0, v,-\frac{1}{2} \tilde{B}_{3}(u)\right)=\left(0,-v, \frac{1}{2} \tilde{B}_{3}(u)\right) .
$$


Agora, definimos o isomorfismo linear $H: \mathbb{R}^{n} \rightarrow \mathbb{R}^{n}$ como

$$
H=\left(\begin{array}{c|c|c}
\tilde{B}_{1}^{-1} & 0 & 0 \\
\hline 0 & \mathrm{I}_{n-r} & 0 \\
\hline 0 & 0 & \mathrm{I}_{2 r-n}
\end{array}\right)
$$

e consideremos as involuções $\psi_{1}=H \circ \tilde{\psi}_{1} \circ H^{-1}$ e $\psi_{2}=H \circ \tilde{\psi}_{2} \circ H^{-1}$ as quais são:

$$
\psi_{1}=\left(\begin{array}{c|c|c}
-\mathrm{I}_{n-r} & 0 & 0 \\
\hline A_{2} & \mathrm{I}_{n-r} & 0 \\
\hline A_{3} & 0 & \mathrm{I}_{2 r-n}
\end{array}\right), \psi_{2}=\left(\begin{array}{c|c|c}
\mathrm{I}_{n-r} & \mathrm{I}_{n-r} & 0 \\
\hline 0 & -\mathrm{I}_{n-r} & 0 \\
\hline 0 & B_{3} & \mathrm{I}_{2 r-n}
\end{array}\right) .
$$

Logo, $\left(\varphi_{1}, \varphi_{2}\right)$ é linearmente equivalente a $\left(\psi_{1}, \psi_{2}\right)$.

Como conseqüência direta da Proposição 1.17 temos o seguinte lema:

Lema 5.8: Consideremos os pares de involuções lineares transversais $\left(\psi_{1}, \psi_{2}\right)$ e $\left(\psi_{1}^{\prime}, \psi_{2}^{\prime}\right)$ em $\mathbb{R}^{n}$ com matrizes como em (5.5):

$$
\psi_{1}=\left(\begin{array}{c|c|c}
-\mathrm{I}_{n-r} & 0 & 0 \\
\hline A_{2} & \mathrm{I}_{n-s} & 0 \\
\hline A_{3} & 0 & \mathrm{I}_{r+s-n}
\end{array}\right), \psi_{2}=\left(\begin{array}{c|c|c}
\mathrm{I}_{n-r} & \mathrm{I}_{n-r} & 0 \\
\hline 0 & -\mathrm{I}_{n-r} & 0 \\
\hline 0 & B_{3} & \mathrm{I}_{2 r-n}
\end{array}\right)
$$

$e$

$$
\psi_{1}^{\prime}=\left(\begin{array}{c|c|c}
-\mathrm{I}_{n-r} & 0 & 0 \\
\hline A_{2}^{\prime} & \mathrm{I}_{n-r} & 0 \\
\hline A_{3}^{\prime} & 0 & \mathrm{I}_{2 r-n}
\end{array}\right), \psi_{2}^{\prime}=\left(\begin{array}{c|c|c}
\mathrm{I}_{n-r} & \mathrm{I}_{n-r} & 0 \\
\hline 0 & -\mathrm{I}_{n-r} & 0 \\
\hline 0 & B_{3}^{\prime} & \mathrm{I}_{2 r-n}
\end{array}\right) .
$$

Então $\left(\psi_{1}, \psi_{2}\right)$ e $\left(\psi_{1}^{\prime}, \psi_{2}^{\prime}\right)$ são equivalentes se, e só se, existem matrizes invertíveis $\alpha \in$ $M(n-r)$ e $\beta \in M(2 r-n)$ e as matrizes $\delta, \gamma \in M(2 r-n) \times(n-r)$ tal que

$$
\begin{aligned}
A_{2}^{\prime} & =\alpha A_{2} \alpha^{-1}, \\
A_{3}^{\prime} \alpha & =-2 \delta+\gamma A_{2}+\beta A_{3}, \\
B_{3}^{\prime} \alpha & =\delta B_{1}-2 \gamma+\beta B_{3} .
\end{aligned}
$$

Teorema 5.9: Sejam $\varphi_{1}$ e $\varphi_{2}$ involuções lineares em $\mathbb{R}^{n}$ com $\varphi_{1} \circ \varphi_{2}$ normalmente hiperbólico. Então, $\varphi_{1} \circ \varphi_{2}$ é hiperbólico se, e só se, $n$ é par, $\varphi_{1}$ e $\varphi_{2}$ são transversais e $\operatorname{dim} \mathcal{F}\left(\varphi_{1}\right)=\operatorname{dim} \mathcal{F}\left(\varphi_{2}\right)=n / 2$.

Demonstração: Se $\varphi_{1} \circ \varphi_{2}$ é normalmente hiperbólico, temos que $\operatorname{dim} \mathcal{F}\left(\varphi_{1}\right)=\operatorname{dim} \mathcal{F}\left(\varphi_{2}\right)=$ $r$. Então,

$$
\operatorname{dim} \mathcal{F}\left(\varphi_{1}\right)+\mathcal{F}\left(\varphi_{2}\right)=2 r-\operatorname{dim} \mathcal{F}\left(\varphi_{1}\right) \cap \mathcal{F}\left(\varphi_{2}\right)
$$


Observemos que $\left(\varphi_{1}, \varphi_{2}\right)$ é transversal se, e só se, $\operatorname{dim} \mathcal{F}\left(\varphi_{1}\right) \cap \mathcal{F}\left(\varphi_{2}\right)=2 r-n$. Suponhamos que $\varphi_{1} \circ \varphi_{2}$ é hiperbólico, então $\mathcal{F}\left(\varphi_{1} \circ \varphi_{2}\right)=\{0\}$. Pela Proposição 5.3

$$
\mathcal{F}\left(\varphi_{1}\right) \cap \mathcal{F}\left(\varphi_{2}\right)=\mathcal{A}\left(\varphi_{1}\right) \cap \mathcal{A}\left(\varphi_{2}\right)=\{0\}
$$

Assim, temos

$$
n \geq \operatorname{dim} \mathcal{F}\left(\varphi_{1}\right)+\mathcal{F}\left(\varphi_{2}\right)=\operatorname{dim} \mathcal{F}\left(\varphi_{1}\right)+\operatorname{dim} \mathcal{F}\left(\varphi_{2}\right)-\operatorname{dim} \mathcal{F}\left(\varphi_{1}\right) \cap \mathcal{F}\left(\varphi_{2}\right)=2 r,
$$

o que prova que $n \geq 2 r$. Para provar $2 r \geq n$, vejamos que

$$
n \geq \operatorname{dim} \mathcal{A}\left(\varphi_{1}\right)+\mathcal{A}\left(\varphi_{2}\right)=\operatorname{dim} \mathcal{A}\left(\varphi_{1}\right)+\operatorname{dim} \mathcal{A}\left(\varphi_{2}\right)-\operatorname{dim} \mathcal{A}\left(\varphi_{1}\right) \cap \mathcal{A}\left(\varphi_{2}\right)=2 n-2 r .
$$

Portanto $n=2 r$.

Reciprocamente, suponhamos que $n \geq 2$ é par e $\varphi_{1}$ e $\varphi_{2}$ são transversais com $\operatorname{dim} \mathcal{F}\left(\varphi_{1}\right)=$ $\operatorname{dim} \mathcal{F}\left(\varphi_{2}\right)=n / 2=r$. Pelo Lema 5.7, $\left(\varphi_{1}, \varphi_{2}\right)$ é equivalente a $\left(\phi_{1}, \phi_{2}\right)$, onde

$$
\phi_{1}=\left(\begin{array}{c|c}
-\mathrm{I}_{n-r} & 0 \\
\hline A & \mathrm{I}_{n-r}
\end{array}\right), \quad \phi_{2}=\left(\begin{array}{c|c}
\mathrm{I}_{n-r} & \mathrm{I}_{n-r} \\
\hline 0 & -\mathrm{I}_{n-r}
\end{array}\right),
$$

com $A$ invertível . Agora, pelo Lema 5.8 os pares $\left(\phi_{1}, \phi_{2}\right)$ e $\left(\phi_{1}^{\prime}, \phi_{2}^{\prime}\right)$, onde

$$
\phi_{1}^{\prime}=\left(\begin{array}{c|c}
-\mathrm{I}_{n-r} & 0 \\
\hline A^{\prime} & \mathrm{I}_{n-r}
\end{array}\right), \quad \phi_{2}^{\prime}=\left(\begin{array}{c|c}
\mathrm{I}_{n-r} & \mathrm{I}_{n-r} \\
\hline 0 & -\mathrm{I}_{n-r}
\end{array}\right),
$$

são equivalentes se, e só se, $A$ e $A^{\prime}$ são semelhantes. Portanto, a a partir de agora a matriz $A$ é considerada na sua forma canônica de Jordan.

Agora vejamos que $\operatorname{dim} \mathcal{F}\left(\varphi_{1} \circ \varphi_{2}\right)=\operatorname{dim} \mathcal{F}\left(\phi_{1} \circ \phi_{2}\right)=\operatorname{dim} \operatorname{Ker}\left(\phi_{1} \circ \phi_{2}-\mathrm{I}\right)$, e

$$
\begin{aligned}
\operatorname{dim} \operatorname{Ker}\left(\phi_{1} \circ \phi_{2}-I\right) & =\operatorname{dim} \operatorname{Ker}\left(\begin{array}{c|c}
-2 \mathrm{I}_{r} & -\mathrm{I}_{r} \\
\hline A & A-2 \mathrm{I}_{r}
\end{array}\right) \\
& =\operatorname{dim} \operatorname{Ker}\left(\begin{array}{c|c}
-2 \mathrm{I}_{r} & -\mathrm{I}_{r} \\
\hline 0 & \frac{1}{2} A-2 \mathrm{I}_{r}
\end{array}\right) \\
& =\operatorname{dim} \operatorname{Ker}\left(A-4 \mathrm{I}_{r}\right) .
\end{aligned}
$$

Assim,

$$
\operatorname{dim} \mathcal{F}\left(\varphi_{1} \circ \varphi_{2}\right)=\operatorname{dim} \operatorname{Ker}\left(A-4 \mathrm{I}_{r}\right)
$$

Também vejamos que o polinômio característico de $\varphi_{1} \circ \varphi_{2}$ é

$$
P_{\varphi_{1} \circ \varphi_{2}}(\lambda)=\operatorname{det}\left(\lambda^{2} \mathrm{I}-\lambda\left(A-2 \mathrm{I}_{r}\right)+\mathrm{I}_{r}\right),
$$


pois

$$
\begin{array}{r}
P_{\varphi_{1} \circ \varphi_{2}}(\lambda)=P_{\phi_{1} \circ \phi_{2}}(\lambda)=\operatorname{det}\left(\lambda \mathrm{I}-\phi_{1} \circ \phi_{2}\right)=\operatorname{det}\left(\begin{array}{c|c}
(\lambda+1) \mathrm{I}_{r} & \mathrm{I}_{r} \\
\hline-A & -A+(\lambda+1) \mathrm{I}_{r}
\end{array}\right) \\
=\operatorname{det}\left((\lambda+1)\left(-A+(\lambda+1) \mathrm{I}_{r}\right)+A\right)=\operatorname{det}\left(\lambda^{2} \mathrm{I}-\lambda\left(A-2 I_{r}\right)+\mathrm{I}_{r}\right) .
\end{array}
$$

Para provar que $\varphi_{1} \circ \varphi_{2}$ é hiperbólico, é suficiente provar que $\operatorname{dim} \mathcal{F}\left(\varphi_{1} \circ \varphi_{2}\right)=$ $\operatorname{dim} \operatorname{Ker}\left(A-4 \mathrm{I}_{r}\right)=0$, o qual quer dizer que 4 não é autovalor de $A$. Mas com a hipótese de hiperbolicidade normal não podemos concluir que 4 é autovalor de $A$. Então, suponhamos que 4 é autovalor de $A$ de multiplicidade algébrica $k>0$. Isto é,

$$
A=\left(\begin{array}{c|c}
J_{4}(k) & 0 \\
\hline 0 & B
\end{array}\right),
$$

onde $J_{4}(k)$ é a matriz bloco de Jordan de ordem $k$ do autovalor 4 e $B \in M(r-k)$ é a submatriz de $A$ que contém os autovalores de $A$ diferentes de 4 . Então,

$$
\begin{aligned}
& \operatorname{det}\left(\lambda^{2} \mathrm{I}-\lambda\left(A-2 \mathrm{I}_{r}\right)+\mathrm{I}_{r}\right)= \\
& \left.=\operatorname{det}\left(\begin{array}{c|c}
\lambda^{2} \mathrm{I}_{k} & 0 \\
\hline 0 & \lambda^{2} \mathrm{I}_{r-k}
\end{array}\right)-\lambda\left(\begin{array}{c|c}
J_{2}(k) & 0 \\
\hline 0 & B-2 \mathrm{I}_{r-k}
\end{array}\right)+\left(\begin{array}{c|c}
\mathrm{I}_{k} & 0 \\
\hline 0 & \mathrm{I}_{r-k}
\end{array}\right)\right) \\
& =\operatorname{det}\left(\begin{array}{c|c}
J_{\lambda^{2}-2 \lambda+1}(k) & 0 \\
\hline 0 & \lambda^{2} \mathrm{I}_{r-k}-\lambda\left(B-2 \mathrm{I}_{r-k}\right)+\mathrm{I}_{r-k}
\end{array}\right) .
\end{aligned}
$$

Logo, temos que

$$
P_{\varphi_{1} \circ \varphi_{2}}(\lambda)=\operatorname{det}\left(\lambda^{2} \mathrm{I}-\lambda\left(A-2 \mathrm{I}_{r}\right)+\mathrm{I}_{r}\right)=(\lambda-1)^{2 k} \operatorname{det}\left(\lambda^{2} \mathrm{I}_{r-k}-\lambda\left(B-2 \mathrm{I}_{r-k}\right)+\mathrm{I}_{r-k}\right) .
$$

Assim a multiplicidade algébrica de 1 no polinômio $P_{\varphi_{1} \circ \varphi_{2}}$ é $2 k$, enquanto a multiplicidade geométrica de 1 de $\varphi_{1} \circ \varphi_{2}$ é

$$
\operatorname{dim} \mathcal{F}\left(\varphi_{1} \circ \varphi_{2}\right)=\operatorname{dim} \operatorname{Ker}\left(A-4 \mathrm{I}_{r}\right) \leq k<2 k
$$

o que contradiz a hipótese de hiperboliciade normal, pois para $k>0$ estaríamos concluindo que a multiplicidade geométrica seria diferente da multiplicidade algébrica. Então $\mathcal{F}\left(\varphi_{1} \circ \varphi_{2}\right)=\{0\}$, ou seja, $\varphi_{1} \circ \varphi_{2}$ é hiperbólico.

Corolário 5.10: Sejam $\varphi_{1}$ e $\varphi_{2}$ involuções em $\left(\mathbb{R}^{n}, 0\right)$ com composta normalmente hiperbólica. Então $\varphi_{1} \circ \varphi_{2}$ é hiperbólico se, e só se, $n$ é par $\varphi_{1}$ e $\varphi_{2}$ são transversais e $\operatorname{dim} \mathcal{F}\left(\varphi_{1}\right)=\operatorname{dim} \mathcal{F}\left(\varphi_{2}\right)=n / 2$.

Demonstração: Suponhamos que $\varphi_{1} \circ \varphi_{2}$ é hiperbólico. Então, $d\left(\varphi_{1} \circ \varphi_{2}\right)(0)=d \varphi_{1}(0) \circ$ $d \varphi_{2}(0)$ é hiperbólico onde $d \varphi_{1}(0)$ e $d \varphi_{2}(0)$ são involuções lineares. Pelo teorema anterior, 
$n$ é par, $d \varphi_{1}(0)$ e $d \varphi_{2}(0)$ são transversais, e $\operatorname{dim} \mathcal{F}\left(d \varphi_{1}(0)\right)=\operatorname{dim} \mathcal{F}\left(d \varphi_{2}(0)\right)=n / 2$. Desde que $T_{0} \mathcal{F}\left(\varphi_{i}\right)=\mathcal{F}\left(d \varphi_{i}(0)\right)$, para $i=1,2$, temos que

$$
\operatorname{dim} \mathcal{F}\left(\varphi_{i}\right)=T_{0} \mathcal{F}\left(\varphi_{i}\right)=\operatorname{dim} \mathcal{F}\left(d \varphi_{i}(0)\right)=n / 2, \quad i=1,2
$$

Reciprocamente, suponhamos que $\varphi_{1}$ e $\varphi_{2}$ são involuções transversais e $\operatorname{dim} \mathcal{F}\left(\varphi_{1}\right)=$ $\operatorname{dim} \mathcal{F}\left(\varphi_{2}\right)=n / 2$. Então, $d \varphi_{1}(0)$ e $d \varphi_{2}(0)$ são involuções lineares e

$$
\operatorname{dim} \mathcal{F}\left(d \varphi_{i}(0)\right)=\operatorname{dim} T_{0} \mathcal{F}\left(\varphi_{i}\right)=\operatorname{dim} \mathcal{F}\left(\varphi_{i}\right)=n / 2, \quad i=1,2 .
$$

Finalmente, pelo teorema anterior $d\left(\varphi_{1} \circ \varphi_{2}\right)(0)$ é hiperbólico, o qual prova que $\varphi_{1} \circ \varphi_{2}$ é hiperbólico.

\subsubsection{O caso hiperbólico}

Sejam $\varphi_{1}$ e $\varphi_{2}$ involuções lineares em $\mathbb{R}^{n}$ tais que $\varphi_{1} \circ \varphi_{2}$ é hiperbólico. Pelo Teorema 5.9, $n$ é par, $\varphi_{1}$ e $\varphi_{2}$ são transversais e $\operatorname{dim} \mathcal{F}\left(\varphi_{1}\right)=\operatorname{dim} \mathcal{F}\left(\varphi_{2}\right)=n / 2=r$. Também temos que o par $\left(\varphi_{1}, \varphi_{2}\right)$ é linearmente equivalente ao par $\left(\phi_{1}, \phi_{2}\right)$, onde

$$
\phi_{1}=\left(\begin{array}{c|c}
-\mathrm{I}_{n-r} & 0 \\
\hline A & \mathrm{I}_{n-r}
\end{array}\right), \quad \phi_{2}=\left(\begin{array}{c|c}
\mathrm{I}_{n-r} & \mathrm{I}_{n-r} \\
\hline 0 & -\mathrm{I}_{n-r}
\end{array}\right),
$$

para alguma $A$ invertível. Como já temos visto na prova do Teorema 5.9, $\left(\phi_{1}, \phi_{2}\right)$ é equivalente ao par $\left(\phi_{1}^{\prime}, \phi_{2}^{\prime}\right)$ se, e somente se, $A$ e $A^{\prime}$ são conjugadas . Portanto, no caso hiperbólico a classificação dos pares envolve a classificação de matrizes invertíveis semelhantes de ordem $r$. Então, o estudo deverá proceder considerando as matrizes $A$ na sua forma canônica de Jordan com uma análise onde essas matrizes $A$ levam a composições hiperbólicas.

Assim, isto nos leva a pesquisar o espectro de $A$ de forma que (5.8) não tenha raízes em $S^{1} \subset \mathbb{C}$.

Teorema 5.11: Sejam $\varphi_{1}$ e $\varphi_{2}$ involuções lineares em $\mathbb{R}^{n}$ tal que $\varphi_{1} \circ \varphi_{2}$ é hiperbólico e $r=\operatorname{dim} \mathcal{F}\left(\varphi_{1}\right)=\operatorname{dim} \mathcal{F}\left(\varphi_{2}\right)=n / 2$. Então o par $\left(\varphi_{1}, \varphi_{2}\right)$ é linearmente equivalente ao $\operatorname{par}\left(\phi_{1}, \phi_{2}\right)$, onde

$$
\phi_{1}=\left(\begin{array}{c|c}
-\mathrm{I}_{n-r} & 0 \\
\hline A & \mathrm{I}_{n-r}
\end{array}\right), \quad \phi_{2}=\left(\begin{array}{c|c}
\mathrm{I}_{n-r} & \mathrm{I}_{n-r} \\
\hline 0 & -\mathrm{I}_{n-r}
\end{array}\right),
$$

para alguma matriz invertivel A tal que seus possiveis autovalores reais $\xi$ satisfazem $\xi<0$ ou $\xi>4$, e sem restrições para os autovalores não-reais. 
Demonstração: A primeira parte é conseqüência do Teorema 5.9. Se $\xi$ é um autovalor real de $A$ de multiplicidade geométrica $k$, como na prova do Teorema 5.9 escrevemos

$$
A=\left(\begin{array}{c|c}
J_{\xi}(k) & 0 \\
\hline 0 & B
\end{array}\right),
$$

onde $B \in M(r-k)$ é a submatriz de $A$ que contém os autovalores de $A$ diferentes de $\xi$. Então,

$$
\begin{gathered}
P_{\varphi_{1} \circ \varphi_{2}}(\lambda)=\operatorname{det}\left(\lambda^{2} \mathrm{I}_{r}-\lambda\left(A-2 \mathrm{I}_{r}\right)+\mathrm{I}_{r}\right) \\
=\operatorname{det}\left(\left(\begin{array}{c|c}
\lambda^{2} \mathrm{I}_{k} & 0 \\
\hline 0 & \lambda^{2} \mathrm{I}_{r-k}
\end{array}\right)-\lambda\left(\begin{array}{c|c}
J_{\xi-2}(k) & 0 \\
\hline 0 & B-2 \mathrm{I}_{r-k}
\end{array}\right)+\left(\begin{array}{c|c}
\mathrm{I}_{k} & 0 \\
\hline 0 & \mathrm{I}_{r-k}
\end{array}\right)\right) \\
=\operatorname{det}\left(\begin{array}{c|c}
J_{\lambda^{2}-(\xi-2) \lambda+1}(k) & 0 \\
\hline 0 & \lambda^{2} \mathrm{I}_{r-k}-\lambda\left(B-2 \mathrm{I}_{r-k}\right)+\mathrm{I}_{r-k}
\end{array}\right) \\
=\left(\lambda^{2}-(\xi-2) \lambda+1\right)^{k} \operatorname{det}\left(\lambda^{2} \mathrm{I}_{r-k}-\lambda\left(A-2 \mathrm{I}_{r-k}\right)+\mathrm{I}_{r-k}\right) .
\end{gathered}
$$

Logo, para $\xi \in \mathbb{R}$ a equação $\lambda^{2}-(\xi-2) \lambda+1=0$ tem raízes reais se, e só se, $\xi \leq 0$ ou $\xi \geq 4$. Pela hiperbolicidade de $\varphi_{1} \circ \varphi_{2}$, temos que $\xi$ é necessariamente diferente de 0 e 4 . Para $0<\xi<4$ temos que as raízes

$$
\lambda_{1,2}=\frac{\xi-2 \pm \sqrt{(\xi-2)^{2}-4}}{2}
$$

de $\lambda^{2}-(\xi-2) \lambda+1=0$ têm módulo igual a 1. Portanto $\xi<0$ ou $\xi>4$. Suponhamos agora que $\xi=a+b i$ é um autovalor não real de $A$. Por um cálculo direto temos que

$$
\begin{aligned}
P_{\varphi_{1} \circ \varphi_{2}}(\lambda) & =\operatorname{det}\left(\lambda^{2} \mathrm{I}_{r}-\lambda\left(A-2 \mathrm{I}_{r}\right)+\mathrm{I}_{r}\right) \\
& =\left(\left(\lambda^{2}-(a-2) \lambda+1\right)^{2}+\lambda^{2} b^{2}\right) \operatorname{det}\left(\lambda^{2} \mathrm{I}_{r-2}-\lambda\left(B-2 \mathrm{I}_{r-2}\right)+\mathrm{I}_{r-2}\right) .
\end{aligned}
$$

Observando a equação $\left(\lambda^{2}-(a-2) \lambda+1\right)^{2}+\lambda^{2} b^{2}=0$, queremos provar que para $\xi=a+b i$ não real as raízes desta equação têm módulo diferente de 1. Suponhamos então, que $|\lambda|=1$. Se multiplicamos esta equação por $\bar{\lambda}^{2}$ temos que

$$
\left(|\lambda|^{2} \lambda-(a-2)|\lambda|^{2}+\bar{\lambda}\right)^{2}+|\lambda|^{4} b^{2}=(\lambda-(a-2)+\bar{\lambda})+b^{2}=0
$$

Então, por propriedade dos números reais $b=0$ e $\xi$ é real, o que é uma contradição. Portanto, para qualquer $\xi$ autovalor não real de $A, \varphi_{1} \circ \varphi_{2}$ é hiperbólico.

Agora apresentamos a classificação explícita relativa ao Teorema 5.9 para $n=2,4$. 
- O caso $n=2$. Neste caso temos que $\operatorname{dim} \mathcal{F}\left(\varphi_{1}\right)=\operatorname{dim} \mathcal{F}\left(\varphi_{2}\right)=1$ e pelo Teorema $5.11\left(\varphi_{1}, \varphi_{2}\right)$ é linearmente equivalente ao par $\left(\phi_{1}, \phi_{2}\right)$, onde

$$
\phi_{1}=\left(\begin{array}{cc}
-1 & 0 \\
a & 1
\end{array}\right), \quad \phi_{2}=\left(\begin{array}{cc}
1 & 1 \\
0 & -1
\end{array}\right)
$$

com $a<0$ ou $a>4$. Também temos que $\operatorname{tr}\left(\varphi_{1} \circ \varphi_{2}\right)=\operatorname{tr}\left(\phi_{1} \circ \phi_{2}\right)=a-2$. Portanto, $\operatorname{tr}\left(\varphi_{1} \circ \varphi_{2}\right)>0$ ou $\operatorname{tr}\left(\varphi_{1} \circ \varphi_{2}\right)<4$. É interessante notar que esta forma normal do $\operatorname{par}\left(\varphi_{1}, \varphi_{2}\right)$ pode ser obtida diretamente da classificação que aparece em [10, P. 66], como segue. Primeiro, o grupo $\Delta=\left\langle\varphi_{1}, \varphi_{2}\right\rangle$ gerado por $\varphi_{1}$ e $\varphi_{2}$ é não Abeliano. Logo, desde que $\mathcal{A}\left(\varphi_{1} \circ \varphi_{2}\right)=\{0\}$, então $\mathcal{F}\left(\varphi_{1}\right) \cap \mathcal{A}\left(\varphi_{2}\right)=\{0\}$.

- O caso $n=4$. Aqui temos que $\operatorname{dim} \mathcal{F}\left(\varphi_{1}\right)=\operatorname{dim} \mathcal{F}\left(\varphi_{2}\right)=2$ e pelo Teorema 5.11 $\left(\varphi_{1}, \varphi_{2}\right)$ é linearmente equivalente ao par $\left(\phi_{1}, \phi_{2}\right)$, onde

$$
\phi_{1}=\left(\begin{array}{c|c}
-\mathrm{I}_{2} & 0 \\
\hline A & \mathrm{I}_{2}
\end{array}\right), \quad \phi_{2}=\left(\begin{array}{c|c}
\mathrm{I}_{2} & \mathrm{I}_{2} \\
\hline 0 & -\mathrm{I}_{2}
\end{array}\right),
$$

com $A$ de ordem 2 invertível e na sua forma canônica de Jordan. Sabemos que o polinômio característico de $A$ é

$$
P_{A}(\lambda)=\lambda^{2}-\operatorname{tr}(A) \lambda+\operatorname{det}(A)
$$

Desde que

$$
\phi_{1} \circ \phi_{2}=\left(\begin{array}{c|c}
-\mathrm{I}_{2} & -\mathrm{I}_{2} \\
\hline A & A-\mathrm{I}_{2}
\end{array}\right),
$$

e $\operatorname{tr}\left(\varphi_{1} \circ \varphi_{2}\right)=\operatorname{tr}\left(\phi_{1} \circ \phi_{2}\right)=\operatorname{tr}(A)-4$ e também que

$$
\operatorname{det}\left(\varphi_{1} \circ \varphi_{2}+\mathrm{I}\right)=\operatorname{det}\left(\phi_{1} \circ \phi_{2}+\mathrm{I}\right)=\operatorname{det}\left(\begin{array}{c|c}
0 & -\mathrm{I}_{2} \\
\hline A & A
\end{array}\right)=\operatorname{det}(A)
$$

podemos reescrever o polinômio característico de $A$ da seguinte forma:

$$
P_{A}(\lambda)=\lambda^{2}-\left(4+\operatorname{tr}\left(\varphi_{1} \circ \varphi_{2}\right)\right) \lambda+\operatorname{det}\left(\varphi_{1} \circ \varphi_{2}+\mathrm{I}_{4}\right)
$$

Se $\xi$ é um autovalor real de $A$ com multiplicidade algébrica 2, as possíveis formas de Jordan de $A$ são

$$
\left(\begin{array}{ll}
\xi & \delta \\
0 & \xi
\end{array}\right) \text {, onde } \delta=0 \text { ou } 1
$$

Logo, como

$$
P_{\varphi_{1} \circ \varphi_{2}}(\lambda)=\operatorname{det}\left(\lambda^{2} \mathrm{I}_{r}-\lambda\left(A-2 \mathrm{I}_{r}\right)+\mathrm{I}_{r}\right)
$$


temos que

$$
\begin{aligned}
P_{\varphi_{1} \circ \varphi_{2}}(\lambda) & =\operatorname{det}\left(\left(\begin{array}{cc}
\lambda^{2} & 0 \\
0 & \lambda^{2}
\end{array}\right)-\lambda\left(\begin{array}{cc}
\xi-2 & \delta \\
0 & \xi-2
\end{array}\right)+\left(\begin{array}{ll}
1 & 0 \\
0 & 1
\end{array}\right)\right) \\
& =\operatorname{det}\left(\begin{array}{cc}
\lambda^{2}-(\xi-2) \lambda+1 & \lambda \delta \\
0 & \lambda^{2}-(\xi-2) \lambda+1
\end{array}\right) \\
& =q^{2}(\lambda),
\end{aligned}
$$

$\operatorname{com} q(\lambda)=\lambda^{2}-(\xi-2) \lambda+1$. Suponhamos que $q(\lambda)$ é polinômio minimal de $\varphi_{1} \circ \varphi_{2}$, isto é,

$$
q\left(\varphi_{1} \circ \varphi_{2}\right)=q\left(\phi_{1} \circ \phi_{2}\right)=\left(\begin{array}{cc}
-\left(A-\xi \mathrm{I}_{2}\right) & -\left(A-\xi \mathrm{I}_{2}\right) \\
A\left(A-\xi \mathrm{I}_{2}\right) & \left(A-I_{2}\right)\left(A-\xi \mathrm{I}_{2}\right)
\end{array}\right)=\left(\begin{array}{ll}
0 & 0 \\
0 & 0
\end{array}\right) .
$$

Então, a multiplicidade geométrica de $\xi$ é 2. No caso que $q$ não é polinômio minimal de $\varphi_{1} \circ \varphi_{2}$ temos que a multiplicidade geométrica de $\xi$ é 1 .

\subsubsection{O caso geral}

Consideremos duas involuções lineares $\varphi_{1}$ e $\varphi_{2}$ transversais em $\mathbb{R}^{n}, n \geq 2$, com $\varphi_{1} \circ$ $\varphi_{2}$ normalmente hiperbólico e $\operatorname{dim} \mathcal{F}\left(\varphi_{1}\right)=\operatorname{dim} \mathcal{F}\left(\varphi_{2}\right)=r$. A transversalidade e a hiperbolicidade normal implicam que $n \geq \operatorname{dim} \mathcal{F}\left(\varphi_{1}\right)+\operatorname{dim} \mathcal{F}\left(\varphi_{2}\right)=2 r$ e $r=\operatorname{dim} \mathcal{F}\left(\varphi_{i}\right) \leq$ $n-1$ e portanto,

$$
n / 2 \leq r \leq n-1 .
$$

Mais ainda, pelo Lema 5.7, $\left(\varphi_{1}, \varphi_{2}\right)$ é equivalente a $\left(\psi_{1}, \psi_{2}\right)$ onde $\psi_{1}, \psi_{2}$ têm como matrizes:

$$
\psi_{1}=\left(\begin{array}{c|c|c}
-\mathrm{I}_{n-r} & 0 & 0 \\
\hline A_{2} & \mathrm{I}_{n-r} & 0 \\
\hline A_{3} & 0 & \mathrm{I}_{2 r-n}
\end{array}\right), \psi_{2}=\left(\begin{array}{c|c|c}
\mathrm{I}_{n-r} & \mathrm{I}_{n-r} & 0 \\
\hline 0 & -\mathrm{I}_{n-r} & 0 \\
\hline 0 & B_{3} & \mathrm{I}_{2 r-n}
\end{array}\right)
$$

com $A_{2}$ invertível. Pegando as submatrizes

$$
\phi_{1}=\left(\begin{array}{c|c}
-\mathrm{I}_{n-r} & 0 \\
\hline A_{2} & \mathrm{I}_{n-r}
\end{array}\right), \quad \phi_{2}=\left(\begin{array}{c|c}
\mathrm{I}_{n-r} & \mathrm{I}_{n-r} \\
\hline 0 & -\mathrm{I}_{n-r}
\end{array}\right)
$$

como na prova do Teorema 5.11, temos que

$$
\operatorname{dim} \mathcal{F}\left(\phi_{1} \circ \phi_{2}\right)=\operatorname{Ker}\left(A_{2}-4 I_{n-r}\right) .
$$

Desde que

$$
\begin{aligned}
\operatorname{dim} \mathcal{F}\left(\varphi_{1} \circ \varphi_{2}\right) & =\operatorname{dim} \mathcal{F}\left(\psi_{1} \circ \psi_{2}\right)=\operatorname{dim} \operatorname{Ker}\left(\psi_{1} \circ \psi_{2}-\mathrm{I}\right) \\
& =\operatorname{dim} \operatorname{Ker}\left(\begin{array}{ccc}
-2 \mathrm{I}_{n-r} & -\mathrm{I}_{n-r} & 0 \\
A_{2} & A_{2}-2 \mathrm{I}_{n-r} & 0 \\
A_{3} & A_{3}+B_{3} & 0
\end{array}\right) \\
& \geq 2 r-n .
\end{aligned}
$$


Observemos agora que

$$
\begin{aligned}
\operatorname{dim} \operatorname{Im}\left(\varphi_{1} \circ \varphi_{2}-\mathrm{I}\right) & =\operatorname{dim} \operatorname{Im}\left(\psi_{1} \circ \psi_{2}-\mathrm{I}\right) \\
& =\operatorname{dim} \operatorname{Im}\left(\begin{array}{ccc}
-2 \mathrm{I}_{n-r} & -\mathrm{I}_{n-r} & 0 \\
A_{2} & A_{2}-2 \mathrm{I}_{n-r} & 0 \\
A_{3} & A_{3}+B_{3} & 0
\end{array}\right) \\
& =\operatorname{dim} \operatorname{Im}\left(\begin{array}{cc}
-2 \mathrm{I}_{n-r} & -\mathrm{I}_{n-r} \\
A_{2} & A_{2}-2 \mathrm{I}_{n-r} \\
A_{3} & A_{3}+B_{3}
\end{array}\right) \\
& \geq \operatorname{dim} \operatorname{Im}\left(\begin{array}{cc}
-2 \mathrm{I}_{n-r} & -\mathrm{I}_{n-r} \\
A_{2} & A_{2}-2 \mathrm{I}_{n-r}
\end{array}\right) \\
& =\operatorname{dim} \operatorname{Im}\left(\phi_{1} \circ \phi_{2}-\mathrm{I}_{2 n-2 r}\right),
\end{aligned}
$$

Logo, $\operatorname{dim} \mathcal{F}\left(\varphi_{1} \circ \varphi_{2}\right)=n-\operatorname{dim} \operatorname{Im}\left(\varphi_{1} \circ \varphi_{2}-\mathrm{I}\right) \leq n-\operatorname{Im}\left(\phi_{1} \circ \phi_{2}-\mathrm{I}_{2 n-2 r}\right)=2 r-n+$ $2 n-2 r-\operatorname{Im}\left(\phi_{1} \circ \phi_{2}-\mathrm{I}_{2 n-2 r}\right)=2 r-n+\operatorname{dim} \mathcal{F}\left(\phi_{1} \circ \phi_{2}\right)$. Assim temos que

$$
2 r-n \leq \operatorname{dim} \mathcal{F}\left(\varphi_{1} \circ \varphi_{2}\right) \leq \operatorname{dim} \mathcal{F}\left(\phi_{1} \circ \phi_{2}\right)+2 r-n
$$

O polinômio característico de $\varphi_{1} \circ \varphi_{2}$ é dado por

$$
P_{\varphi_{1} \circ \varphi_{2}}(\lambda)=(\lambda-1)^{2 r-n} P_{\phi_{1} \circ \phi_{2}}(\lambda)=(\lambda-1)^{2 r-n} \operatorname{det}\left(\lambda^{2} \mathrm{I}_{n-r}-\left(A_{2}-2 \mathrm{I}_{n-r}\right) \lambda+\mathrm{I}_{n-r}\right),
$$

pois

$$
\begin{aligned}
P_{\varphi_{1} \circ \varphi_{2}}(\lambda)=\operatorname{det}\left(\lambda \mathrm{I}_{n}-\psi_{1} \circ \psi_{2}\right) & =\operatorname{det}\left(\begin{array}{ccc}
(\lambda+1) \mathrm{I}_{n-r} & -\mathrm{I}_{n-r} & 0 \\
A_{2} & A_{2}+(\lambda+1) \mathrm{I}_{n-r} & 0 \\
A_{3} & -\left(A_{3}+B_{3}\right) & (\lambda-1) \mathrm{I}_{2 r-n}
\end{array}\right) \\
& =(\lambda-1)^{2 r-n} \operatorname{det}\left(\lambda \mathrm{I}_{2 n-2 r}-\phi_{1} \circ \phi_{2}\right) .
\end{aligned}
$$

Agora, a discussão da seção anterior nos leva a concluir que desde que $\varphi_{1} \circ \varphi_{2}$ é normalmente hiperbólico, 4 não é autovalor de $A_{2}$. Mais ainda, dizer que 4 não é autovalor de $A_{2}$ é o mesmo que dizer

$$
\mathcal{F}\left(\phi_{1} \circ \phi_{2}\right)=\{0\}
$$

Então, na desigualdade anterior, temos

$$
\operatorname{dim} \mathcal{F}\left(\varphi_{1} \circ \varphi_{2}\right)=2 r-n
$$

Observemos que com a forma pré-normal $\left(\psi_{1}, \psi_{2}\right)$ do par $\left(\varphi_{1}, \varphi_{2}\right)$ temos a caracterização para a condição de hiperbolicidade normal. Mais precisamente, o fato de que $\varphi_{1} \circ \varphi_{2}$ é normalmente hiperbólico é equivalente a que $\phi_{1} \circ \phi_{2}$ seja hiperbólico. 
Em seguida, é natural perguntar se $A_{3}$ e $B_{3}$ podem ser considerados como matrizes nulas na forma pré-normal. O nosso objetivo no seguinte teorema é provar que de fato podem. Por conseqüência, como mencionado anteriormente, considerando-se a decomposição $\mathbb{R}^{n}=\mathbb{R}^{2(n-r)} \times \mathbb{R}^{2 r-n}$, as formas $\psi_{1}$ e $\psi_{2}$ neste caso são suspensões das formas $\phi_{1} \mathrm{e}$ $\phi_{2}$ em $\mathbb{R}^{2(n-r)}$.

Lembrando a prova da Proposição 2.7 o operador linear $L_{\alpha}: \mathbb{R}^{n-4} \rightarrow \mathbb{R}^{n-4}$ nos dá informação sobre a equivalencia dos pares $\left(\psi_{1}, \psi_{2}\right)$ e $\left(\psi_{1_{b}}, \psi_{2_{b}}\right)$, mais precisamente, $\left(\psi_{1}, \psi_{2}\right)$ e $\left(\psi_{1_{b}}, \psi_{2_{b}}\right)$ são equivalentes se, e só se, $\left(b_{13}, \ldots, b_{2 n}\right) \in \operatorname{Im}\left(T_{v_{\alpha \beta}} \circ L_{\alpha}\right)$ para algum $\beta$ isomorfismo. No seguinte teorema definimos um operador linear similar definido em $M((2 r-n) \times(n-r)) \times M((2 r-n) \times(n-r))$, onde a equivalencia dos pares $\left(\psi_{1}, \psi_{2}\right)$ e $\left(\psi_{1}^{\prime}, \psi_{2}^{\prime}\right)$ na forma $(5.5)$ é valida se, e só se, $A_{2}^{\prime}=\alpha A_{2} \alpha^{-1}$ e $\left(A_{3}, B_{3}\right) \in \operatorname{Im}\left(T_{v_{\alpha \beta}} \circ L_{\alpha}\right)$ para algum $\beta \in M(2 r-n)$ invertível.

Teorema 5.12: Sejam $\varphi_{1}, \varphi_{2}$ involuções lineares em $\mathbb{R}^{n}, n \geq 2$, com $\varphi_{1} \circ \varphi_{2}$ normalmente hiperbólico e $r=\operatorname{dim} \mathcal{F}\left(\varphi_{1}\right)=\operatorname{dim} \mathcal{F}\left(\varphi_{2}\right)$. Então $n / 2 \leq r \leq n-1$ e o par $\left(\varphi_{1}, \varphi_{2}\right)$ é equivalente ao par $\left(\psi_{1}, \psi_{2}\right)$ tal que $\psi_{1}$ e $\psi_{2}$ têm matrizes

$$
\psi_{1}=\left(\begin{array}{c|c|c}
-\mathrm{I}_{n-r} & 0 & 0 \\
\hline A & \mathrm{I}_{n-r} & 0 \\
\hline 0 & 0 & \mathrm{I}_{2 r-n}
\end{array}\right), \psi_{2}=\left(\begin{array}{c|c|c}
\mathrm{I}_{n-r} & \mathrm{I}_{n-r} & 0 \\
\hline 0 & -\mathrm{I}_{n-r} & 0 \\
\hline 0 & 0 & \mathrm{I}_{2 r-n}
\end{array}\right)
$$

com $A$ invertivel e as submatrizes

$$
\phi_{1}=\left(\begin{array}{c|c}
-\mathrm{I}_{n-r} & 0 \\
\hline A & \mathrm{I}_{n-r}
\end{array}\right), \quad \phi_{2}=\left(\begin{array}{c|c}
\mathrm{I}_{n-r} & \mathrm{I}_{n-r} \\
\hline 0 & -\mathrm{I}_{n-r}
\end{array}\right)
$$

nas condições do Teorema 5.11 .

Demonstração: Pelo Lema 1.16, dois pares de involuções lineares transversais $\left(\psi_{1}, \psi_{2}\right)$ e $\left(\psi_{1}^{\prime}, \psi_{2}^{\prime}\right)$ são equivalentes se, e só se

$$
\begin{aligned}
& A_{2}^{\prime}=\alpha A_{2} \alpha^{-1}, \\
& A_{3}^{\prime}=-2 \delta \alpha^{-1}+\gamma A_{2} \alpha^{-1}+\beta A_{3} \alpha^{-1}, \\
& B_{3}^{\prime}=\delta B_{1} \alpha^{-1}-2 \gamma \alpha^{-1}+\beta B_{3} \alpha^{-1},
\end{aligned}
$$

para algumas matrizes $\alpha \in M(n-r)$ e $\beta \in M(2 r-n)$ e $\delta, \gamma \in M(2 r-n) \times(n-r)$ com $\alpha$ e $\beta$ invertíveis. Então, observando estas igualdades, para esta matriz $\alpha$ definimos o operador linear

$L_{\alpha}: M((2 r-n) \times(n-r)) \times M((2 r-n) \times(n-r)) \rightarrow M((2 r-n) \times(n-r)) \times M((2 r-n) \times(n-r))$ 
onde

$$
L_{\alpha}(\delta, \gamma)=\left(\left(-2 \delta+\gamma A_{2}\right) \alpha^{-1},(\delta-2 \gamma) \alpha^{-1}\right)
$$

Para cada $\beta \in M(2 r-n)$ invertível, denotemos $v_{\alpha \beta}$ ao par de matrizes em $M((2 r-n) \times$ $(n-r))$ por

$$
v_{\alpha \beta}=\left(\beta A_{3} \alpha^{-1}, \beta B_{3} \alpha^{-1}\right)
$$

Assim, a equivalência dos pares $\left(\psi_{1}, \psi_{2}\right)$ e $\left(\psi_{1}^{\prime}, \psi_{2}^{\prime}\right)$ é válida se, e só se , existen $\alpha$ e $\beta$ invertíveis tais que

$$
\begin{aligned}
A_{2}^{\prime} & =\alpha A_{2} \alpha^{-1} \\
\left(A_{3}^{\prime}, B_{3}^{\prime}\right) & =\left(\left(-2 \delta+\gamma A_{2}\right) \alpha^{-1},(\delta-2 \gamma) \alpha^{-1}\right)+v_{\alpha \beta}
\end{aligned}
$$

isto é, os pares $\left(\psi_{1}, \psi_{2}\right)$ e $\left(\psi_{1}^{\prime}, \psi_{2}^{\prime}\right)$ são equivalentes se, e só se, $A_{2}^{\prime}=\alpha A_{2} \alpha^{-1}$ e $\left(A_{3}^{\prime}, B_{3}^{\prime}\right) \in$ $\operatorname{Im}\left(T_{v_{\alpha \beta}} \circ L_{\alpha}\right)$.

Afirmação: $L_{\alpha}$ é um isomorfismo. De fato, sejam $(\delta, \gamma),\left(\delta^{\prime}, \gamma^{\prime}\right) \in M((2 r-n) \times(n-$ $r)) \times M((2 r-n) \times(n-r))$ tal que $L_{\alpha}(\delta, \gamma)=L_{\alpha}\left(\delta^{\prime}, \gamma^{\prime}\right)$. Então,

$$
\left(-2 \delta+\gamma A_{2}\right) \alpha^{-1}=\left(-2 \delta^{\prime}+\gamma^{\prime} A_{2}\right) \alpha^{-1}
$$

$\mathrm{e}$

$$
(\delta-2 \gamma) \alpha^{-1}=(\delta-2 \gamma) \alpha^{-1}
$$

Destas últimas equações temos que

$$
\left(\gamma^{\prime}-\gamma\right)\left(A_{2}-4 \mathrm{I}\right)=0
$$

Logo $\gamma^{\prime}=\gamma$, pois 4 não é autovalor de $A_{2}$, com isto na primeria equação também temos que $\delta^{\prime}=\delta$. Portanto, $L_{\alpha}$ é injetora. Para provar que $L_{\alpha}$ é bijetora basta ver que ela pode ser identificada como uma aplicação linear de $\mathbb{R}^{((2 r-n)(n-r))^{2}}$ em $\mathbb{R}^{((2 r-n)(n-r))^{2}}$ injetora. Assim $L_{\alpha}$ é bijetora. Pela sobrejetividade de $T_{v_{\alpha \beta}}$, temos

$$
\operatorname{Im}\left(T_{v_{\alpha \beta}} \circ L_{\alpha}\right)=\operatorname{Im}\left(L_{\alpha}\right)=M((2 r-n) \times(n-r)) \times M((2 r-n) \times(n-r)) .
$$

Vejamos então que $(0,0) \in M((2 r-n) \times(n-r)) \times M((2 r-n) \times(n-r))=\operatorname{Im}\left(T_{v_{\alpha \beta}} \circ L_{\alpha}\right)$, o qual quer dizer que podemos pegar $\left(A_{3}, B_{3}\right)=(0,0)$ e portanto $\left(\psi_{1}, \psi_{2}\right)$ é equivalente a $\left(\psi_{1}^{\prime}, \psi_{2}^{\prime}\right)$, onde

$$
\psi_{1}^{\prime}=\left(\begin{array}{c|c|c}
-\mathrm{I}_{n-r} & 0 & 0 \\
\hline A_{2} & \mathrm{I}_{n-r} & 0 \\
\hline 0 & 0 & \mathrm{I}_{2 r-n}
\end{array}\right), \psi_{2}^{\prime}=\left(\begin{array}{c|c|c}
\mathrm{I}_{n-r} & \mathrm{I}_{n-r} & 0 \\
\hline 0 & -\mathrm{I}_{n-r} & 0 \\
\hline 0 & 0 & \mathrm{I}_{2 r-n}
\end{array}\right)
$$


Finalmente a equivalência de dois pares $\left(\psi_{1}, \psi_{2}\right)$ e $\left(\psi_{1}^{\prime}, \psi_{2}^{\prime}\right)$ na forma normal (5.16) se reduz a dizer que os pares de submatrizes $\left(\phi_{1}, \phi_{2}\right)$ e $\left(\phi_{1}^{\prime}, \phi_{2}^{\prime}\right)$ são equivalentes, onde

$$
\phi_{1}=\left(\begin{array}{c|c}
-\mathrm{I}_{n-r} & 0 \\
\hline A_{2} & \mathrm{I}_{n-r}
\end{array}\right), \quad \phi_{2}=\left(\begin{array}{c|c}
\mathrm{I}_{n-r} & \mathrm{I}_{n-r} \\
\hline 0 & -\mathrm{I}_{n-r}
\end{array}\right)
$$

e

$$
\phi_{1}^{\prime}=\left(\begin{array}{c|c}
-\mathrm{I}_{n-r} & 0 \\
\hline A_{2}^{\prime} & \mathrm{I}_{n-r}
\end{array}\right), \quad \phi_{2}^{\prime}=\left(\begin{array}{c|c}
\mathrm{I}_{n-r} & \mathrm{I}_{n-r} \\
\hline 0 & -\mathrm{I}_{n-r}
\end{array}\right) .
$$

Além disso, as composições $\phi_{1} \circ \phi_{2}$ e $\phi_{1}^{\prime} \circ \phi_{2}^{\prime}$ são hiperbólicas pela hiperbolicidade normal de $\psi_{1} \circ \psi_{2}$ e $\psi_{1}^{\prime} \circ \psi_{2}^{\prime}$ respectivamente. Logo o par $\left(\phi_{1}, \phi_{2}\right)$ esta nas condições do Teorema 5.11 .

Agora aplicamos o Teorema 5.12 para apresentar a classificação explícita de pares de involuções em certas dimensões.

- $n \geq 3$ e $r=n-1$

Primeiro notamos que nestas dimensões a transversalidade é uma propriedade implícita da hiperbolicidade normal. De fato, de (NH2) e de (5.12) temos que

$$
n \geq \operatorname{dim} \mathcal{F}\left(\varphi_{1}\right)+\mathcal{F}\left(\varphi_{2}\right)=2 r-\operatorname{dim} \mathcal{F}\left(\varphi_{1} \circ \varphi_{2}\right) \geq n
$$

Além disso, a forma normal do par $\left(\varphi_{1}, \varphi_{2}\right)$ é dado por

$$
\psi_{1}=\left(\begin{array}{cc|c}
-1 & 0 & 0 \\
a & 1 & 0 \\
\hline 0 & 0 & \mathrm{I}_{n-2}
\end{array}\right), \psi_{2}=\left(\begin{array}{cc|c}
1 & 1 & 0 \\
0 & -1 & 0 \\
\hline 0 & 0 & \mathrm{I}_{n-2}
\end{array}\right)
$$

onde $a<0$ ou $a>4$. Logo temos $\operatorname{tr}\left(\varphi_{1} \circ \varphi_{2}\right)=a+n-4$. Assim, $\operatorname{tr}\left(\varphi_{1} \circ \varphi_{2}\right)>n$ ou $\operatorname{tr}\left(\varphi_{1} \circ \varphi_{2}\right)<n-4$ e $a=4-n+\operatorname{tr}\left(\varphi_{1} \circ \varphi_{2}\right)$.

- $n \geq 3$ e $r=1$

Neste caso as involuções $\varphi_{1}$ e $\varphi_{2}$ não são transversais. Mas, é suficiente observar que

$$
\operatorname{dim} \mathcal{A}\left(\varphi_{i}\right)=\operatorname{dim} \mathcal{F}\left(-\varphi_{i}\right)=n-1 \quad i=1,2 .
$$

Pela Observação 5.6 temos que o par $\left(-\varphi_{1},-\varphi_{2}\right)$ está nas condições do caso anterior. Assim o par $\left(-\varphi_{1},-\varphi_{2}\right)$ é equivalente ao par $\left(\psi_{1}, \psi_{2}\right)$ e portanto, a forma normal do par $\left(\varphi_{1}, \varphi_{2}\right)$ é dado por $\left(-\psi_{1},-\psi_{2}\right)$, com $\left(\varphi_{1}, \varphi_{2}\right)$ como em (5.17). 
- $n \geq 5$ e $r=n-2$

Para estas dimensões a transversalidade do par $\left(\varphi_{1}, \varphi_{2}\right)$ não é sempre válida. Assim para poder apresentar uma classificação neste caso, assumimos esta condição para aplicar o Teorema 5.12. Portanto, a forma normal do par $\left(\varphi_{1}, \varphi_{2}\right)$ é dada por $\left(\psi_{1}, \psi_{2}\right)$, onde

$$
\psi_{1}=\left(\begin{array}{c|c|c}
-\mathrm{I}_{2} & 0 & 0 \\
\hline A & \mathrm{I}_{2} & 0 \\
\hline 0 & 0 & \mathrm{I}_{n-4}
\end{array}\right), \psi_{2}=\left(\begin{array}{c|c|c}
\mathrm{I}_{2} & \mathrm{I}_{2} & 0 \\
\hline 0 & -\mathrm{I}_{2} & 0 \\
\hline 0 & 0 & \mathrm{I}_{n-4}
\end{array}\right)
$$

com $A$ invertível na sua forma canônica de Jordan. Desde que

$$
\psi_{1} \circ \psi_{2}+\mathrm{I}=\left(\begin{array}{c|c|c}
-\mathrm{I}_{2} & -\mathrm{I}_{2} & 0 \\
\hline A & A-\mathrm{I}_{2} & 0 \\
\hline 0 & 0 & \mathrm{I}_{n-4}
\end{array}\right)
$$

temos que

$$
\begin{gathered}
\operatorname{det}\left(\varphi_{1} \circ \varphi_{2}+\mathrm{I}\right)=\operatorname{det}\left(\psi_{1} \circ \psi_{2}+\mathrm{I}\right)=2^{n-4} \operatorname{det}(A) \mathrm{e} \\
\operatorname{tr}\left(\psi_{1} \circ \psi_{2}+\mathrm{I}\right)=n-8+\operatorname{tr}(A) .
\end{gathered}
$$

Também $A$ pode ser tomada na forma do par original $\left(\varphi_{1}, \varphi_{2}\right)$, isto é,

$$
P_{A}(\lambda)=\lambda^{2}-\left(\operatorname{tr}\left(\varphi_{1} \circ \varphi_{2}\right)-n+8\right) \lambda+2^{4-n} \operatorname{det}\left(\varphi_{1} \circ \varphi_{2}+\mathrm{I}_{n}\right)
$$

Como no caso hiperbólico para $n=4$ analisamos a forma canônica de Jordan de A. Se $\xi_{1}$ e $\xi_{2}$ são raízes diferentes, elas podem ser calculadas usando (5.18). Se $A$ tem uma raiz $\xi$ de multiplicidade algébrica 2, a forma canônica de $A$ é

$$
\left(\begin{array}{ll}
\xi & \delta \\
0 & \xi
\end{array}\right) \text {, onde } \delta=0 \text { ou } 1 .
$$

Logo, como

$$
P_{\varphi_{1} \circ \varphi_{2}}(\lambda)=(\lambda-1)^{n-4} \operatorname{det}\left(\lambda^{2} \mathrm{I}_{2}-\lambda\left(A-2 \mathrm{I}_{2}\right)+\mathrm{I}_{2}\right)=(\lambda-1)^{n-4} q^{2}(\lambda)
$$

onde $q(\lambda)=\lambda^{2}-(\xi-2) \lambda+1, q^{2}(\lambda)$ é polinômio característico da submatriz $\phi_{1} \circ$ $\phi_{2}$ hiperbólica de $\varphi_{1} \circ \varphi_{2}$, e $\phi_{1}$ e $\phi_{2}$ são submatrizes de $\varphi_{1}$ e $\varphi_{2}$ respectivamente. Portanto, se $q$ é polinômio minimal de $\phi_{1} \circ \phi_{2}$, $\xi$ tem multiplicidade geométrica 2, e tem multiplicidade geométrica 1 no outro caso. 
Observação 5.13: Para involuções lineares transversais $\varphi_{1}$ e $\varphi_{2}$ tal que a composta é hiperbólica e $\operatorname{dim} \mathcal{F}\left(\varphi_{1}\right)=\operatorname{dim} \mathcal{F}\left(\varphi_{2}\right)=r$, podemos concluir de $\mathcal{F}\left(\varphi_{1}\right) \cap \mathcal{F}\left(\varphi_{2}\right) \subset$ $\mathcal{F}\left(\varphi_{1} \circ \varphi_{2}\right), \operatorname{dim} \mathcal{F}\left(\varphi_{1}\right) \cap \mathcal{F}\left(\varphi_{2}\right)=2 r-n$ e de $(5.14)$ que

$$
\mathcal{F}\left(\varphi_{1} \circ \varphi_{2}\right)=\mathcal{F}\left(\varphi_{1}\right) \cap \mathcal{F}\left(\varphi_{2}\right)
$$

Assim, a Proposição 5.3(a) se reduz a (5.19) com as hipóteses de tranversalidade e hiperbolicidade normal.

Mais ainda, este resultado se generaliza quando $\varphi_{1}$ e $\varphi_{2}$ são involuções transversais em $\left(\mathbb{R}^{n}, 0\right)$ com composta normalmente hiperbólica. Isto é verdade desde que podemos assumir por (5.2) que

$$
\mathcal{F}\left(\varphi_{i}\right)=\mathcal{F}\left(d \varphi_{i}(0)\right) \text {, onde } i=1,2,
$$

$\mathrm{e}$

$$
\mathcal{F}\left(\varphi_{1}\right) \cap \mathcal{F}\left(\varphi_{2}\right)=\mathcal{F}\left(d \varphi_{1}(0)\right) \cap \mathcal{F}\left(d \varphi_{2}(0)\right)=\mathcal{F}\left(d \varphi_{1} \circ \varphi_{2}(0)\right)=T_{0} \mathcal{F}\left(\varphi_{1} \circ \varphi_{2}\right),
$$

onde a última igualdade é conseqüência da hiperbolicidade normal de $\varphi_{1} \circ \varphi_{2}$. Logo, $\mathcal{F}\left(\varphi_{1}\right) \cap \mathcal{F}\left(\varphi_{2}\right)$ e $\mathcal{F}\left(\varphi_{1} \circ \varphi_{2}\right)$ são subvariedades com igual dimensão em $\left(\mathbb{R}^{n}, 0\right)$ tal que $\mathcal{F}\left(\varphi_{1}\right) \cap \mathcal{F}\left(\varphi_{2}\right) \subset \mathcal{F}\left(\varphi_{1} \circ \varphi_{2}\right)$ e, portanto,

$$
\mathcal{F}\left(\varphi_{1} \circ \varphi_{2}\right)=\mathcal{F}\left(\varphi_{1}\right) \cap \mathcal{F}\left(\varphi_{2}\right)
$$




\section{Referências Bibliográficas}

[1] P. Baptispelli,M. Manoel. Some results on reversible-equivariant vector fields. Cadernos de Matemática 6 (2005) 237-263.

[2] P. Baptispelli, M. Manoel. The classification of reversible-equivariant steady-state bifurcations on self-dual spaces. Math. Proc. Camb. Phil. Soc. 145 (2008).

[3] P. Baptispelli. Singularidades e teoria de invariantes em bifurcação reversívelequivariante. Tese de doutorado, ICMC-USP (2007).

[4] H. Cartan. Sur les groupes de transformation analytiques. Actualits Scientifique et Industrielles 198 (1935).

[5] J.P.Dufour. Diagrammes dt'applications différentibles. Thèse, Université des Sciences de Montpellier, France (1979).

[6] A. Garcia, Y. Lequain. Elementos de álgebra. Projeto Euclides, 4a ed., Rio de Janeiro, IMPA (2006).

[7] M. Golubitsky, V. Guillemin. Stable mappings and their singularities. Graduate Texts in Mathematics, Vol. 14. Springer-Verlag, New York-Heidelberg (1973) 209 pp.

[8] R. Hermann. The formal linearization of a semisimple Lie algebra of vector fields about a singular point. Trans. Amer. Math. Soc. 130 (1968) 105-109.

[9] K. Hoffman , R. Kunze. Álgebra Linear. Prencite Hall (1973).

[10] S. Mancini, M. Manoel, M.A. Teixeira. Divergent diagrams of folds and simultaneous conjugacy of involutions. Discrete Contin. Dyn. Syst. 12 (2005), no. 4, 657-674.

[11] S. Mancini, M. Manoel, M.A. Teixeira. Pairs of involutions with normally hyperbolic composition. Bulletin des Sciences Mathématiques. 137 (2012), 418-433. 
[12] D. Montgomery, L. Zippin. Topological transformation groups. Interscience $\mathrm{Pu}-$ blishers, New York-London (1955) 282 pp.

[13] I. Oliveira. Métodos algébricos para a obtenção de formas gerais reversíveisequivariantes. Dissertação de Mestrado, ICMC-USP (2009).

[14] J. Palis. Introdução aos sistemas dinâmicos. Coleção Projeto Euclides (1977).

[15] C. Pugh, S. Michael. Linearization of normally hyperbolic diffeomorphisms and flows. Invent. Math. 10 (1970) $187-198$.

[16] M.A. Teixeira. Generic singularities of discontinuous vector fields. An. Acad. Brasil. Ciênc. 53 (1981), no. 2, 257-260.

[17] M.A. Teixeira. Local and simultaneous structural stability of certain diffeomorphisms. Dynamical systems and turbulence. Lecture Notes in Math. 898 (1981).

[18] M.A. Teixeira. Local reversibility and applications. Real and complex singularities. Res. Notes Math. 412 (2000), 251-265.

[19] M.A. Teixeira. On topological stability of divergent diagrams of folds. Math. Z. 180 (1982), no. 3, 361-371.

[20] Voronin, S. M. Analytic classification of pairs of involutions and its applications. Funktsional. Anal. i Prilozhen. 16 (1982), no. 2, 21-29, 96. 